Agricultural fields and vegetation typical of the Verde Valley, central Arizona. Photo by Brandon T. Forbes.

INSET TOP

A USGS hydrographer measures Verde River discharge, central Arizona. Photo by David W. Anning.

INSET BOTTOM

A ditch diversion in the Verde Valley actively diverting water, June 2010. Photo by Brandon T. Forbes. 


\section{Spatial and Seasonal Variability of Base Flow in the Verde Valley, Central Arizona, 2007 and 2011}

By Bradley D. Garner and Donald J. Bills

Prepared in cooperation with the Verde River Basin Partnership, the Town of Clarkdale, and Yavapai County

Scientific Investigations Report 2012-5192 


\section{U.S. Department of the Interior \\ KEN SALAZAR, Secretary \\ U.S. Geological Survey \\ Marcia K. McNutt, Director}

\section{U.S. Geological Survey, Reston, Virginia: 2012}

This report and any updates to it are available online at: http://pubs.usgs.gov/sir/2012/5192

For more information on the USGS - the Federal source for science about the Earth, its natural and living resources, natural hazards, and the environment, visit http://www.usgs.gov or call 1-888-ASK-USGS.

For an overview of USGS information products, including maps, imagery, and publications,

visit http://www.usgs.gov/pubprod

To order this and other USGS information products, visit http://store.usgs.gov

Any use of trade, product, or firm names is for descriptive purposes only and does not imply endorsement by the U.S. Government.

Although this report is in the public domain, permission must be secured from the individual copyright owners to reproduce any copyrighted materials contained within this report.

Suggested citation:

Garner, B.D., and Bills, D.J., 2012, Spatial and seasonal variability of base flow in the Verde Valley, central Arizona, 2007 and 2011: U.S. Geological Survey Scientific Investigations Report 2012-5192, 33 p. 


\section{Contents}

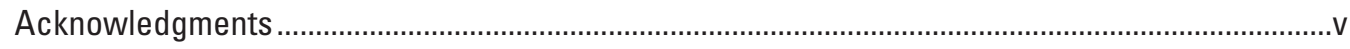

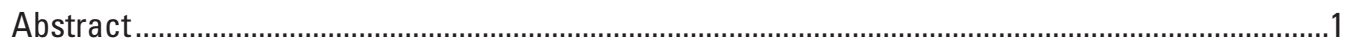

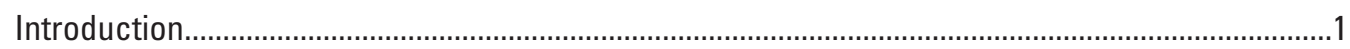

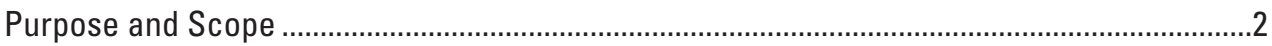

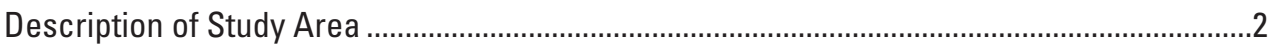

Usage of the Term Base Flow ............................................................................................

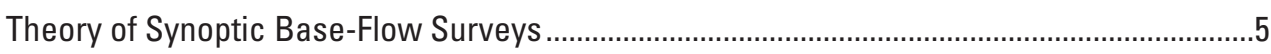

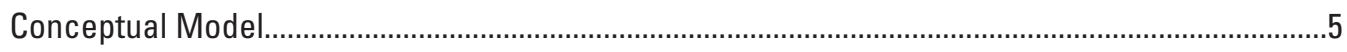

Methods for Data Collection ......................................................................................................

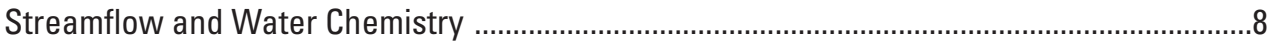

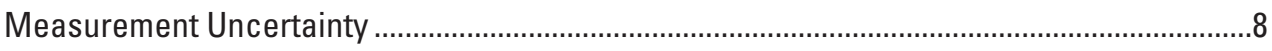

Base Flow in the Verde River, June 2007 and February 2011 .....................................................10

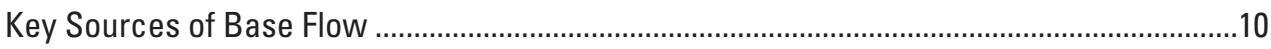

Spatial Variability of Groundwater Fluxes in River Reaches ..................................................19

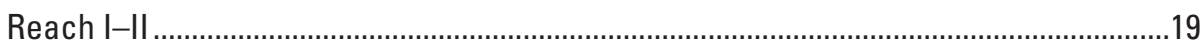

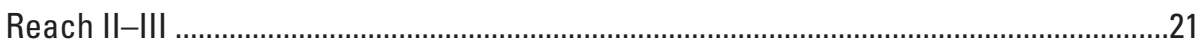

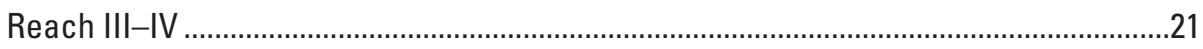

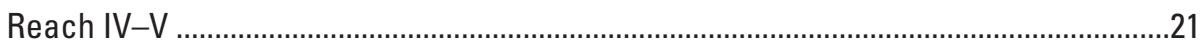

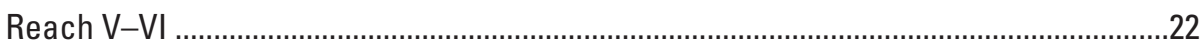

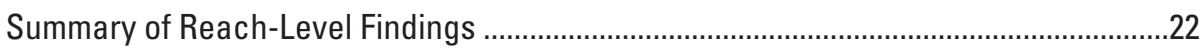

Human Alterations to the Hydrologic System and Their Effects on Base Flow ......................22

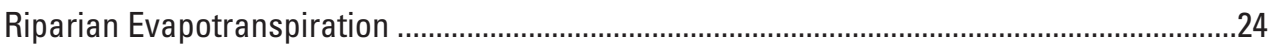

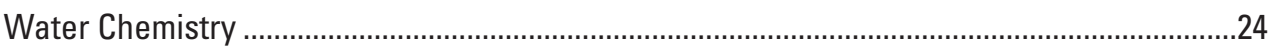

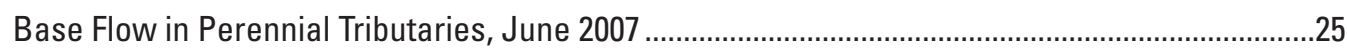

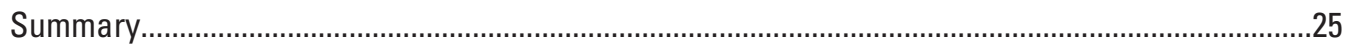

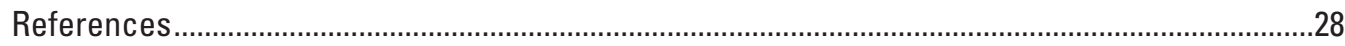

Appendix 1. Derivation of Equations Used in Report...............................................................30

Appendix 2. Compilation of Previously Published Synoptic Base-Flow Data ................................32

\section{Figures}

1. Location of Verde River groundwater basin and the Verde Valley, Arizona................................2

2. Generalized surficial geology of the Verde Valley, central Arizona ............................................

3. Surface-water diversion dam for Tavasci Ditch, Verde Valley, central Arizona..........................4

4. Conceptual diagram of a simple, idealized perennial stream system ...........................................6

5. Conceptual diagram of an idealized perennial stream system with an active irrigation system .......................................................................................................

6. Synoptic base-flow survey measuring stations, 2007 and 2011, Verde Valley, central Arizona.......................................................................................

7. Instantaneous discharge at U.S. Geological Survey streamflow-gaging stations, June-July 2007 and January-February 2011, central Arizona ...........................................10

8. Synoptic base-flow measurements on the Verde River as a function of river mileage, June 2007 and February 2011, Verde Valley, central Arizona. 
9. Oblique aerial photographs of the Verde River, central Arizona

10. Synoptic base-flow measurements of the Verde River, adjusted for surface-water inflows, June 2007 and February 2011, Verde Valley, central Arizona.............................20

11. Instantaneous discharge at the Camp Verde gage, central Arizona, May-June 2006 .........24

12. Schematic diagram of major irrigation ditches along the Verde River as a function of river mileage, Verde Valley, central Arizona.

13. Synoptic base-flow measurements of Oak Creek, June 2007, Verde

Valley, central Arizona. 27

\section{Tables}

1. Synoptic base-flow measurements for the Verde River, tributary confluences with the Verde River, and major ditch diversions, June 2007 and February 2011, Verde Valley, central Arizona.

2. Surface-water and groundwater inflows to the Verde River near confluences with perennial tributaries, June 2007 and February 2011, Verde Valley, central Arizona .......19

3. Measured and calculated flow components for stream reaches along the Verde River, Verde Valley, central Arizona.

4. Water-flow data for major active ditch diversions on the Verde River, June 20-21, 2007, Verde Valley, central Arizona.

5. Water-flow data for major active ditch diversions on the Verde River, February 1-3, 2011, Verde Valley, central Arizona.

6. Synoptic base-flow measurements for Verde River tributaries, June 2007, Verde Valley, central Arizona.

2.1. Synoptic base-flow survey measurements for Verde River mainstem and confluences of tributaries, 1977-1981, Verde Valley, central Arizona.....

\section{Conversion Factors}

Inch/Pound to SI

\begin{tabular}{ccc}
\hline Multiply & By & To obtain \\
\hline & Length & \\
\hline foot $(\mathrm{ft})$ & 0.3048 & meter $(\mathrm{m})$ \\
mile $(\mathrm{mi})$ & 1.609 & kilometer $(\mathrm{km})$ \\
\hline & Volume & \\
\hline acre-foot & 0.001233 & cubic hectometer \\
\hline & Flow rate & \\
\hline cubic foot per second $\left(\mathrm{ft}^{3} / \mathrm{s}\right)$ & 0.02832 & cubic meter per second $\left(\mathrm{m}^{3} / \mathrm{s}\right)$ \\
\hline
\end{tabular}

Temperature in degrees Celsius $\left({ }^{\circ} \mathrm{C}\right)$ may be converted to degrees Fahrenheit $\left({ }^{\circ} \mathrm{F}\right)$ as follows: ${ }^{\circ} \mathrm{F}=\left(1.8 x^{\circ} \mathrm{C}\right)+32$ Vertical coordinate information is referenced to the North American Vertical Datum of 1988. Horizontal coordinate information is referenced to the North American Datum of 1983.

Altitude, as used in this report, refers to distance above the vertical datum. Specific conductance is given in microsiemens per centimeter at 25 degrees Celsius $\left(\mu \mathrm{S} / \mathrm{cm}\right.$ at $\left.25^{\circ} \mathrm{C}\right)$. Concentrations of chemical constituents in water are given in milligrams per liter (mg/L). 


\section{Acknowledgments}

The authors are grateful to the landowners and public entities who gave permission for accessing streams through their property. Several private citizens provided helpful accounts of how rivers, diversions, and ditches have been managed and operated over time. The authors thank the many U.S. Geological Survey staff who collected data under challenging heat and freezing conditions. 



\title{
Spatial and Seasonal Variability of Base Flow in the Verde Valley, Central Arizona, 2007 and 2011
}

\author{
By Bradley D. Garner and Donald J. Bills
}

\section{Abstract}

Synoptic base-flow surveys were conducted on streams in the Verde Valley, central Arizona, in June 2007 and February 2011 by the U.S. Geological Survey (USGS), in cooperation with the Verde River Basin Partnership, the Town of Clarkdale, and Yavapai County. These surveys, also known as seepage runs, measured streamflow under base-flow conditions at many locations over a short period of time. Surveys were conducted on a segment of the Verde River that flows through the Verde Valley, between USGS streamflow-gaging stations 09504000 and 09506000, a distance of 51 river miles. Data from the surveys were used to investigate the dominant controls on Verde River base flow, spatial variability in gaining and losing reaches, and the effects that human alterations have on base flow in the surface-water system. The most prominent human alterations in the Verde Valley are dozens of surface-water diversions from streams, including gravity-fed ditch diversions along the Verde River.

Base flow that entered the Verde River from the tributary streams of Oak Creek, Beaver Creek, and West Clear Creek was found to be a major source of base flow in the Verde River. Groundwater discharge directly into the Verde River near these three confluences also was an important contributor of base flow to the Verde River, particularly near the confluence with Beaver Creek. An examination of individual reaches of the Verde River in the Verde Valley found three reaches (largely unaffected by ditch diversions) exhibiting a similar pattern: a small net groundwater discharge in February 2011 (12 cubic feet per second or less) and a small net streamflow loss in June 2007 (11 cubic feet per second or less). Two reaches heavily affected by ditch diversions were difficult to interpret because of the large number of confounding human factors. Possible lower and upper bounds of net groundwater flux were calculated for all reaches, including those heavily affected by ditches.

\section{Introduction}

The Verde River of central Arizona has perennial (or year-round) flow. In the absence of storm- or snowmelt-related runoff, this perennial flow is sustained by groundwater discharge - a flow component known as base flow. Base flow varies over space and time. Streams may gain base flow from groundwater discharge in some reaches (gaining reaches) and lose base flow in others (losing reaches) where groundwater gradients and streambed characteristics allow surface water to infiltrate into the subsurface. The quantity of water entering or leaving a stream can vary over time in response to short-term and long-term factors. Over time, a stream reach can change from a gaining reach to a losing reach, or from a losing reach to a gaining reach.

Human development of water resources during the 20th century caused many perennial streams in Arizona to become intermittent or ephemeral (Thomas and Pool, 2006; Webb and others, 2007). Presently (2007), Arizona perennial streams such as the San Pedro River are showing decreased base flow, at least in part as a result of human activity (Upper San Pedro Partnership, 2007). This has raised concerns about possible similar base-flow decreases in the Verde River and its associated perennial tributary streams. For centuries, humans and ecosystems have been sustained by base flow in the Verde River and its perennial tributaries (Blasch and others, 2006; Konrad and others, 2008; Ross, 2010; National Park Service, 2012). This report focuses on a portion of the Verde River that flows through the Verde Valley, which is in the middle of the Verde River watershed in central Arizona.

Synoptic base-flow surveys (also known as seepage runs) aid in investigating the groundwater component of streamflow (Harvey and Wagner, 2000; Rosenberry and LaBaugh, 2008, p. 15). Base-flow conditions are ideal times for conducting these surveys, as they minimize some confounding variables. Stormand snowmelt-related runoff components of streamflow can be minimized if a survey is timed correctly. Conducting a survey in the winter months minimizes the effects of vegetation transpiration and diversion of surface water through human infrastructure such as ditches and pumps. Data collected in winter, therefore, are expected to be more indicative of groundwater hydrologic processes; conversely, data collected in summer are expected to reflect additional vegetation and human hydrologic components.

The U.S. Geological Survey (USGS), in cooperation with Yavapai County, Arizona (in 2007) and the Verde River Basin Partnership and the Town of Clarkdale, Arizona (in 
2011), conducted synoptic base-flow surveys on the Verde River in the Verde Valley. Dozens of surface-water diversions in the Verde Valley presented a substantial and ever-present complication for the understanding of base flow. For this and other reasons, one set of surveys was conducted in summer (June 2007) and the other in winter (February 2011). The rationale was that seasonal contrasts could provide insight into the magnitude of effects that diversions have on base flow. Improved understanding of the processes affecting Verde River base flow should enable improved management of the Verde River and its connected groundwater resources.

\section{Purpose and Scope}

The purpose of this report is to publish and describe interpretations of data from synoptic base-flow surveys conducted on the mainstem of the Verde River in the Verde Valley in June 2007 and February 2011, between USGS streamflow-gaging stations 09504000 and 09506000 . Estimates of net groundwater discharge to the Verde River are calculated, although they are uncertain because of measurement uncertainty, human alteration to the hydrologic system, and long-term natural variability. Base-flow data also are published from synoptic base-flow surveys conducted at a coarse spatial scale on perennial tributary streams in the Verde Valley in June 2007.

\section{Description of Study Area}

The study area is the section of the Verde River between USGS streamflow-gaging stations 09504000 (Verde River near Clarkdale, Arizona; hereinafter, the Clarkdale gage) and 09506000 (Verde River near Camp Verde, Arizona; hereinafter, the Camp Verde gage) (fig. 1), as well as sections of three perennial tributary streams: Oak Creek, Beaver Creek, and West Clear Creek (fig. 2). All of the aforementioned stream sections are located in an area of central Arizona known informally as the Verde Valley.

The Verde River flows for 51 river miles $^{1}$ (mi) between the Clarkdale and Camp Verde gages, through the Verde Valley. Along this course it passes over multiple geologic

\footnotetext{
${ }^{1}$ River miles are measured along the course of the river, generally along the thalweg. Because river channels meander and can change after floods, river mileages in this report may not be accurate in the past or the future.
}

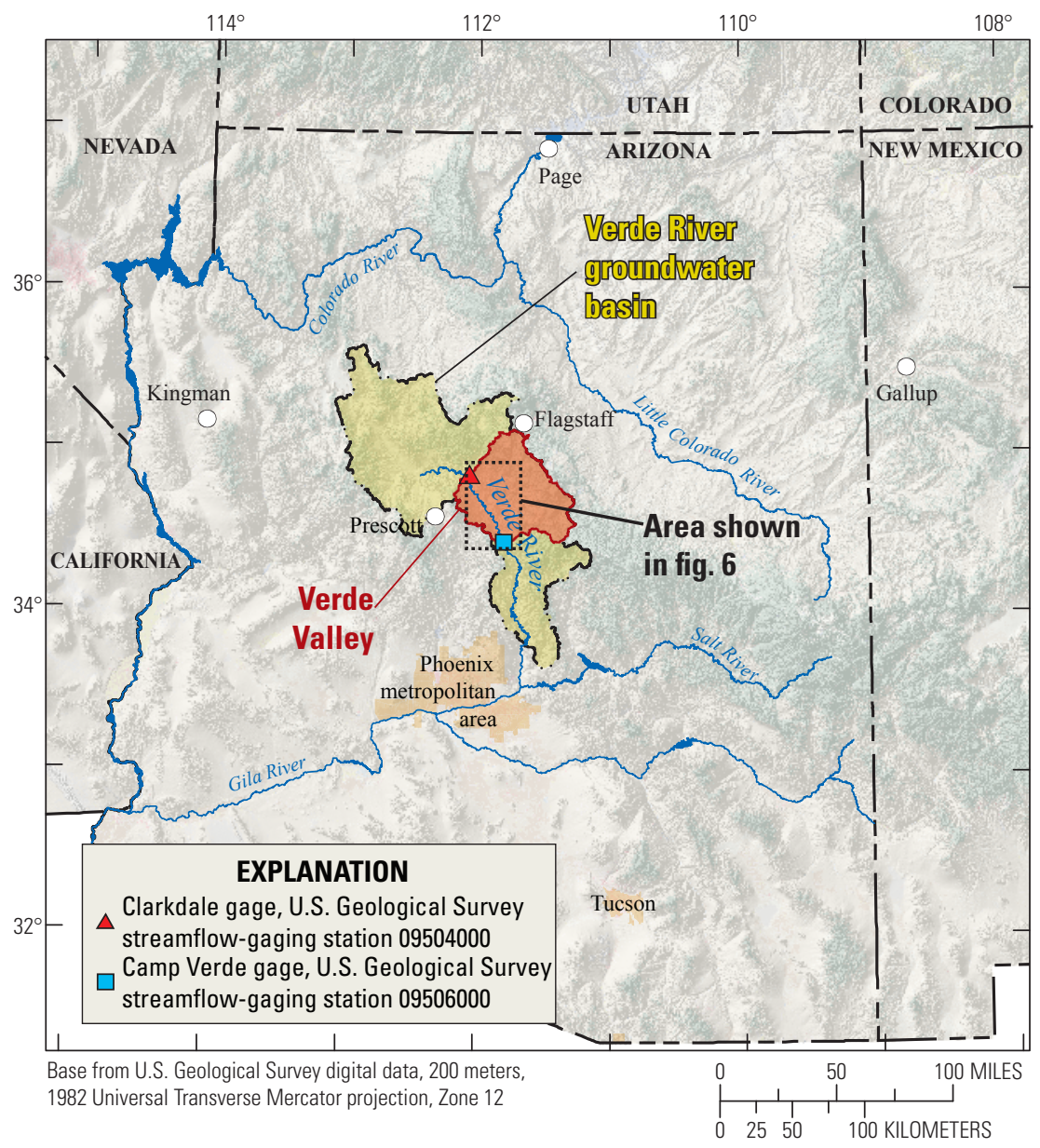

Figure 1. Location of Verde River groundwater basin and the Verde Valley, Arizona. 


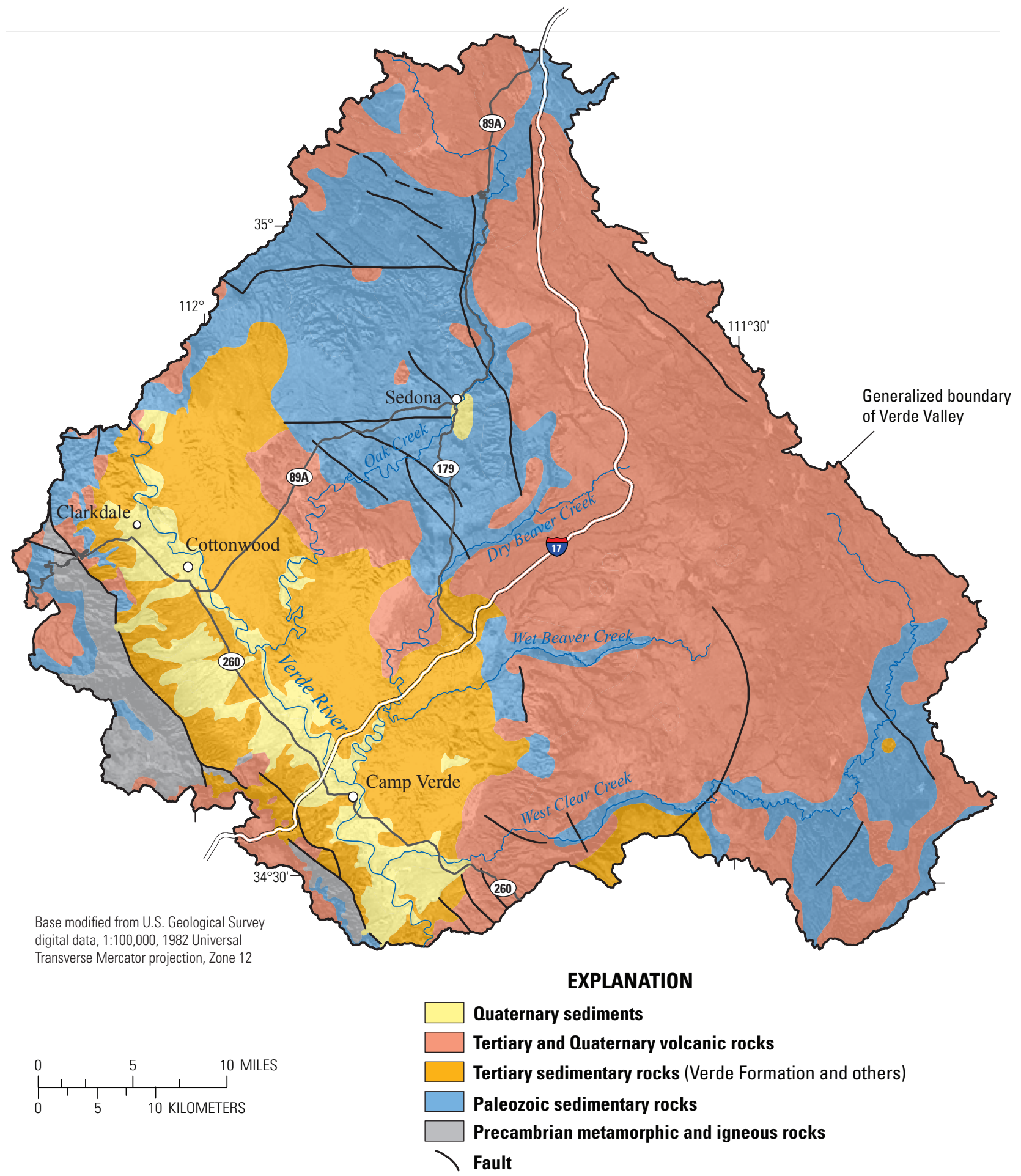

Figure 2. Generalized surficial geology of the Verde Valley, central Arizona (modified from Ludington and others, 2005). 
formations, including Paleozoic sedimentary rocks that contain a regional aquifer, Tertiary volcanic rocks that could be relatively impermeable, Tertiary sedimentary rocks of variable lithology that also contain an aquifer, and thin stringers of Quaternary alluvium associated with the modern stream channel of the Verde River (fig. 2; Blasch and others, 2006; Pool and others, 2011). The Verde River has incised into alluvial fans and Tertiary sedimentary rocks, and these units have been continually reworked into a broad alluvial channel that varies in altitude from 2,900 to 3,500 feet (ft). Geographic distribution and water-bearing characteristics of geologic formations likely affect the distribution of base-flow increases and decreases.

More than 67 river diversions in the Verde Valley deliver surface water to agricultural fields and residential customers (for example, fig. 3). The largest diversions are gravity-fed ditches along the Verde River, some of which divert nearly all available base flow away from the river for one-half of the year or longer (Alam, 1997). Dozens of smaller ditches and pumps (portable and permanent) flank the banks of the Verde River and its perennial tributaries throughout the Verde Valley.

Ditch diversions complicate the ability to investigate and understand natural base-flow processes, because the ditches have altered the hydrology of the Verde Valley considerably. Many ditches have been diverting water for more than 120 years (Alam, 1997), and at least one ditch has been in use for more than a millennium (National Park Service, 2012). Any changes that ditches have imparted to the hydrologic system are challenging to understand, because most ditches were constructed before the first hydrologic investigations in the area.

The ditches diverting water from the Verde River have not been studied comprehensively. Ross (2010) monitored flow rates into and out of four ditches at their headgates and final return flows back to the stream channels; no conclusions

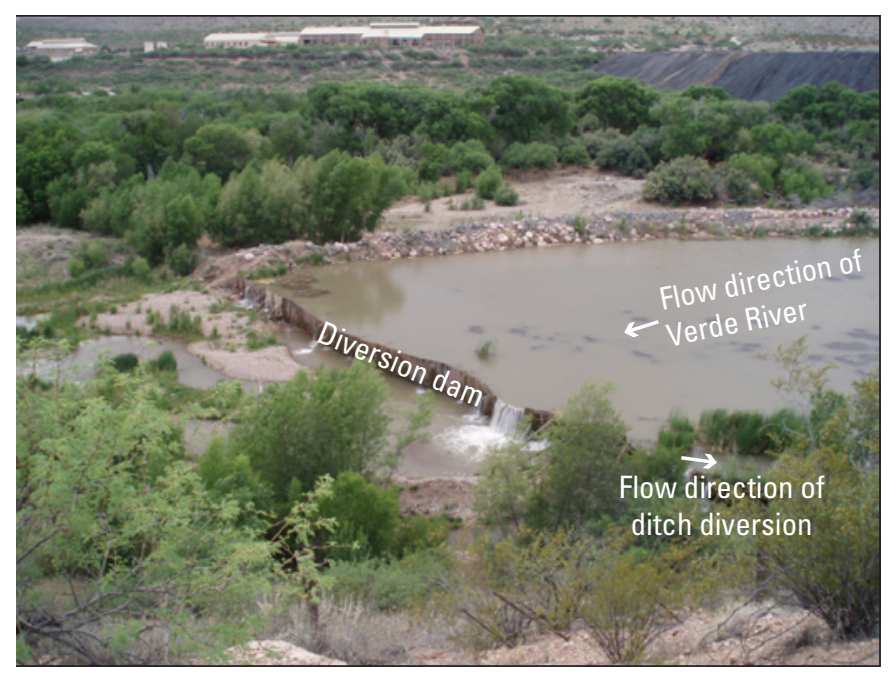

Figure 3. Surface-water diversion dam for Tavasci Ditch, Verde Valley, central Arizona. This dam allows water to divert by gravity flow into Pecks Lake and the Tavasci Ditch. were reached about total water volumes delivered to customers, consumptive-use rates, or the spatial distribution or temporal variably of return flows other than the terminal return flow . Alam (1997) published anecdotal estimates of diverted amounts of water based on surveys of ditch operators. A comprehensive investigation of ditch-diversion hydrology would be possible, but would be a large undertaking well beyond the scope of the present study. Discussion about ditches in this report, therefore, is limited to information that was readily available and measurable.

\section{Usage of the Term Base Flow}

A precise definition and explanation of this report's usage of the term "base flow" is warranted because "an exact definition of base flow varies depending on the author and focus of the study" (Kennedy and Gungle, 2010, p. 5). Base flow "is the portion of streamflow that is derived from persistent, slowly varying sources" (Dingman, 2002, p. 373). In the Verde River watershed, groundwater discharge is the slowly varying source of base flow. However, base flow in the Verde Valley is not necessarily equal to the net discharge of groundwater to streams; such equivalence is possible only in basins with no human alteration of the surface-water system. In the Verde Valley, increases and decreases in base flow can be caused by multiple processes - particularly surface-water ditch diversion.

This report describes streamflow measurements made in the absence of storm- or snowmelt-related runoff ("base-flow conditions") as "base-flow measurements." Such measurements in the Verde Valley may have been altered by human activities, but this usage is consistent with previous reports covering the Verde Valley (Owen-Joyce and Bell, 1983; Owen-Joyce, 1984; Blasch and others, 2006; Pool and others, 2011). Base flow is a term that merits qualification and consideration; therefore the following observations might aid in understanding how the term is used in this report:

- Increases and decreases in base flow are not necessarily equal to net groundwater discharge in a river reach. Other processes, human and natural, may remove or add water to a river reach under base-flow conditions.

- In arid regions, base flow should not be confused with the total amount of groundwater moving toward a stream. A substantial part of groundwater moving toward a stream may be removed by evapotranspiration before it discharges to the stream (Thomas and Pool, 2006).

- So-called summer base-flow, winter base-flow, and annual-average base-flow values all are expected to differ from one another in the Verde Valley, given that some human activities and natural hydrologic processes affect base flow and vary seasonally.

- Even under wholly natural conditions, base flow is not constant, because groundwater gradients change 
in response to varying natural stresses. That is, most hydrologic systems are in dynamic equilibrium, not static equilibrium (Barlow and Leake, in press).

- Base flow can vary not only over long time periods, but also on monthly, weekly, daily, and even hourly time scales. Human activities (for example, diversion of surface water) and natural processes (for example, riparian evapotranspiration) both can cause short-time-scale variations in base flow.

- Base flow in the Verde Valley might be thought of as "potentially diversion-affected base flow," because of the considerable human alterations that have been made to the surface-water system.

\section{Theory of Synoptic Base-Flow Surveys}

A synoptic base-flow survey is like a photograph. The goal is to acquire a snapshot of a moment in time under some ideal condition. Much as photograph exposure time is minimized to avoid blurring, a synoptic base-flow survey is conducted in as short a time as practically possible to avoid changing hydrologic conditions. Much as ideal lighting and weather conditions are awaited before taking a photograph, a synoptic base-flow survey is conducted during base-flow conditions - that is, absence of storm- or snowmelt-related runoff.

Human alterations to a hydrologic system complicate synoptic base-flow surveys. In the Verde Valley, human alteration of the hydrologic system - both direct and indirect - has existed for more than a century.

The primary way humans have affected Verde Valley surface-water systems directly is by diverting surface water into gravity-fed ditches. This is particularly true during the peak summer growing season, but even in the winter months there is some water diversion. Some of the water conveyed through ditches and applied to irrigated fields infiltrates the subsurface. Any of this water that reaches the water table would flow back toward the stream network, where it eventually could discharge as base flow. The timing and extent of such shallow-subsurface return flows have not been studied in the Verde Valley.

Humans can affect surface-water systems indirectly, as well. Withdrawal of groundwater by pumping and incidental recharge can affect gaining and losing stream reaches (Theis, 1940; Leake and Pool, 2010; Leake, 2011). Such stresses affect the groundwater system, and changes to a groundwater system indirectly affect connected surface-water systems. In the Verde River watershed, groundwater and surface-water systems are interconnected (Twenter and Metzger, 1963; Owen-Joyce and Bell, 1983; OwenJoyce, 1984; Blasch and others, 2006; Zlatos, 2008). The effects that groundwater-centric human activities have on connected surface-water systems typically are time-delayed, and are incorporated implicitly into the results of a synoptic base-flow survey. One purpose of repeated synoptic base-flow surveys can be to investigate how human stresses on the groundwater system are altering streamflow in gaining and losing stream reaches over time.

\section{Conceptual Model}

In a simple groundwater basin — "simple" implying absence of human alteration of the surface-water systemsynoptic base-flow surveys can be used to calculate the net exchange (or flux) of groundwater and surface water in a reach of a stream (fig. 4). If streamflow is measured at the upstream and downstream ends of a stream reach, streamflow entering from tributary streams is measured, open-water evaporation is reasonably assumed to be negligible, and steady-state baseflow conditions prevail, then the net exchange of groundwater with the stream is calculated as:

$$
G W_{\text {net }}=Q_{\text {out }}-Q_{\text {in }}-\Sigma Q_{\text {trib }} \pm \varepsilon,
$$

where

$$
\begin{gathered}
G W_{\text {net }} \begin{array}{c}
\text { is net groundwater flux into (positive) or out of } \\
\text { (negative) the mainstem stream reach; }
\end{array} \\
Q_{\text {out }} \text { is mainstem base flow flowing out of the mainstem } \\
\text { stream reach; } \\
Q_{\text {in }} \text { is mainstem base flow flowing into the mainstem } \\
\text { stream reach; } \\
\Sigma Q_{\text {trib }} \text { is the sum of all tributary base flow that flows into } \\
\text { the mainstem stream reach; and } \\
\varepsilon \text { is uncertainty arising from measurement } \\
\text { uncertainty, non-ideal measurement conditions, } \\
\text { and deviations from stated assumptions. }
\end{gathered}
$$

A positive $G W_{n e t}$ value indicates a net discharge of groundwater to the mainstem stream reach - a gaining reach. A negative $G W_{\text {net }}$ value indicates the opposite condition, where there is net infiltration of water in the stream into the subsurface - a losing reach. Values of $G W_{n e t}$ with magnitudes less than $\varepsilon$ are not definitive, but when combined with additional evidence can provide insights into groundwater flux.

Equation (1) is appealing in its simplicity, but does not adequately conceptualize the Verde River in the Verde Valley. Instead, a more complex conceptual model incorporating ditch diversions and irrigation alterations to the system must be used for studying base flow in the Verde Valley (fig. 5). Assuming steady-state conditions in both the stream channels and ditch systems, and negligible open-water evaporation, net groundwater flux $\left(G W_{\text {netND }}\right)$ is calculated as:

$$
\begin{aligned}
& G W_{\text {net ND }}=Q_{\text {out }}-Q_{\text {in }}-\Sigma Q_{\text {trib }}+\Sigma D_{\text {div }}- \\
& \Sigma D_{\text {retMeas }}-\Sigma D_{\text {retUnmeas }}-G W_{\text {inD }} \pm \varepsilon
\end{aligned}
$$

where

$$
\begin{array}{r}
G W_{\text {netND }} \text { is net groundwater flux into (positive) or out } \\
\text { of (negative) the mainstem channel except } \\
\text { for groundwater discharge caused by ditch- } \\
\text { diversion and irrigation systems; } \\
\Sigma D_{d i v} \text { is the sum of all base flow diverted from the } \\
\text { mainstem into the ditch-diversion system; }
\end{array}
$$


$\Sigma D_{\text {retMeas }}$ is the sum of all measured return flows from the ditch-diversion system; ditch return flows exist either because the water was not applied to a field or because it flowed off a field as excess irrigation water ( $F_{\text {out }}$ in fig. 5);

$\Sigma D_{\text {retUnmeas }}$ is the sum of all unmeasured return flows from the ditch-diversion system; and

$G W_{i n D}$ is groundwater discharge into the mainstem channel caused solely by the presence of the ditch-diversion system and irrigation.

Ditch-diversion systems and irrigation practices result in additional water infiltrating the shallow subsurface, which eventually discharges back to the stream $\left(G W_{i n D}\right)$. Some amount of water infiltrates through the bottom of unlined ditches and through agricultural fields ( $I_{d}$ in fig. 5) and becomes part of the groundwater system. The resultant groundwater that discharges to the stream solely because of these human-driven processes is superimposed on the groundwater system that existed before humans altered the surface-water hydrology of the Verde Valley. From an accounting perspective, and as conceived in this conceptual model, it therefore would be incorrect to combine $G W_{\text {inD }}$ with $G W_{\text {net } N D}$.

Values for $\Sigma D_{\text {retUnmeas }}$ and $G W_{i n D}$ are not known, which without additional assumptions would preclude calculation of $G W_{\text {net } N D}$. If the irrigation-system-induced groundwater discharge to the stream is assumed to be negligible $\left(G W_{i n D}=0\right)$ and if unmeasured return flows are assumed to be zero $\left(\Sigma D_{\text {retUnmeas }}=0\right)$, then applying these assumptions to equation (2) results in an upper bound for net groundwater flux ( $\left.G W_{\text {netNDupper }}\right)$ :

$$
G W_{\text {netNDupper }}=Q_{\text {out }}-Q_{\text {in }}-\Sigma Q_{\text {trib }}+\Sigma D_{\text {div }}-\Sigma D_{\text {retMeas }} \pm \varepsilon .
$$

A lower limit $\left(G W_{\text {netNDlower }}\right)$ is calculated by assuming that all diverted water that was not measured as returning to the stream does, in fact, return to the stream $\left(\Sigma D_{\text {retUnmeas }}=\right.$ $\Sigma D_{\text {div }}-\Sigma D_{\text {retMeas }}$ ). Applying this assumption to equation (2) produces:

$$
G W_{\text {netNDlower }}=Q_{\text {out }}-Q_{\text {int }}-\Sigma Q_{\text {trib }} \pm \varepsilon .
$$

If $G W_{i n D}$ is someday determined to be substantially greater than zero, then values of $G W_{\text {netNDlower }}$ and $G W_{\text {netNDupper }}$ in this report will be too large. Therefore, the true value of $G W_{\text {netND }}$ is not necessarily bracketed by the values of $G W_{\text {netNDlower }}$ and $G W_{\text {netNDupper }}$ published in this report. Full derivations of equations (1) through (4), as well as additional discussion about the process of developing them, can be found in appendix 1 .

Additional variables are shown in figure 5, but are not needed in the above equations. The variables are shown only to help the reader conceptualize the ditch-diversion system and to indicate flow components that could be studied in the future to better quantify ditch-diversion systems in the Verde Valley. $D_{\text {ret }}$ is

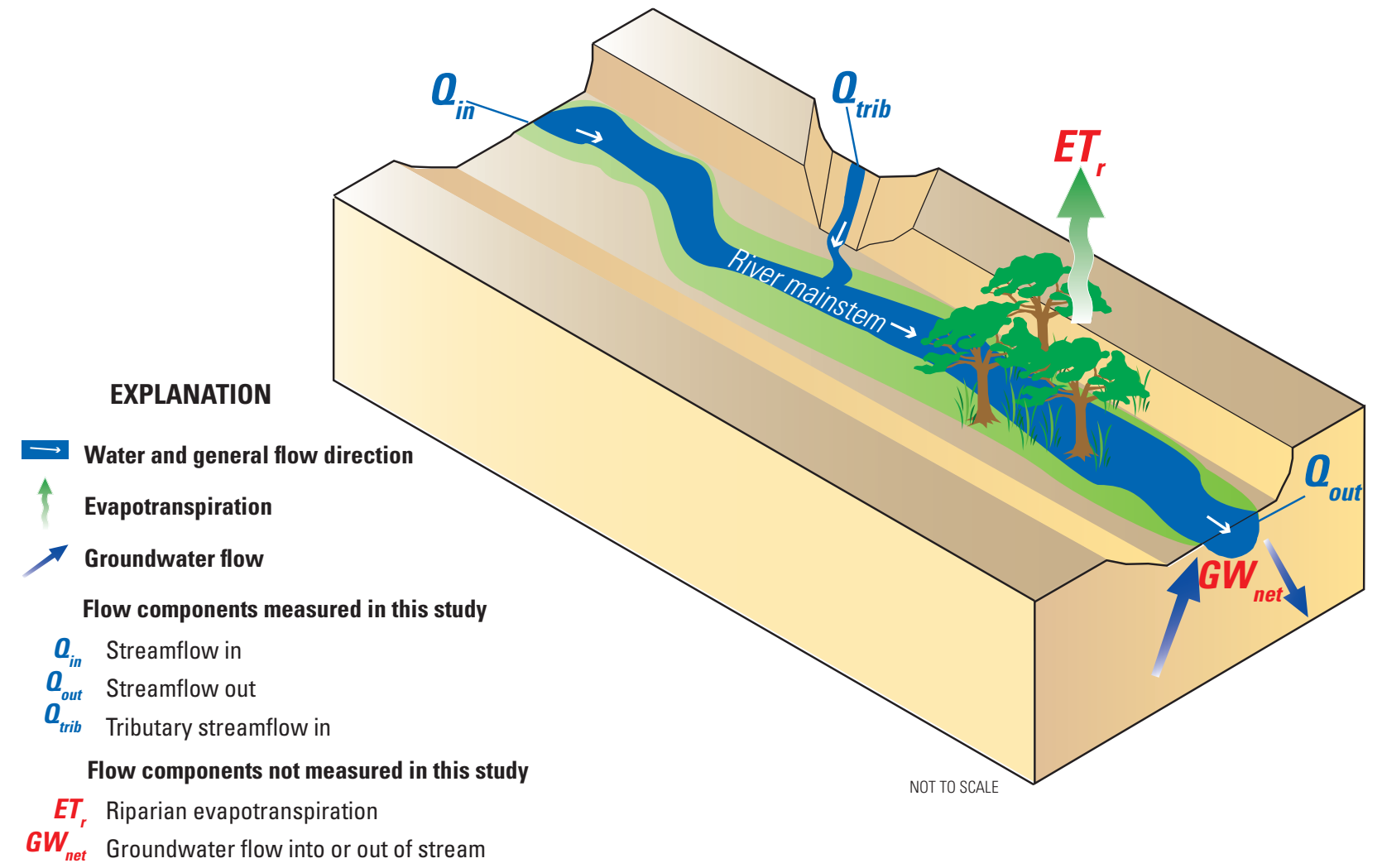

Figure 4. Conceptual diagram of a simple, idealized perennial stream system with no human activity. 


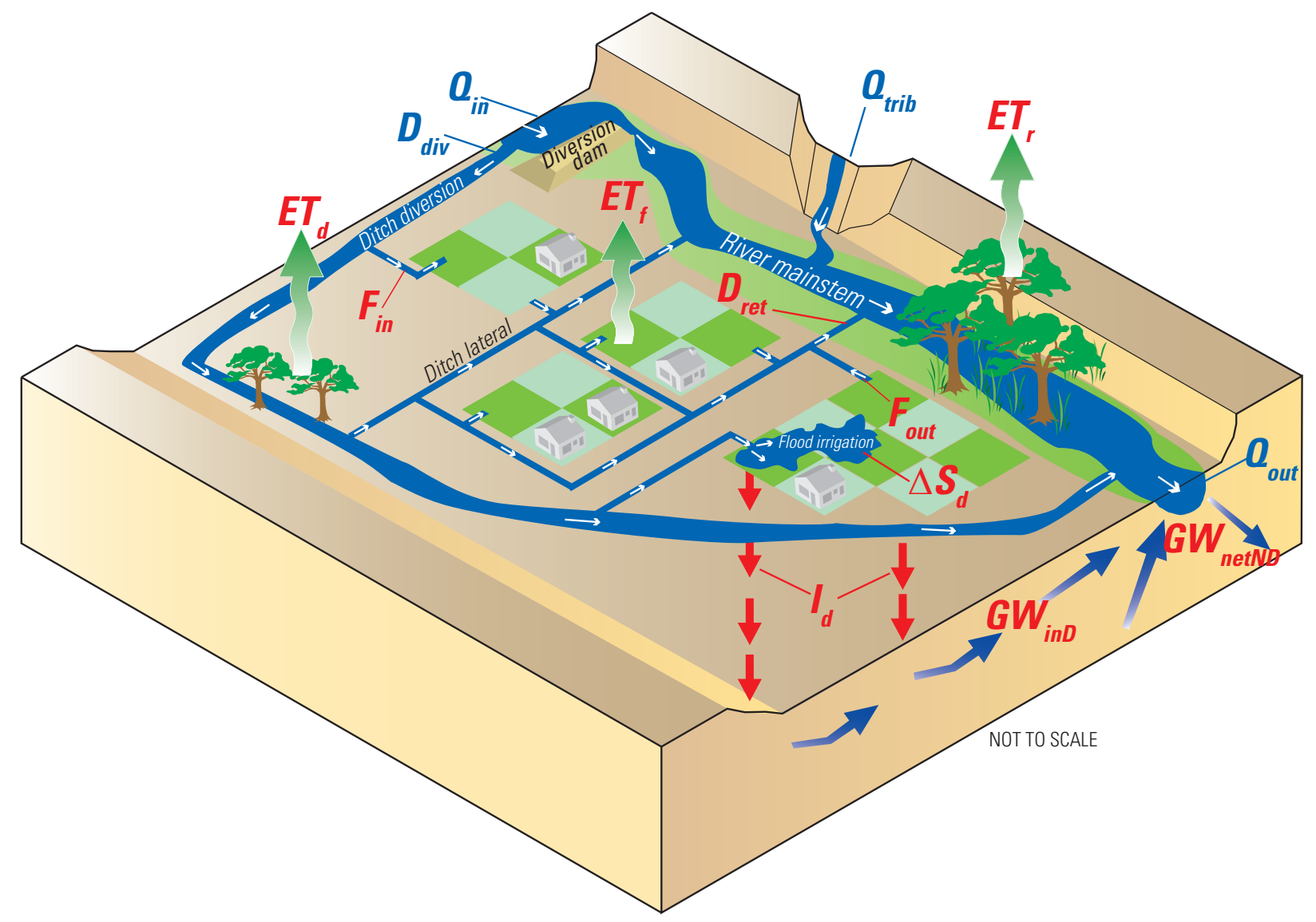

EXPLANATION

$\begin{array}{ll}\text { Water and general flow direction } \\ \text { Evapotranspiration } \\ \text { Groundwater flow } \\ \boldsymbol{Q}_{\text {in }} & \text { Streamflow in } \\ \boldsymbol{Q}_{\text {out }} & \text { Streamflow out } \\ \boldsymbol{Q}_{\text {trib }} & \text { Tributary streamflow in }\end{array}$

Flow components not measured in this study

$E T_{r}$ Riparian evapotranspiration

$E T_{d}$ Evapotranspiration along ditches

$I_{d}$ Infiltration of ditch and irrigation water into subsurface

$\boldsymbol{F}_{i n}$ Irrigation water applied to fields

$E T_{f}$ Evapotranspiration of irrigation water applied to fields

$\Delta \boldsymbol{S}_{d}$ Transient storage change in ditch and irrigation systems

$F_{\text {out }}$ Return flow from fields

$D_{\text {ret }}$ Return flow from ditch-diversion system

$G W_{i n D}$ Discharge to stream of groundwater from ditch infiltration

GW $W_{\text {netND }}$ Groundwater flow into or out of stream, excluding ditch water

Figure 5. Conceptual diagram of an idealized perennial stream system with an active irrigation system (ditch diversions and irrigation). 
a single variable encompassing both measured and unmeasured surface-water return flows (that is, it is equivalent to $D_{\text {retMeas }}$ plus $D_{\text {retUnmeas }}$ ). $E T_{d}$ represents evapotranspiration along ditches, where riparian-like vegetation often grows. $E T_{f}$ represents evapotranspiration from irrigated fields, and is more or less synonymous with "consumptive use" in water-use studies. $F_{\text {in }}$ represents water applied to irrigated fields, and $F_{\text {out }}$ is excess water applied to irrigated fields that flows off those fields and either back into the ditch diversion or into dedicated return-flow canals. $\Delta S_{d}$ represents the fact that, on the scale of hours to weeks, considerably variable volumes of irrigation remain on irrigated fields or are stored in irrigation ponds or stock tanks.

\section{Methods for Data Collection}

Streamflow and selected physicochemical properties were measured in the Verde River and its tributaries in June 2007 and February 2011 using standard USGS methods. Measurement uncertainty was considered when the data were analyzed and interpreted.

\section{Streamflow and Water Chemistry}

Measurements of streamflow (also known as discharge) were made by using the USGS midsection method (Turnipseed and Sauer, 2010). The cross section of a stream channel at a measurement location was divided into subsections, with a goal of less than 5 percent of total streamflow in any one subsection. Velocity in each subsection was measured by using either a mechanical current meter or an Acoustic Doppler Velocity (ADV) meter. The meter was attached to a wading rod that measured water depth and allowed placement of the meter at required depths below the water surface. Velocities were measured at 60 percent of water depth in shallow water; at 20 and 80 percent in deep water; and at 20,60 , and 80 percent if watervelocity profiles were atypical. The threshold between shallow and deep water varied according to the type of velocity meter that was used; for example, the threshold for an ADV was $1.5 \mathrm{ft}$ total water depth. Velocity data were verified and post-processed, and results were entered into the USGS National Water Information System (available at http://waterdata.usgs.gov/).

For each discharge measurement, a corresponding set of physicochemical measurements were made. Measurements of water temperature, specific conductance, dissolved oxygen, and $\mathrm{pH}$ were taken by using instruments from several manufacturers. Sensors were calibrated to reference standards following standard USGS methods (Wilde, variously dated).

Streamflow entering the Verde River from tributary streams was measured in the tributary stream as close as possible to its confluence with the Verde River. These measurements allowed tributary inflows to be subtracted from the results to allow calculation of groundwater flux in the mainstem of the Verde River.
Where water was observed leaving or returning to the Verde River through ditch diversions or their returns flows, attempts were made to measure that streamflow. Because of access restrictions, some diversions had to be calculated indirectly rather than measured directly. This was achieved by subtracting discharge measurements upstream and downstream of the point of interest, which increased measurement uncertainty and required assumptions of no other inflows or outflows of water between the two measurements (see appendix 1). Several small return flows from ditches were measured by using visual estimation through the "float method" (Weight and Sonderegger, 2001, p. 225), resulting in higher uncertainty. Ditch diversions and return flows on perennial tributary streams were not measured.

Three synoptic base-flow surveys were conducted during two time periods (fig. 6). Summertime surveys were conducted on the Verde River June 20-21, 2007, and on perennial tributaries June 26-27, 2007. A wintertime survey was conducted on the Verde River February 1-3, 2011. Measurements were made by four to seven teams of two persons deployed to segments of the stream network each day.

Streamflow records at the Clarkdale gage and Camp Verde gage during all synoptic base-flow surveys were consistent with base-flow conditions (fig. 7). There was no evidence of precipitation, storm-related runoff, or substantial snowmelt-related runoff during any survey. A small amount of flow recession ( 5 cubic feet per second $\left[\mathrm{ft}^{3} / \mathrm{s}\right]$ or less) associated with the end of a snowmelt event occurred during the February 2011 survey.

\section{Measurement Uncertainty}

Quality control, consisting of repeat streamflow measurements, comprised about 10 percent of all measurements. Most quality-control measurements were made by using the midsection method; a few were made by using Acoustic Doppler Current Profile systems that were floated across the stream. Of those made by using the midsection method, most used a velocity-measurement technology that differed from that of the non-quality-control measurement. On the basis of qualitycontrol results, discharge values greater than $10 \mathrm{ft}^{3} / \mathrm{s}$ were rounded to the nearest whole number and values less than $10 \mathrm{ft}^{3} / \mathrm{s}$ were rounded to one decimal place. When considering net groundwater fluxes in a river reach, individual discharge measurements were assumed to have 10-percent uncertainty .

Although 10-percent uncertainty is larger than the uncertainty commonly associated with streamflow measurements made using USGS methods, this higher uncertainty was considered reasonable for data in this study because most of the streamflow measurements were made at river locations that had non-ideal flow conditions and flow-control structures.

There are mathematically and statistically rigorous methods for evaluating discharge-measurement uncertainty (for example, Sauer and Meyer, 1992), but such approaches were beyond the scope of this study. Consideration of 


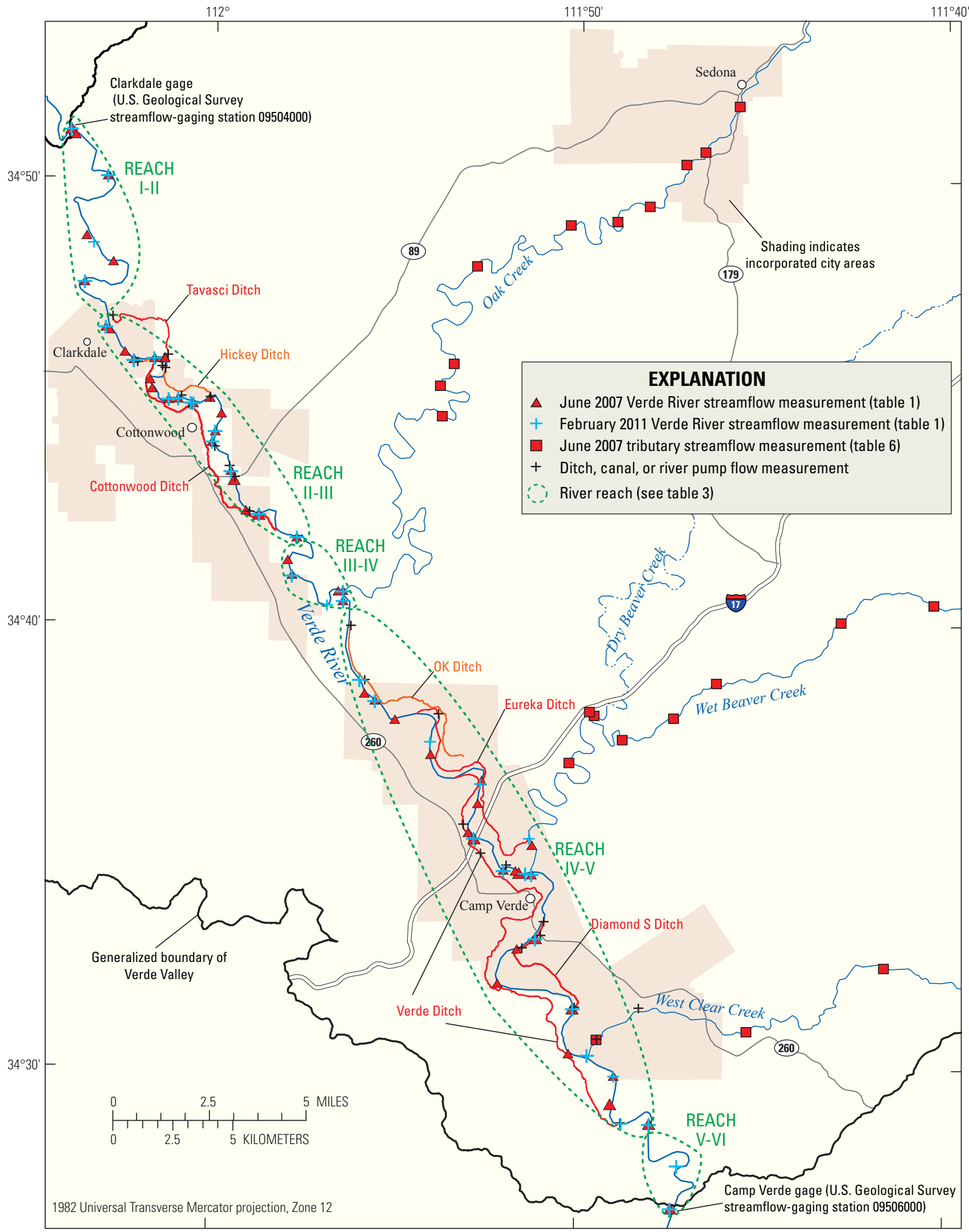

Figure 6. Synoptic base-flow survey measuring stations, 2007 and 2011, Verde Valley, central Arizona. 
uncertainty in this study was largely qualitative; that is, the error term in equations of this report $(\varepsilon)$ was not quantified.

\section{Base Flow in the Verde River, June 2007 and February 2011}

The Verde River presented considerably different flow regimes in June 2007 and February 2011. In February 2011, Verde River flow increased by $140 \mathrm{ft}^{3} / \mathrm{s}$ between the Clarkdale gage and Camp Verde gage. In June 2007 a reverse situation occurred, with an overall flow decrease of $23 \mathrm{ft}^{3} / \mathrm{s}$ between these gages (fig. 8; table 1). Synoptic base-flow survey results and the contrast between winter and summer surveys are discussed in this section. Three challenges for understanding base flow in the Verde Valley are (1) determining the dominant controls on Verde River base flow, (2) determining spatial variability in gaining and losing reaches, and (3) understanding the effects that human alterations have on base flow.

\section{Key Sources of Base Flow}

The perennial tributaries of Oak Creek, Beaver Creek, and West Clear Creek join with the Verde River (figs. 6 and 9 ), and the base flow contributed by these tributaries $\left(\Sigma Q_{\text {trib }}\right.$ on fig. 5) is a major factor in explaining the perennial flow in the Verde River. In February 2011, 84-88 $\mathrm{ft}^{3} / \mathrm{s}$ of tributary surface water entered the Verde River (table 2), with the vast majority $\left(72-76 \mathrm{ft}^{3} / \mathrm{s}\right)$ from Oak Creek. This surface-water contribution of base flow alone accounts for 60 to 63 percent of the observed net streamflow increase between the Clarkdale and Camp Verde gages during the February 2011 base-flow survey. In June 2007, less than one-half as much surface water entered the Verde River from these tributaries $\left(27-33 \mathrm{ft}^{3} / \mathrm{s}\right)$ as in February 2011.

Summertime decreases in base flow in the Verde River and its tributaries have been observed consistently for years (Blasch and others, 2006). This seasonal pattern is caused by seasonally variable human activities (for example, ditch diversion and irrigation) and seasonally variable natural processes (for example, riparian evapotranspiration). Little to no irrigated agriculture occurs in the Verde Valley in the winter (B. Forbes, U.S. Geological Survey, written commun., 2011), and thus few ditches divert water during that time of year. In the summer months, by contrast, irrigation of fields and lawns with water from ditch diversions is common.

Groundwater discharge to the Verde River in the vicinity of the confluences with Oak Creek, Beaver Creek, and West Clear Creek also is an important contributor of base flow to the Verde River. Between 22 and $37 \mathrm{ft}^{3} / \mathrm{s}$ of groundwater discharged to the Verde River near these confluences in February 2011 (table 2), which explains 16 to 26 percent of the total flow gain observed during that synoptic base-flow survey. The quantities of groundwater discharged were less in June $2007\left(18-21 \mathrm{ft}^{3} / \mathrm{s}\right)$ than in February 2011.

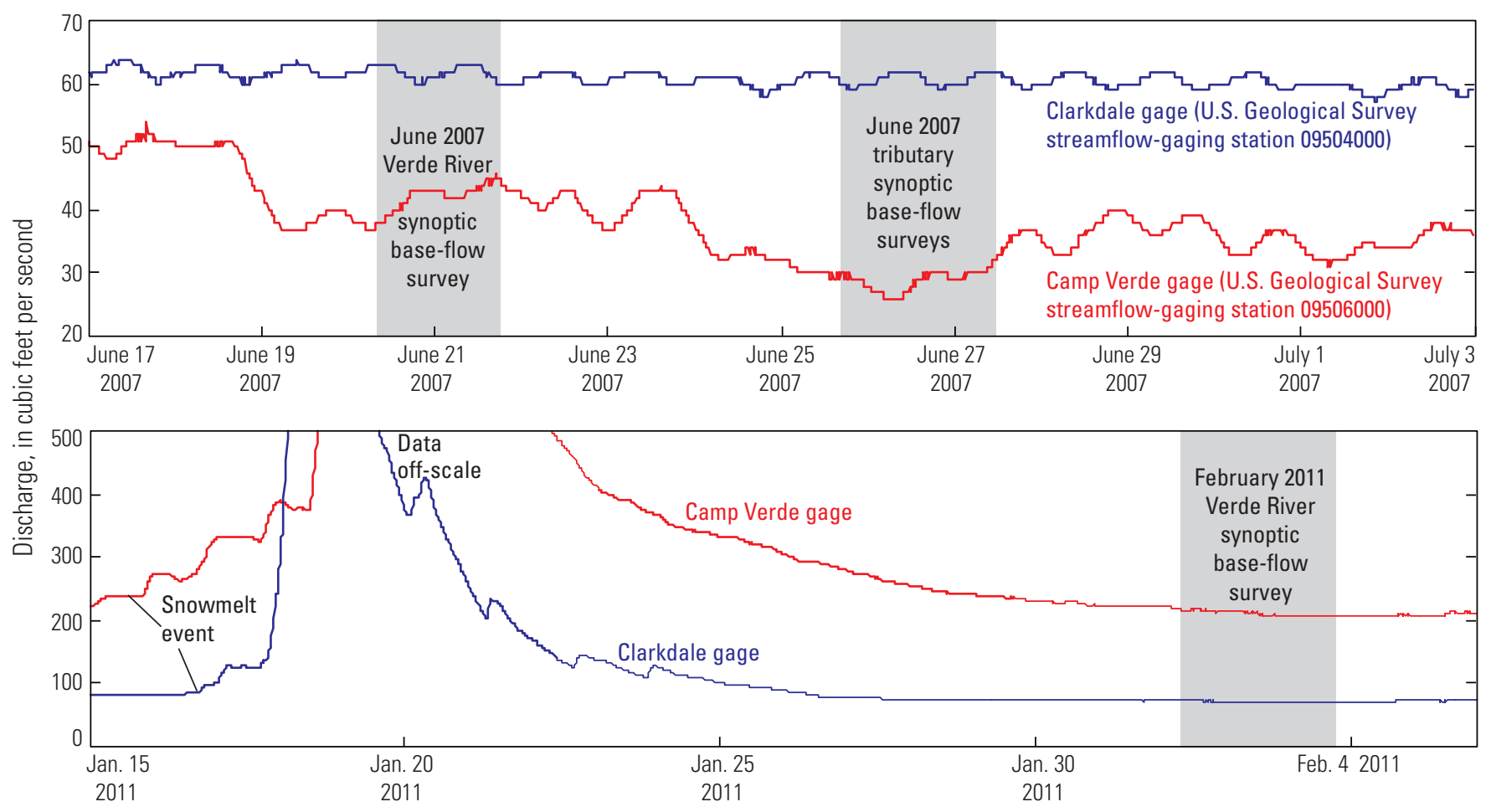

Figure 7. Instantaneous discharge at U.S. Geological Survey streamflow-gaging stations 09504000 and 09506000, June-July 2007 and January-February 2011, central Arizona. 
Verde River synoptic base-flow surveys, 2007 and 2011

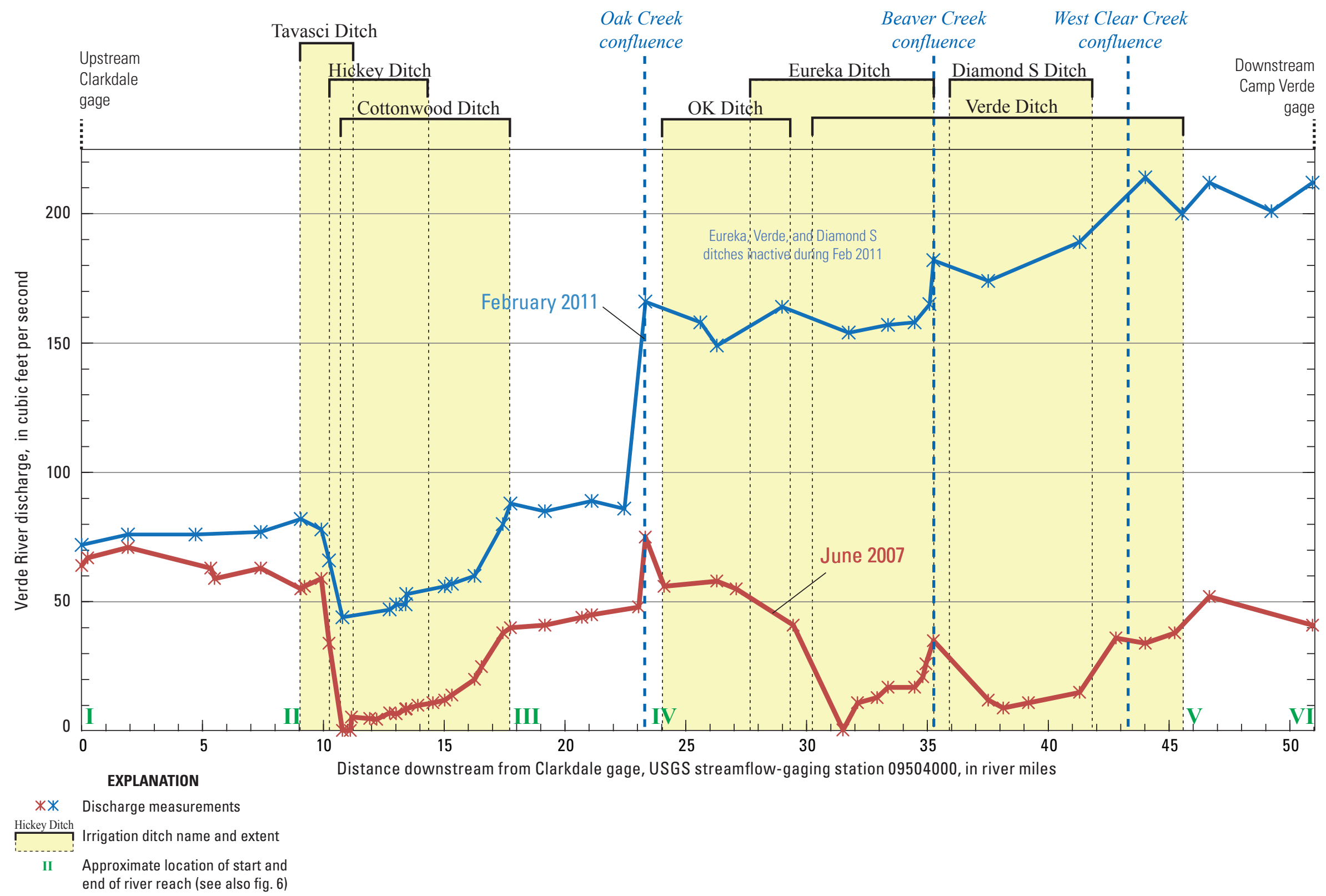

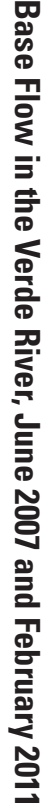

Figure 8. Synoptic base-flow measurements on the Verde River as a function of river mileage, June 2007 and February 2011, Verde Valley, central Arizona. 
Table 1. Synoptic base-flow measurements for the Verde River, tributary confluences with the Verde River, and major ditch diversions, June 2007 and February 2011, Verde Valley, central Arizona.

[Italicized rows indicate measurements not on Verde River mainstem; multiple values indicate repeat measurements for quality assurance; UTM83, Universal Transverse Mercator 1983; ft³, cubic feet per second; cond., conductance; $\mu \mathrm{S} / \mathrm{cm}$, microsiemens per centimeter; ${ }^{\circ} \mathrm{C}$, degrees Celsius; diss., dissolved; $\mathrm{mg} / \mathrm{L}$, milligrams per liter; $\mathrm{pH}$ in standard units; -, no measurement made; >, greater than; $\mathrm{U} / \mathrm{S}$, upstream; $\mathrm{D} / \mathrm{S}$, downstream; R, river]

\begin{tabular}{|c|c|c|c|c|c|c|c|c|c|c|c|c|}
\hline \multirow[b]{2}{*}{ Station identifier } & \multirow[b]{2}{*}{ Station name } & \multirow{2}{*}{$\begin{array}{l}\text { Alternate } \\
\text { station } \\
\text { identifier }\end{array}$} & \multirow{2}{*}{$\begin{array}{l}\text { Latitude } \\
\text { (decimal } \\
\text { degrees, } \\
\text { UTM83) }\end{array}$} & \multirow{2}{*}{$\begin{array}{c}\text { Longitude } \\
\text { (decimal } \\
\text { degrees, } \\
\text { UTM83) }\end{array}$} & \multirow{2}{*}{$\begin{array}{c}\text { River miles } \\
\text { downstream } \\
\text { from station } \\
09504000\end{array}$} & \multicolumn{7}{|c|}{ June 20-21, 2007} \\
\hline & & & & & & Date & Time & $\begin{array}{c}\text { Discharge } \\
\left(\mathrm{ft}^{3} / \mathrm{s}\right)\end{array}$ & $\begin{array}{c}\text { Specific } \\
\text { cond. } \\
(\mu \mathrm{S} / \mathrm{cm})\end{array}$ & $\begin{array}{l}\text { Water } \\
\text { temp. } \\
\left({ }^{\circ} \mathrm{C}\right)\end{array}$ & $\begin{array}{c}\text { Diss. } \\
\text { oxygen } \\
\text { (mg/L) }\end{array}$ & pH \\
\hline 09504000 & VERDE R NEAR CLARKDALE & 1 & 34.852242 & -112.065994 & 0.0 & $6 / 20$ & 9:00 & 64 & 522 & 20.7 & 7.3 & 8.0 \\
\hline 345058112034700 & VERDE R ABOUT 600 FEET BELOW 09504000 & 2 & 34.849464 & -112.063772 & 0.3 & $6 / 20$ & $10: 55$ & 67 & 520 & 21.4 & 7.8 & 8.1 \\
\hline 345004112025400 & VERDE R 1.5 MILES D/S OF GAGE 09504000 & 3 & 34.834464 & -112.049049 & 1.9 & $6 / 20$ & 14:10 & 71 & 506 & 25.1 & 7.7 & 8.3 \\
\hline 344842112032800 & VERDE R ABOVE OLD DUFF DITCH & 4 & 34.811687 & -112.058493 & 5.3 & $6 / 20$ & 17:00 & 63 & 487 & 26.6 & 8.7 & 8.4 \\
\hline 344831112031900 & VERDE R AT REITZ RANCH NEAR CLARKDALE & $4 \mathrm{~A}$ & 34.808722 & -112.055361 & 4.7 & - & - & - & - & - & - & - \\
\hline 344807112024400 & VERDE R BELOW PHELPS DODGE PUMPING STATION & 5 & 34.801965 & -112.046270 & 5.5 & $6 / 20$ & $17: 25$ & $59^{\text {a }} ; 63$ & 484 & 26.7 & 8.4 & 8.4 \\
\hline 344742112033100 & VERDE R 0.5 MILES ABOVE SLAG PILE & 6 & 34.795020 & -112.059326 & 7.4 & $6 / 20$ & 9:00 & $63 ; 63$ & 495 & 22.6 & 7.1 & 8.3 \\
\hline 344652112024500 & PECKS LAKE DIVERSION FROM VERDE RIVER NEAR CLARKDALE & $7 B$ & 34.781132 & -112.046548 & - & - & - & $>0$ & - & - & - & - \\
\hline 344640112025600 & VERDE R BELOW TAVASCI DITCH & 8 & 34.777798 & -112.049603 & 9.1 & $6 / 20$ & $14: 54$ & 55 & 490 & 24.2 & 6.9 & 8.3 \\
\hline 344635112024900 & VERDE R ABOUT 400 FEET D/S OF BITTER CREEK & 9 & 34.776409 & -112.047659 & 9.2 & $6 / 20$ & $15: 30$ & 56 & 490 & 25.4 & 7.8 & 8.3 \\
\hline 344605112022400 & VERDE R 800 FEET U/S OF TUZIGOOT BRIDGE & 10 & 34.768076 & -112.040714 & 9.9 & $6 / 20$ & $8: 10$ & 59 & 494 & 22.5 & 7.2 & 8.3 \\
\hline 344550112020400 & HICKEY DITCH 100 FEET D/S FROM GATE & 11 & 34.763910 & -112.035158 & - & $6 / 20$ & 12:55 & 23 & 494 & 25.6 & 7.6 & 7.6 \\
\hline 344555112020900 & VERDE R BELOW HICKEY DITCH & $12 \mathrm{~A}$ & 34.765215 & -112.036519 & 10.2 & $6 / 20$ & $10: 44$ & 34 & 507 & 23.5 & 6.8 & 8.0 \\
\hline 344554112013500 & COTTONWOOD DITCH BELOW HICKEY DITCH FLUME & 14 & 34.765021 & -112.027102 & - & - & - & $>0$ & - & - & - & - \\
\hline 344558112013600 & VERDE R BELOW COTTONWOOD DITCH & 15 & 34.766215 & -112.027241 & 10.8 & $6 / 20$ & $11: 33$ & $0.3 ; 0.4$ & 550 & 25.5 & 5.7 & 8.0 \\
\hline 344559112011800 & VERDE R ABOVE TAVASCI WASH & 16 & 34.766020 & -112.022435 & 11.1 & $6 / 20$ & 13:09 & 0.3 & 1070 & 29.5 & 6.5 & 7.8 \\
\hline 344600112011700 & TAVASCI MARSH OUTFLOW & $16 A$ & 34.766694 & -112.021306 & - & - & - & - & - & - & - & - \\
\hline 344556112011700 & VERDE R BELOW TAVASCI WASH & 17 & 34.765604 & -112.022463 & 11.2 & $6 / 20$ & 14:00 & 5.5 & 552 & 20.9 & 5.4 & 7.6 \\
\hline 344545112012300 & HICKEY DITCH ABOVE VERDE R FLUME NEAR CLARKDALE & - & 34.762521 & -112.023769 & - & - & - & - & - & - & - & - \\
\hline 344542112011800 & HICKEY DITCH BELOW VERDE R FLUME NEAR CLARKDALE & - & 34.761687 & -112.022380 & - & - & - & - & - & - & - & - \\
\hline 344528112014200 & VERDE R ABOVE MESCAL GULCH & 18 & 34.757771 & -112.029130 & 11.9 & $6 / 20$ & $16: 10$ & 4.9 & 598 & 28.6 & 6.6 & 8.0 \\
\hline 344516112013900 & VERDE R BELOW MESCAL GULCH & 19 & 34.754465 & -112.028158 & 12.2 & $6 / 20$ & $16: 27$ & 4.6 & 621 & 28.2 & 7.1 & 8.0 \\
\hline 344504112010000 & VERDE R BELOW DEADHORSE BRIDGE & 20 & 34.750854 & -112.020713 & 12.8 & $6 / 20$ & $10: 37$ & 7.1 & 685 & 22.6 & 7.7 & 8.0 \\
\hline 344504112005600 & VERDE R ABOVE QUAIL CREEK & 21 & 34.751132 & -112.016268 & 13.0 & $6 / 20$ & $12: 10$ & 6.7 & 681 & 24.9 & 8.3 & 8.0 \\
\hline 344505112005200 & HICKEY DITCH RETURN FLOW NEAR QUAIL CREEK & 22 & 34.751521 & -112.015102 & - & $6 / 20$ & 13:24 & 0.3 & 494 & 26.9 & 8.1 & 8.4 \\
\hline 344458112003300 & VERDE R ABOVE COTTONWOOD DITCH RETURN FLOWS $1 \& 2$ & 23 & 34.749465 & -112.009824 & 13.4 & $6 / 20$ & $11: 42$ & $8.5 ; 9.0$ & 664 & 23.4 & 6.8 & 7.8 \\
\hline 344458112003100 & VERDE R BELOW COTTONWOOD DITCH RETURN FLOWS $1 \& 2$ & 24 & 34.749382 & -112.009296 & 13.4 & $6 / 20$ & $12: 31$ & $8.7 ; 8.8$ & 673 & 23.5 & 7.5 & 7.8 \\
\hline 344504112000300 & VERDE R ABOVE HICKEY DITCH RETURN FLOW & $25 \mathrm{~A}$ & 34.751048 & -112.001712 & 13.9 & $6 / 20$ & $14: 24$ & 10 & 664 & 27.9 & 10.6 & 8.1 \\
\hline 344504112000400 & HICKEY DITCH RETURN FLOW & 26 & 34.751076 & -112.001768 & - & $6 / 20$ & 15:04 & 1.1 & 488 & 25.9 & 7.0 & 8.4 \\
\hline 344443111594500 & VERDE R ABOUT 0.5 MILES U/S OF MINGUS BRIDGE & 27 & 34.745243 & -111.996434 & 14.5 & $6 / 20$ & $17: 16$ & 11 & 623 & 27.7 & 13.3 & 8.2 \\
\hline 344420111595500 & VERDE R BELOW MINGUS BRIDGE & 28 & 34.738910 & -111.999323 & 15.0 & $6 / 20$ & $15: 40$ & $12 ; 12$ & - & - & - & - \\
\hline 344404112000000 & VERDE R ABOVE MOUTH OF SPRING WASH GULCH & 29 & 34.734938 & -112.000795 & 15.3 & $6 / 20$ & $8: 55$ & 14 & 668 & 20.0 & 6.6 & 7.7 \\
\hline 344357111595600 & COTTONWOOD DITCH RETURN FLOW AT GREENWAYLAND & 30 & 34.732438 & -111.999573 & - & $6 / 20$ & 10:20 & 0.7 & 506 & 23.7 & 7.4 & 8.4 \\
\hline 344331111593100 & COTTONWOOD DITCH RETURN FLOW 0.3 MILES U/S OF HIGHWAY $89 \mathrm{~A}$ & $30 \mathrm{~A}$ & 34.725243 & -111.992740 & - & $6 / 20$ & - & $0.8^{b}$ & - & - & - & - \\
\hline 344329111593100 & COTTONWOOD DITCH RETURN FLOW 80 FEET D/S OF SITE $30 A$ & $30 B$ & 34.724854 & -111.992767 & - & $6 / 20$ & - & $2.0^{b}$ & - & - & - & - \\
\hline
\end{tabular}


Table 1. Synoptic base-flow measurements for the Verde River, tributary confluences with the Verde River, and major ditch diversions, June 2007 and February 2011, Verde Valley, central Arizona.-Continued

[Italicized rows indicate measurements not on Verde River mainstem; multiple values indicate repeat measurements for quality assurance; UTM83, Universal Transverse Mercator 1983; ft 3 s, cubic feet per second; cond., conductance; $\mu \mathrm{S} / \mathrm{cm}$, microsiemens per centimeter; ${ }^{\circ} \mathrm{C}$, degrees Celsius; diss., dissolved; $\mathrm{mg} / \mathrm{L}$, milligrams per liter; $\mathrm{pH}$ in standard units; -, no measurement made; >, greater than; $\mathrm{U} / \mathrm{S}$, upstream; $\mathrm{D} / \mathrm{S}$, downstream; R, river]

\begin{tabular}{|c|c|c|c|c|c|c|c|c|c|c|c|c|}
\hline \multirow[b]{2}{*}{ Station identifier } & \multirow[b]{2}{*}{ Station name } & \multirow{2}{*}{$\begin{array}{l}\text { Alternate } \\
\text { station } \\
\text { identifier }\end{array}$} & \multirow{2}{*}{$\begin{array}{l}\text { Latitude } \\
\text { (decimal } \\
\text { degrees, } \\
\text { UTM83) }\end{array}$} & \multirow{2}{*}{$\begin{array}{c}\text { Longitude } \\
\text { (decimal } \\
\text { degrees, } \\
\text { UTM83) }\end{array}$} & \multirow{2}{*}{$\begin{array}{c}\text { River miles } \\
\text { downstream } \\
\text { from station } \\
09504000\end{array}$} & \multicolumn{7}{|c|}{ June 20-21, 2007} \\
\hline & & & & & & Date & Time & $\begin{array}{c}\text { Discharge } \\
\left(\mathrm{ft}^{3} / \mathbf{s}\right)\end{array}$ & $\begin{array}{c}\text { Specific } \\
\text { cond. } \\
\text { ( } \mu \mathrm{S} / \mathrm{cm})\end{array}$ & $\begin{array}{l}\text { Water } \\
\text { temp. } \\
\left({ }^{\circ} \mathrm{C}\right)\end{array}$ & $\begin{array}{c}\text { Diss. } \\
\text { oxygen } \\
\text { (mg/L) }\end{array}$ & $\mathrm{pH}$ \\
\hline 344327111592900 & VERDE R ABOUT 0.25 MILES U/S OF HIGHWAY 89A BRIDGE & 31 & 34.724104 & -111.992017 & 16.3 & $6 / 20$ & $12: 45$ & $20 ; 21$ & 633 & 25.9 & 8.9 & 8.0 \\
\hline 344319111592200 & COTTONWOOD DITCH RETURN FLOW 70 FEET D/S OF HIGHWAY $89 \mathrm{~A}$ & $31 B$ & 34.721069 & -111.990489 & - & $6 / 20$ & 16:07 & 2.5 & 525 & 29.7 & 6.3 & 8.3 \\
\hline 344318111592400 & VERDE R AT HIGHWAY 89A NEAR COTTONWOOD & 32 & 34.720299 & -111.990545 & 16.6 & $6 / 20$ & $16: 00$ & 25 & 626 & 26.2 & 9.0 & 8.0 \\
\hline 344232111590400 & VERDE R 500 FEET ABOVE END OF COTTONWOOD DITCH & 33 & 34.708910 & -111.985156 & 17.4 & $6 / 20$ & $16: 20$ & 38 & 583 & 25.6 & 8.8 & 8.5 \\
\hline 344229111585800 & END OF COTTONWOOD DITCH SITE 34 & 34 & 34.708077 & -111.983489 & - & $6 / 20$ & - & 1.6 & - & - & - & - \\
\hline 344228111584300 & VERDE R BELOW END OF COTTONWOOD DITCH & 35 & 34.708077 & -111.979323 & 17.8 & $6 / 20$ & $16: 30$ & 40 & 578 & 26.1 & 8.6 & 8.5 \\
\hline 344158111574000 & VERDE R AT HEAD OF 2ND BEND BELOW COTTONWOOD DITCH & 36 & 34.699466 & -111.961822 & 19.2 & $6 / 21$ & $14: 40$ & 41 & 623 & 27.4 & 10.6 & 8.3 \\
\hline 344125111575400 & VERDE R AT TAIL OF 2ND BEND ABOVE SPRING & 37 & 34.690383 & -111.965822 & 20.7 & $6 / 21$ & 9:05 & 44 & 593 & 23.2 & 6.1 & 8.2 \\
\hline 344106111574700 & VERDE R AT BLACK MESA DELTA 1.3 MILES U/S OF OAK CREEK & 38 & 34.685022 & -111.963767 & 21.1 & $6 / 21$ & $10: 40$ & 45 & 597 & 23.3 & 5.5 & 8.1 \\
\hline 344026111565000 & VERDE R ABOUT 0.7 MILES U/S OF MOUTH OF OAK CREEK & 38B & 34.673967 & -111.947877 & 22.5 & $6 / 21$ & $12: 15$ & - & 592 & - & 7.5 & 8.3 \\
\hline 344044111563200 & VERDE R ABOVE SPRINGS ABOVE MOUTH OF OAK CREEK & 39 & 34.678855 & -111.942877 & 23.1 & $6 / 21$ & $12: 50$ & 48 & 592 & 25.9 & 6.9 & 8.3 \\
\hline 344045111562400 & OAK CREEK AT VERDE R NEAR CORNVILLE,AZ & $40 B$ & 34.679189 & -111.940710 & - & $6 / 21$ & 13:45 & 30 & 445 & 27.3 & 8.7 & 8.4 \\
\hline 344032111562300 & VERDE R ABOUT 1000 FEET D/S OF MOUTH OF OAK CREEK & 41 & 34.675689 & -111.940572 & 23.3 & $6 / 21$ & $14: 50$ & $75^{\mathrm{c}} ; 65$ & 528 & 27.3 & 7.9 & 8.3 \\
\hline 343956111561100 & OK DITCH 600 FEET D/S FROM THE HEAD & 43 & 34.665578 & -111.937099 & - & $6 / 21$ & 10:57 & 14 & 525 & 24.9 & 5.9 & 8.1 \\
\hline 343843111555500 & VERDE R BELOW OK DITCH TURNOUT NEAR CORNVILLE & 42 & 34.645301 & -111.932654 & 25.6 & $6 / 21$ & $16: 40$ & - & - & - & - & - \\
\hline 343843111554700 & OK DITCH 1.65 MILES BELOW HEADGATE NEAR CAMP VERDE & $43 D$ & 34.645301 & -111.930432 & - & - & - & - & - & - & - & - \\
\hline 343826111554700 & VERDE R U/S OF HAYFIELD DRAW & 44 & 34.640551 & -111.930404 & 24.1 & $6 / 21$ & $13: 12$ & $56 ; 55$ & 506 & 26.5 & 8.9 & 8.3 \\
\hline 343818111553000 & VERDE R D/S OF HAYFIELD DRAW & $44 \mathrm{~A}$ & 34.638440 & -111.925654 & 26.3 & $6 / 21$ & $14: 36$ & 58 & - & 27.7 & - & - \\
\hline 343751111545700 & VERDE R BELOW CHERRY CREEK AND ABOVE EUREKA DITCH & 45 & 34.630884 & -111.916543 & 27.1 & $6 / 21$ & $15: 50$ & 55 & 504 & 28.3 & 10.7 & 8.3 \\
\hline 343758111534600 & EUREKA DITCH AT HEAD NEAR CAMP VERDE & $45 A A$ & 34.632801 & -111.896820 & - & - & - & - & - & - & - & - \\
\hline 343722111535900 & VERDE R D/S OF FORD & $46 \mathrm{~A}$ & 34.622940 & -111.900459 & 29.0 & - & - & - & - & - & - & - \\
\hline 343704111535800 & VERDE R BELOW EUREKA DITCH D/S OF FORD & 47 & 34.617662 & -111.900098 & 29.4 & $6 / 21$ & $9: 47$ & 41 & 530 & 24.5 & 6.4 & 8.1 \\
\hline 343629111523500 & VERDE R BELOW VERDE DITCH & 49 & 34.608163 & -111.877097 & 31.5 & $6 / 21$ & - & $0.5^{\mathrm{d}}$ & 580 & 27.5 & 6.1 & 8.0 \\
\hline 343626111523700 & VERDE R D/S OF VERDE DITCH & $47 \mathrm{~N}$ & 34.607246 & -111.877541 & 31.7 & $6 / 21$ & 13:35 & - & 529 & 27.3 & 7.3 & 8.4 \\
\hline 343558111524000 & VERDE R ABOVE GRIEF HILL WASH & 50 & 34.599468 & -111.878569 & 32.1 & $6 / 21$ & $16: 27$ & 11 & 534 & 28.2 & 9.3 & 8.3 \\
\hline 343529111530400 & VERDE DITCH RETURN FLOW & $50 A$ & 34.591413 & -111.885152 & - & $6 / 21$ & 14:27 & 8 & - & 27.3 & - & - \\
\hline 343519111525500 & VERDE R BELOW GRIEF HILL WASH & 51 & 34.588607 & -111.882763 & 32.9 & $6 / 21$ & $16: 39$ & $13 ; 12$ & 568 & 26.6 & 10.8 & 8.4 \\
\hline 343513111524600 & VERDE R AT I-17 BRIDGE NEAR CAMP VERDE & 52 & 34.586969 & -111.880152 & 33.4 & $6 / 21$ & $9: 58$ & 17 & 638 & 23.6 & 6.6 & 7.9 \\
\hline 343450111523600 & VERDE DITCH RETURN FLOW AT GADDIS WASH NEAR CAMP VERDE & 53 & 34.580580 & -111.877374 & - & $6 / 21$ & 13:00 & 0 & - & - & - & - \\
\hline 343431111515700 & VERDE R ABOVE EUREKA DITCH RETURN FLOW & 54 & 34.575219 & -111.866624 & 34.5 & $6 / 21$ & $11: 55$ & $17 ; 17$ & 756 & 26.2 & 9.2 & 8.1 \\
\hline 343434111515400 & EUREKA DITCH RETURN FLOW BELOW I-17 NEAR CAMP VERDE & $54 \mathrm{~A}$ & 34.576135 & -111.865707 & - & - & - & - & - & - & - & - \\
\hline 343427111513800 & VERDE R ABOVE SPRING AT BLACK BRIDGE & 55 & 34.574191 & -111.861318 & 34.8 & $6 / 21$ & $14: 31$ & 21 & 758 & 27.2 & 12.2 & 8.1 \\
\hline 343424111513300 & VERDE R ABOVE BEAVER CREEK NEAR CAMP VERDE & $55 \mathrm{~A}$ & 34.573358 & -111.859874 & 34.9 & $6 / 21$ & 15:56 & 26 & 795 & 26.1 & 11.5 & 7.9 \\
\hline 343424111512200 & VERDE R 600 FEET ABOVE BEAVER CREEK AT CAMP VERDE & 56 & 34.573358 & -111.856818 & 35.1 & - & - & - & - & - & - & - \\
\hline 343506111511300 & BEAVER CREEK ABOVE EUREKA DITCH NEAR CAMP VERDE & $56 \mathrm{~A}$ & 34.586746 & -111.855235 & - & - & - & - & - & - & - & - \\
\hline
\end{tabular}


Table 1. Synoptic base-flow measurements for the Verde River, tributary confluences with the Verde River, and major ditch diversions, June 2007 and February 2011 , Verde Valley, central Arizona.-Continued

[Italicized rows indicate measurements not on Verde River mainstem; multiple values indicate repeat measurements for quality assurance; UTM83, Universal Transverse Mercator 1983; $\mathrm{ft}^{3} / \mathrm{s}$, cubic feet per second; cond., conductance; $\mu \mathrm{S} / \mathrm{cm}$, microsiemens per centimeter; ${ }^{\circ} \mathrm{C}$, degrees Celsius; diss., dissolved; $\mathrm{mg} / \mathrm{L}$, milligrams per liter; $\mathrm{pH}$ in standard units; -, no measurement made; >, greater than; $\mathrm{U} / \mathrm{S}$, upstream; $\mathrm{D} / \mathrm{S}$, downstream; R, river]

\begin{tabular}{|c|c|c|c|c|c|c|c|c|c|c|c|c|}
\hline \multirow[b]{2}{*}{ Station identifier } & \multirow[b]{2}{*}{ Station name } & \multirow[b]{2}{*}{$\begin{array}{l}\text { Alternate } \\
\text { station } \\
\text { identifier }\end{array}$} & \multirow{2}{*}{$\begin{array}{l}\text { Latitude } \\
\text { (decimal } \\
\text { degrees, } \\
\text { UTM83) }\end{array}$} & \multirow{2}{*}{$\begin{array}{c}\text { Longitude } \\
\text { (decimal } \\
\text { degrees, } \\
\text { UTM83) }\end{array}$} & \multirow{2}{*}{$\begin{array}{c}\text { River miles } \\
\text { downstream } \\
\text { from station } \\
09504000\end{array}$} & \multicolumn{7}{|c|}{ June 20-21, 2007} \\
\hline & & & & & & Date & Time & $\begin{array}{c}\text { Discharge } \\
\left(\mathrm{ft}^{3} / \mathrm{s}\right)\end{array}$ & $\begin{array}{c}\text { Specific } \\
\text { cond. } \\
(\mu \mathrm{S} / \mathrm{cm})\end{array}$ & $\begin{array}{l}\text { Water } \\
\text { temp. } \\
\left({ }^{\circ} \mathrm{C}\right)\end{array}$ & $\begin{array}{c}\text { Diss. } \\
\text { oxygen } \\
\text { (mg/L) }\end{array}$ & $\mathrm{pH}$ \\
\hline 343502111511100 & BEAVER CREEK BELOW EUREKA DITCH RETURN FLOW & $56 B$ & 34.583913 & -111.853763 & - & $6 / 20$ & - & $0 ; 3.8^{e}$ & - & - & - & - \\
\hline 343425111511200 & VERDE R BELOW BEAVER CREEK & 57 & 34.573691 & -111.854040 & 35.3 & $6 / 21$ & $17: 15$ & 35 & 815 & 25.8 & 11.4 & 7.9 \\
\hline 343018111505100 & DIAMOND S DITCH LEFT BANK & 59 & 34.555164 & -111.848345 & - & $6 / 21$ & $18: 42$ & 28 & 785 & 24.9 & 14.3 & 8.1 \\
\hline 343259111505700 & DIAMOND S DITCH RETURN FLOW AT WHITE BRIDGE & 60 & 34.549858 & -111.849901 & - & $6 / 21$ & 17:09 & $0.9 ; 0.8$ & 780 & 25.6 & 9.7 & 8.2 \\
\hline 343259111510500 & VERDE R 0.25 MILES BELOW STATION 09505550 & 61 & 34.549747 & -111.852095 & 37.5 & $6 / 21$ & $10: 45$ & 12 & 773 & 22.0 & 9.3 & 8.1 \\
\hline 343242111512700 & RIVER PUMP LEFT BANK 0.5 MILES BELOW WHITE BRIDGE & $61 B$ & 34.545247 & -111.858262 & - & $6 / 21$ & - & $>0^{f}$ & - & - & - & - \\
\hline 343242111513500 & VERDE R 0.75 MILES BELOW WHITE BRIDGE & $61 \mathrm{C}$ & 34.544970 & -111.860318 & 38.1 & $6 / 21$ & $11: 45$ & 9.0 & - & - & - & - \\
\hline 343156111520600 & VERDE R U/S OF RYAL CANYON TURNOUT & 62 & 34.532164 & -111.869096 & 39.2 & $6 / 21$ & $13: 30$ & 11 & 1060 & 27.1 & 12.2 & 8.3 \\
\hline 343124111500400 & VERDE R ABOVE DIAMOND S FINAL WASTE NEAR CAMP VERDE & 63 & 34.523359 & -111.835150 & 41.3 & $6 / 21$ & $17: 12$ & $15 ; 15$ & 1270 & 26.5 & 12.9 & 8.4 \\
\hline 343123111500100 & DIAMOND S DITCH FINAL WASTEWAY NEAR CAMP VERDE & $63 \mathrm{~A}$ & 34.523081 & -111.834317 & - & $6 / 21$ & 17:10 & 16 & 756 & 26.1 & 7.7 & 8.3 \\
\hline 343021111501000 & VERDE R ABOVE WEST CLEAR CREEK 100 FEET D/S OF PUMP & 65 & 34.505915 & -111.836677 & 42.8 & $6 / 21$ & $10: 40$ & $36 ; 36$ & 1110 & 22.6 & 7.3 & 7.3 \\
\hline 343019111493900 & WEST CLEAR CREEK ABOVE CONFLUENCE NEAR CAMP VERDE & $65 B$ & 34.505304 & -111.828205 & - & - & - & - & - & - & - & - \\
\hline 342953111485500 & VERDE R AT CLIFFS U/S OF VERDE DITCH RETURN & 66 & 34.497971 & -111.816093 & 44.0 & $6 / 21$ & $12: 55$ & 34 & 1170 & 24.9 & 8.0 & 8.1 \\
\hline 342913111490100 & VERDE R 1.6 MILES BELOW WEST CLEAR CREEK & 67 & 34.486971 & -111.817649 & 45.2 & $6 / 21$ & $15: 25$ & 38 & 1200 & 26.1 & 9.2 & 8.3 \\
\hline 342847111484500 & VERDE DITCH RETURN FLOW & $67 A$ & 34.479749 & -111.813204 & - & $6 / 21$ & 16:15 & $>0$ & 530 & 26.9 & 6.6 & 8.3 \\
\hline 342847111484300 & VERDE R 50 FEET D/S OF VERDE DITCH RETURN FLOW & $67 \mathrm{~B}$ & 34.479749 & -111.812649 & 45.5 & $6 / 21$ & $16: 05$ & - & 1150 & 26.3 & 9.1 & 8.3 \\
\hline 342848111475700 & VERDE R AT BEASLEY FLAT NEAR CAMP VERDE & 68 & 34.480026 & -111.799871 & 46.7 & $6 / 21$ & 10:08 & 52 & 1140 & 24.2 & 8.7 & 8.2 \\
\hline 342749111471100 & VERDE R ABOVE THE FALLS NEAR CAMP VERDE & 79 & 34.463638 & -111.787093 & 49.2 & - & - & - & - & - & - & - \\
\hline 09506000 & VERDE R NEAR CAMP VERDE & 70 & 34.448361 & -111.789870 & 50.9 & - & - & $41^{\mathrm{g}}$ & - & - & - & - \\
\hline
\end{tabular}

a Diversion upstream of this station could have been diverting water (diversion amount estimated at $2 \mathrm{ft}^{3} / \mathrm{s}$ in Feb 2011. Not measured in June 2007).

bEstimated by using the float method (Weight and Sonderegger, 2001, p. 225).

'Poor quality measurement. Repeat measurement shown here used as actual measured value

${ }^{\mathrm{d}}$ Very low flow, poorly channelized. This is a visual estimate.

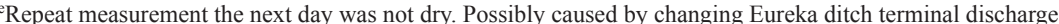

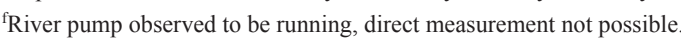

${ }^{g}$ Direct measurement not made; value is mean of daily mean values published for the period of the base-flow evaluation. 
Table 1. Synoptic base-flow measurements for the Verde River, tributary confluences with the Verde River, and major ditch diversions, June 2007 and February 2011 , Verde Valley, central Arizona.-Continued

[Italicized rows indicate measurements not on Verde River mainstem; multiple values indicate repeat measurements for quality assurance; UTM83, Universal Transverse Mercator 1983; ft ${ }^{3} / \mathrm{s}$, cubic feet per second; cond., conductance; $\mu \mathrm{S} / \mathrm{cm}$, microsiemens per centimeter; ${ }^{\circ} \mathrm{C}$, degrees Celsius; diss., dissolved; $\mathrm{mg} / \mathrm{L}$, milligrams per liter; $\mathrm{pH}$ in standard units; -, no measurement made; >, greater than; $\mathrm{U} / \mathrm{S}$, upstream; $\mathrm{D} / \mathrm{S}$, downstream; R, river]

\begin{tabular}{|c|c|c|c|c|c|c|c|c|c|c|c|c|}
\hline \multirow[b]{2}{*}{ Station identifier } & \multirow[b]{2}{*}{ Station name } & \multirow[b]{2}{*}{$\begin{array}{l}\text { Alternate } \\
\text { station } \\
\text { identifier }\end{array}$} & \multirow{2}{*}{$\begin{array}{l}\text { Latitude } \\
\text { (decimal } \\
\text { degrees, } \\
\text { UTM83) }\end{array}$} & \multirow{2}{*}{$\begin{array}{c}\text { Longitude } \\
\text { (decimal } \\
\text { degrees, } \\
\text { UTM83) }\end{array}$} & \multirow{2}{*}{$\begin{array}{c}\text { River miles } \\
\text { downstream } \\
\text { from station } \\
09504000\end{array}$} & \multicolumn{7}{|c|}{ February 1-3, 2011} \\
\hline & & & & & & Date & Time & $\begin{array}{c}\text { Discharge } \\
\left(\mathrm{ft}^{3} / \mathrm{s}\right)\end{array}$ & $\begin{array}{c}\text { Specific } \\
\text { cond. } \\
(\mu \mathrm{S} / \mathrm{cm})\end{array}$ & $\begin{array}{l}\text { Water } \\
\text { temp. } \\
\left({ }^{\circ} \mathrm{C}\right)\end{array}$ & $\begin{array}{c}\text { Diss. } \\
\text { oxygen } \\
\text { (mg/L) }\end{array}$ & $\mathrm{pH}$ \\
\hline 09504000 & VERDE R NEAR CLARKDALE & 1 & 34.852242 & -112.065994 & 0.0 & $2 / 1$ & 17:03 & 72 & 524 & 11.2 & 9.9 & 8.2 \\
\hline 345058112034700 & VERDE R ABOUT 600 FEET BELOW 09504000 & 2 & 34.849464 & -112.063772 & 0.3 & - & - & - & - & - & - & - \\
\hline 345004112025400 & VERDE R 1.5 MILES D/S OF GAGE 09504000 & 3 & 34.834464 & -112.049049 & 1.9 & $2 / 1$ & $14: 28$ & 76 & 521 & 10.8 & 10.5 & 8.4 \\
\hline 344842112032800 & VERDE R ABOVE OLD DUFF DITCH & 4 & 34.811687 & -112.058493 & 5.3 & - & - & - & - & - & - & - \\
\hline 344831112031900 & VERDE R AT REITZ RANCH NEAR CLARKDALE & $4 \mathrm{~A}$ & 34.808722 & -112.055361 & 4.7 & $2 / 1$ & 10:55 & 76 & 515 & 9.4 & 10.2 & 8.5 \\
\hline 344807112024400 & VERDE R BELOW PHELPS DODGE PUMPING STATION & 5 & 34.801965 & -112.046270 & 5.5 & - & - & $-{ }^{\mathrm{a}}$ & - & - & - & - \\
\hline 344742112033100 & VERDE R 0.5 MILES ABOVE SLAG PILE & 6 & 34.795020 & -112.059326 & 7.4 & $2 / 3$ & 10:07 & 77 & 524 & 3.6 & - & 8.5 \\
\hline 344652112024500 & PECKS LAKE DIVERSION FROM VERDE RIVER NEAR CLARKDALE & $7 B$ & 34.781132 & -112.046548 & - & - & - & $0^{h}$ & - & - & - & - \\
\hline 344640112025600 & VERDE R BELOW TAVASCI DITCH & 8 & 34.777798 & -112.049603 & 9.1 & $2 / 3$ & $12: 12$ & 82 & 521 & 4.8 & - & 8.5 \\
\hline 344635112024900 & VERDE R ABOUT 400 FEET D/S OF BITTER CREEK & 9 & 34.776409 & -112.047659 & 9.2 & - & - & - & - & - & - & - \\
\hline 344605112022400 & VERDE R 800 FEET U/S OF TUZIGOOT BRIDGE & 10 & 34.768076 & -112.040714 & 9.9 & $2 / 3$ & 13:17 & $77 ; 78^{\mathrm{i}}$ & 536 & 5.5 & - & 8.5 \\
\hline 344550112020400 & HICKEY DITCH 100 FEET D/S FROM GATE & 11 & 34.763910 & -112.035158 & - & $2 / 3$ & $9: 53$ & 15 & 554 & 4.7 & - & 8.5 \\
\hline 344555112020900 & VERDE R BELOW HICKEY DITCH & $12 \mathrm{~A}$ & 34.765215 & -112.036519 & 10.2 & $2 / 3$ & 11:08 & $79 ; 76 ; 66^{\mathrm{i}}$ & 549 & 5.2 & - & 8.5 \\
\hline 344554112013500 & COTTONWOOD DITCH BELOW HICKEY DITCH FLUME & 14 & 34.765021 & -112.027102 & - & - & - & $>0$ & - & - & - & - \\
\hline 344558112013600 & VERDE R BELOW COTTONWOOD DITCH & 15 & 34.766215 & -112.027241 & 10.8 & $2 / 2$ & 10:10 & 44 & 543 & 4.3 & 11.4 & 8.1 \\
\hline 344559112011800 & VERDE R ABOVE TAVASCI WASH & 16 & 34.766020 & -112.022435 & 11.1 & - & - & - & - & - & - & - \\
\hline 344600112011700 & TAVASCI MARSH OUTFLOW & $16 \mathrm{~A}$ & 34.766694 & -112.021306 & - & $2 / 2$ & $12: 20$ & 0.9 & 723 & 2.5 & 9.5 & 7.3 \\
\hline 344556112011700 & VERDE R BELOW TAVASCI WASH & 17 & 34.765604 & -112.022463 & 11.2 & - & - & - & - & - & - & - \\
\hline 344545112012300 & HICKEY DITCH ABOVE VERDE R FLUME NEAR CLARKDALE & - & 34.762521 & -112.023769 & - & $2 / 3$ & 12:00 & $6^{b}$ & - & - & - & - \\
\hline 344542112011800 & HICKEY DITCH BELOW VERDE R FLUME NEAR CLARKDALE & - & 34.761687 & -112.022380 & - & $2 / 3$ & $12: 30$ & $4^{b}$ & - & - & - & - \\
\hline 344528112014200 & VERDE R ABOVE MESCAL GULCH & 18 & 34.757771 & -112.029130 & 11.9 & - & - & - & - & - & - & - \\
\hline 344516112013900 & VERDE R BELOW MESCAL GULCH & 19 & 34.754465 & -112.028158 & 12.2 & - & - & - & - & - & - & - \\
\hline 344504112010000 & VERDE R BELOW DEADHORSE BRIDGE & 20 & 34.750854 & -112.020713 & 12.8 & $2 / 2$ & $14: 40$ & 47 & 539 & 6.1 & 11.2 & 8.2 \\
\hline 344504112005600 & VERDE R ABOVE QUAIL CREEK & 21 & 34.751132 & -112.016268 & 13.0 & $2 / 1$ & $12: 27$ & 49 & 530 & 8.9 & 9.5 & 8.9 \\
\hline 344505112005200 & HICKEY DITCH RETURN FLOW NEAR QUAIL CREEK & 22 & 34.751521 & -112.015102 & - & $2 / 1$ & $11: 32$ & 0.2 & 508 & 7.3 & 11.2 & 7.9 \\
\hline 344458112003300 & VERDE R ABOVE COTTONWOOD DITCH RETURN FLOWS $1 \& 2$ & 23 & 34.749465 & -112.009824 & 13.4 & $2 / 1$ & $14: 39$ & 49 & 540 & 10.1 & 10.0 & 7.7 \\
\hline 344458112003100 & VERDE R BELOW COTTONWOOD DITCH RETURN FLOWS $1 \& 2$ & 24 & 34.749382 & -112.009296 & 13.4 & $2 / 1$ & 16:00 & 53 & 545 & 10.2 & 10.0 & 8.0 \\
\hline 344504112000300 & VERDE R ABOVE HICKEY DITCH RETURN FLOW & $25 \mathrm{~A}$ & 34.751048 & -112.001712 & 13.9 & $2 / 1$ & $17: 30$ & - & 543 & 10.1 & 10.0 & 8.0 \\
\hline 344504112000400 & HICKEY DITCH RETURN FLOW & 26 & 34.751076 & -112.001768 & - & $2 / 1$ & 18:01 & 0.7 & 505 & 8.4 & 10.6 & - \\
\hline 344443111594500 & VERDE R ABOUT 0.5 MILES U/S OF MINGUS BRIDGE & 27 & 34.745243 & -111.996434 & 14.5 & - & - & - & - & - & - & - \\
\hline 344420111595500 & VERDE R BELOW MINGUS BRIDGE & 28 & 34.738910 & -111.999323 & 15.0 & $2 / 1$ & 11:53 & 56 & 574 & 8.9 & 9.9 & 8.2 \\
\hline 344404112000000 & VERDE R ABOVE MOUTH OF SPRING WASH GULCH & 29 & 34.734938 & -112.000795 & 15.3 & $2 / 1$ & 14:42 & 57 & 574 & 10.2 & 10.5 & 8.3 \\
\hline 344357111595600 & COTTONWOOD DITCH RETURN FLOW AT GREENWAYLAND & 30 & 34.732438 & -111.999573 & - & $2 / 1$ & 14:40 & $0.4^{b}$ & 522 & 8.4 & 12.5 & 8.8 \\
\hline 344331111593100 & COTTONWOOD DITCH RETURN FLOW 0.3 MILES U/S OF HIGHWAY $89 \mathrm{~A}$ & $30 A$ & 34.725243 & -111.992740 & - & - & - & - & - & - & - & - \\
\hline 344329111593100 & COTTONWOOD DITCH RETURN FLOW 80 FEET D/S OF SITE $30 \mathrm{~A}$ & $30 B$ & 34.724854 & -111.992767 & - & $2 / 1$ & 16:00 & $1.5^{b}$ & - & - & - & - \\
\hline
\end{tabular}


Table 1. Synoptic base-flow measurements for the Verde River, tributary confluences with the Verde River, and major ditch diversions, June 2007 and February 2011, Verde Valley, central Arizona.-Continued

[Italicized rows indicate measurements not on Verde River mainstem; multiple values indicate repeat measurements for quality assurance; UTM83, Universal Transverse Mercator 1983 ; ft $3 \mathrm{~s}^{\mathrm{s}}$, cubic feet per second; cond., conductance; $\mathrm{\mu S} / \mathrm{cm}$, microsiemens per centimeter; ${ }^{\circ} \mathrm{C}$, degrees Celsius; diss., dissolved; $\mathrm{mg} / \mathrm{L}$, milligrams per liter; $\mathrm{pH}$ in standard units; -, no measurement made; >, greater than; $\mathrm{U} / \mathrm{S}$, upstream; $\mathrm{D} / \mathrm{S}$, downstream; R, river]

\begin{tabular}{|c|c|c|c|c|c|c|c|c|c|c|c|c|}
\hline \multirow[b]{2}{*}{ Station identifier } & \multirow[b]{2}{*}{ Station name } & \multirow[b]{2}{*}{$\begin{array}{c}\text { Alternate } \\
\text { station } \\
\text { identifier }\end{array}$} & \multirow[b]{2}{*}{$\begin{array}{l}\text { Latitude } \\
\text { (decimal degrees, } \\
\text { UTM83) }\end{array}$} & \multirow[b]{2}{*}{$\begin{array}{l}\text { Longitude (decimal } \\
\text { degrees, UTM83) }\end{array}$} & \multirow{2}{*}{$\begin{array}{c}\text { River miles } \\
\text { downstream } \\
\text { from station } \\
09504000\end{array}$} & \multicolumn{7}{|c|}{ February 1-3, 2011} \\
\hline & & & & & & Date & Time & $\begin{array}{c}\text { Discharge } \\
\left(\mathrm{ft}^{3} / \mathrm{s}\right)\end{array}$ & $\begin{array}{c}\text { Specific } \\
\text { cond. } \\
(\mu \mathrm{S} / \mathrm{cm})\end{array}$ & $\begin{array}{l}\text { Water } \\
\text { temp. } \\
\left({ }^{\circ} \mathrm{C}\right)\end{array}$ & $\begin{array}{c}\text { Diss. } \\
\text { oxygen } \\
\text { (mg/L) }\end{array}$ & $\mathrm{pH}$ \\
\hline 344327111592900 & VERDE R ABOUT 0.25 MILES U/S OF HIGHWAY 89A BRIDGE & 31 & 34.724104 & -111.992017 & 16.3 & $2 / 1$ & 17:06 & 60 & 533 & - & - & - \\
\hline 344319111592200 & COTTONWOOD DITCH RETURN FLOW 70 FEET D/S OF HIGHWAY $89 A$ & $31 B$ & 34.721069 & -111.990489 & - & $2 / 3$ & 13:00 & 0 & - & - & - & - \\
\hline 344318111592400 & VERDE R AT HIGHWAY 89A NEAR COTTONWOOD & 32 & 34.720299 & -111.990545 & 16.6 & - & - & - & - & - & - & - \\
\hline 344232111590400 & VERDE R 500 FEET ABOVE END OF COTTONWOOD DITCH & 33 & 34.708910 & -111.985156 & 17.4 & $2 / 3$ & $14: 10$ & $80 ; 83$ & 532 & 4.6 & 12.1 & 8.4 \\
\hline 344229111585800 & END OF COTTONWOOD DITCH SITE 34 & 34 & 34.708077 & -111.983489 & - & $2 / 3$ & 14:00 & 0 & - & - & - & - \\
\hline 344228111584300 & VERDE R BELOW END OF COTTONWOOD DITCH & 35 & 34.708077 & -111.979323 & 17.8 & $2 / 3$ & $12: 31$ & 88 & 540 & 4.1 & 12.0 & 8.3 \\
\hline 344158111574000 & VERDE R AT HEAD OF 2ND BEND BELOW COTTONWOOD DITCH & 36 & 34.699466 & -111.961822 & 19.2 & $2 / 3$ & $9: 37$ & 85 & 543 & 3.4 & 11.9 & 8.2 \\
\hline 344125111575400 & VERDE R AT TAIL OF 2ND BEND ABOVE SPRING & 37 & 34.690383 & -111.965822 & 20.7 & - & - & - & - & - & - & - \\
\hline 344106111574700 & VERDE R AT BLACK MESA TANK 1.3 MILES U/S OF OAK CREEK & 38 & 34.685022 & -111.963767 & 21.1 & $2 / 1$ & $10: 52$ & 89 & - & 8.0 & 9.5 & 9.0 \\
\hline 344026111565000 & VERDE R ABOUT 0.7 MILES U/S OF MOUTH OF OAK CREEK & $38 \mathrm{~B}$ & 34.673967 & -111.947877 & 22.5 & $2 / 1$ & $12: 31$ & 86 & 593 & 5.2 & 12.6 & 8.3 \\
\hline 344044111563200 & VERDE R ABOVE SPRINGS ABOVE MOUTH OF OAK CREEK & 39 & 34.678855 & -111.942877 & 23.1 & - & - & - & - & - & - & - \\
\hline 344045111562400 & OAK CREEK AT VERDE R NEAR CORNVILLE,AZ & $40 B$ & 34.679189 & -111.940710 & - & $2 / 1$ & 15:02 & $72 ; 76^{j}$ & 432 & 5.8 & 12.3 & 8.3 \\
\hline 344032111562300 & VERDE R ABOUT 1000 FEET D/S OF MOUTH OF OAK CREEK & 41 & 34.675689 & -111.940572 & 23.3 & $2 / 1$ & $14: 03$ & 166 & 583 & 5.4 & 12.5 & 8.4 \\
\hline 343956111561100 & OK DITCH 600 FEET D/S FROM THE HEAD & 43 & 34.665578 & -111.937099 & - & - & - & - & - & - & - & - \\
\hline 343843111555500 & VERDE R BELOW OK DITCH TURNOUT NEAR CORNVILLE & 42 & 34.645301 & -111.932654 & 25.6 & $2 / 1$ & 10:58 & 158 & 489 & 8.3 & 10.6 & 8.4 \\
\hline 343843111554700 & OK DITCH 1.65 MILES BELOW HEADGATE NEAR CAMP VERDE & $43 D$ & 34.645301 & -111.930432 & - & $2 / 1$ & $12: 12$ & 5.3 & 486 & 8.8 & 10.9 & 8.3 \\
\hline 343826111554700 & VERDE R U/S OF HAYFIELD DRAW & 44 & 34.640551 & -111.930404 & 24.1 & - & - & - & - & - & - & - \\
\hline 343818111553000 & VERDE R D/S OF HAYFIELD DRAW & $44 \mathrm{~A}$ & 34.638440 & -111.925654 & 26.3 & $2 / 1$ & 14:18 & 149 & 486 & 9.0 & 10.8 & 8.4 \\
\hline 343751111545700 & VERDE R BELOW CHERRY CREEK AND ABOVE EUREKA DITCH & 45 & 34.630884 & -111.916543 & 27.1 & - & - & - & - & - & - & - \\
\hline 343758111534600 & EUREKA DITCH AT HEAD NEAR CAMP VERDE & $45 A A$ & 34.632801 & -111.896820 & - & - & - & $0^{k}$ & - & - & - & - \\
\hline 343722111535900 & VERDE R D/S OF FORD & $46 \mathrm{~A}$ & 34.622940 & -111.900459 & 29.0 & $2 / 2$ & 11:14 & 164 & 482 & 6.0 & 11.7 & - \\
\hline 343704111535800 & VERDE R BELOW EUREKA DITCH D/S OF FORD & 47 & 34.617662 & -111.900098 & 29.4 & - & - & - & - & - & - & - \\
\hline 343629111523500 & VERDE R BELOW VERDE DITCH & 49 & 34.608163 & -111.877097 & 31.5 & - & - & - & - & - & - & - \\
\hline 343626111523700 & VERDE R D/S OF VERDE DITCH & $47 \mathrm{~N}$ & 34.607246 & -111.877541 & 31.7 & $2 / 1$ & $16: 45$ & 154 & 491 & 9.0 & 10.8 & 8.4 \\
\hline 343558111524000 & VERDE R ABOVE GRIEF HILL WASH & 50 & 34.599468 & -111.878569 & 32.1 & - & - & - & - & - & - & - \\
\hline 343529111530400 & VERDE DITCH RETURN FLOW & $50 \mathrm{~A}$ & 34.591413 & -111.885152 & - & - & - & $0^{k}$ & - & - & - & - \\
\hline 343519111525500 & VERDE R BELOW GRIEF HILL WASH & 51 & 34.588607 & -111.882763 & 32.9 & - & - & - & - & - & - & - \\
\hline 343513111524600 & VERDE R AT I-17 BRIDGE NEAR CAMP VERDE & 52 & 34.586969 & -111.880152 & 33.4 & $2 / 2$ & $13: 42$ & 157 & 490 & 6.4 & 11.8 & 6.7 \\
\hline 343450111523600 & VERDE DITCH RETURN FLOW AT GADDIS WASH NEAR CAMP VERDE & 53 & 34.580580 & -111.877374 & - & - & - & $0^{k}$ & - & - & - & - \\
\hline 343431111515700 & VERDE R ABOVE EUREKA DITCH RETURN FLOW & 54 & 34.575219 & -111.866624 & 34.5 & $2 / 2$ & $17: 43$ & 158 & 498 & 6.3 & 11.8 & 8.5 \\
\hline 343434111515400 & EUREKA DITCH RETURN FLOW BELOW I-17 NEAR CAMP VERDE & $54 A$ & 34.576135 & -111.865707 & - & $2 / 2$ & $17: 30$ & 0 & - & - & - & - \\
\hline 343427111513800 & VERDE R ABOVE SPRING AT BLACK BRIDGE & 55 & 34.574191 & -111.861318 & 34.8 & - & - & - & - & - & - & - \\
\hline 343424111513300 & VERDE R ABOVE BEAVER CREEK NEAR CAMP VERDE & $55 \mathrm{~A}$ & 34.573358 & -111.859874 & 34.9 & - & - & - & - & - & - & - \\
\hline 343424111512200 & VERDE R 600 FEET ABOVE BEAVER CREEK AT CAMP VERDE & 56 & 34.573358 & -111.856818 & 35.1 & $2 / 2$ & $12: 32$ & 165 & 553 & 6.9 & 11.1 & 7.9 \\
\hline
\end{tabular}


Table 1. Synoptic base-flow measurements for the Verde River, tributary confluences with the Verde River, and major ditch diversions, June 2007 and February 2011, Verde Valley, central Arizona.-Continued

[Italicized rows indicate measurements not on Verde River mainstem; multiple values indicate repeat measurements for quality assurance; UTM83, Universal Transverse Mercator 1983; ft ${ }^{3} / \mathrm{s}$, cubic feet per second; cond., conductance; $\mu \mathrm{S} / \mathrm{cm}$, microsiemens per centimeter; ${ }^{\circ} \mathrm{C}$, degrees Celsius; diss., dissolved; $\mathrm{mg} / \mathrm{L}$, milligrams per liter; $\mathrm{pH}$ in standard units; -, no measurement made; >, greater than; $\mathrm{U} / \mathrm{S}$, upstream; $\mathrm{D} / \mathrm{S}$, downstream; R, river]

\begin{tabular}{|c|c|c|c|c|c|c|c|c|c|c|c|c|}
\hline \multirow[b]{2}{*}{ Station identifier } & \multirow[b]{2}{*}{ Station name } & \multirow[b]{2}{*}{$\begin{array}{c}\text { Alternate } \\
\text { station } \\
\text { identifier }\end{array}$} & \multirow[b]{2}{*}{$\begin{array}{l}\text { Latitude } \\
\text { (decimal degrees, } \\
\text { UTM83) }\end{array}$} & \multirow[b]{2}{*}{$\begin{array}{l}\text { Longitude (decimal } \\
\text { degrees, UTM83) }\end{array}$} & \multirow{2}{*}{$\begin{array}{c}\text { River miles } \\
\text { downstream } \\
\text { from station } \\
09504000\end{array}$} & \multirow[b]{2}{*}{ Date } & \multicolumn{6}{|c|}{ February 1-3, 2011} \\
\hline & & & & & & & Time & $\begin{array}{c}\text { Discharge } \\
\left(\mathrm{ft}^{3} / \mathrm{s}\right)\end{array}$ & $\begin{array}{c}\text { Specific } \\
\text { cond. } \\
(\mu \mathrm{S} / \mathrm{cm})\end{array}$ & $\begin{array}{l}\text { Water } \\
\text { temp. } \\
\left({ }^{\circ} \mathrm{C}\right)\end{array}$ & $\begin{array}{c}\text { Diss. } \\
\text { oxygen } \\
\text { (mg/L) }\end{array}$ & $\mathrm{pH}$ \\
\hline 343506111511300 & BEAVER CREEK ABOVE EUREKA DITCH NEAR CAMP VERDE & $56 A$ & 34.586746 & -111.855235 & - & $2 / 3$ & 13:48 & 2 & 475 & 6.0 & 10.3 & 8.0 \\
\hline 343502111511100 & BEAVER CREEK BELOW EUREKA DITCH RETURN FLOW & $56 B$ & 34.583913 & -111.853763 & - & - & - & - & - & - & - & - \\
\hline 343425111511200 & VERDE R BELOW BEAVER CREEK & 57 & 34.573691 & -111.854040 & 35.3 & $2 / 2$ & 15:19 & 182 & 519 & 7.3 & 11.7 & 8.3 \\
\hline 343018111505100 & DIAMOND S DITCH LEFT BANK & 59 & 34.555164 & -111.848345 & - & - & - & $0^{k}$ & - & - & - & - \\
\hline 343259111505700 & DIAMOND S DITCH RETURN FLOW AT WHITE BRIDGE & 60 & 34.549858 & -111.849901 & - & - & - & $0^{k}$ & - & - & - & - \\
\hline 343259111510500 & VERDE R 0.25 MILES BELOW STATION 09505550 & 61 & 34.549747 & -111.852095 & 37.5 & $2 / 2$ & $10: 52$ & $174 ; 178$ & 515 & 6.3 & 9.0 & 8.1 \\
\hline 343242111512700 & RIVER PUMP LEFT BANK 0.5 MILES BELOW WHITE BRIDGE & $61 B$ & 34.545247 & -111.858262 & - & - & - & $0^{k}$ & - & - & - & - \\
\hline 343242111513500 & VERDE R 0.75 MILES BELOW WHITE BRIDGE & $61 \mathrm{C}$ & 34.544970 & -111.860318 & 38.1 & - & - & - & - & - & - & - \\
\hline 343156111520600 & VERDE R U/S OF RYAL CANYON TURNOUT & 62 & 34.532164 & -111.869096 & 39.2 & - & - & - & - & - & - & - \\
\hline 343124111500400 & VERDE R ABOVE DIAMOND S FINAL WASTE NEAR CAMP VERDE & 63 & 34.523359 & -111.835150 & 41.3 & $2 / 3$ & $11: 25$ & 189 & 609 & 5.2 & 11.6 & 8.2 \\
\hline 343123111500100 & DIAMOND S DITCH FINAL WASTEWAY NEAR CAMP VERDE & $63 A$ & 34.523081 & -111.834317 & - & $2 / 3$ & 11:00 & 0 & - & - & - & - \\
\hline 343021111501000 & VERDE R ABOVE WEST CLEAR CREEK 100 FEET D/S OF PUMP & 65 & 34.505915 & -111.836677 & 42.8 & - & - & - & - & - & - & - \\
\hline 343019111493900 & WEST CLEAR CREEK ABOVE CONFLUENCE NEAR CAMP VERDE & $65 B$ & 34.505304 & -111.828205 & - & $2 / 2$ & 10:37 & 10 & 418 & 2.9 & - & 8.3 \\
\hline 342953111485500 & VERDE R AT CLIFFS U/S OF VERDE DITCH RETURN & 66 & 34.497971 & -111.816093 & 44.0 & $2 / 2$ & 13:57 & 214 & 560 & 7.1 & - & 8.3 \\
\hline 342913111490100 & VERDE R 1.6 MILES BELOW WEST CLEAR CREEK & 67 & 34.486971 & -111.817649 & 45.2 & - & - & - & - & - & - & - \\
\hline 342847111484500 & VERDE DITCH RETURN FLOW & $67 A$ & 34.479749 & -111.813204 & - & - & - & $0^{k}$ & - & - & - & - \\
\hline 342847111484300 & VERDE R 50 FEET D/S OF VERDE DITCH RETURN FLOW & $67 \mathrm{~B}$ & 34.479749 & -111.812649 & 45.5 & $2 / 2$ & $15: 21$ & 200 & 574 & 7.5 & 9.9 & 8.2 \\
\hline 342848111475700 & VERDE R AT BEASLEY FLAT NEAR CAMP VERDE & 68 & & & 46.7 & $2 / 2$ & 14:00 & 212 & 571 & 6.9 & 10.2 & 8.1 \\
\hline 342749111471100 & VERDE R ABOVE THE FALLS NEAR CAMP VERDE & 79 & 34.463638 & -111.787093 & 49.2 & $2 / 3$ & 11:56 & $201 ; 201$ & 584 & 4.4 & 11.8 & 7.8 \\
\hline 09506000 & VERDE R NEAR CAMP VERDE & 70 & 34.448361 & -111.789870 & 50.9 & - & - & $212^{\mathrm{g}}$ & - & - & - & - \\
\hline
\end{tabular}

\section{hZero flow reported by private landowner.}

iRepeat measurement taken 5 days later (2/8/2011) using same measurement method.

Repeat measurement taken 2 days later (2/3/2011) using same measurement method.

kZero flow observed several times throughout the course of this synoptic base-flow evalution. 


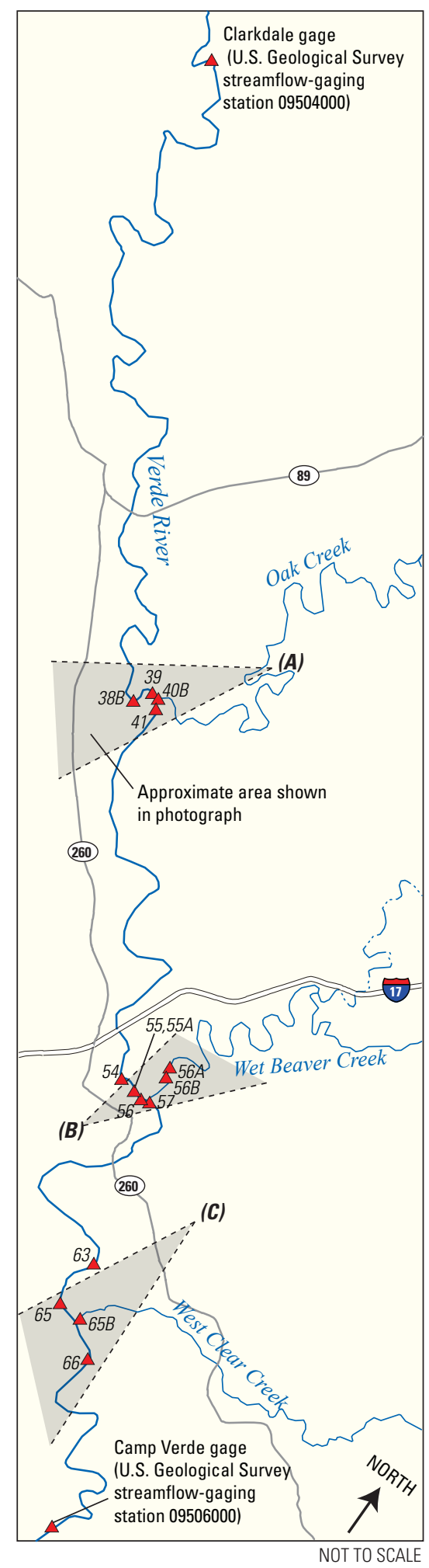

Station numbers correspond to alternate station identifiers in table 1.
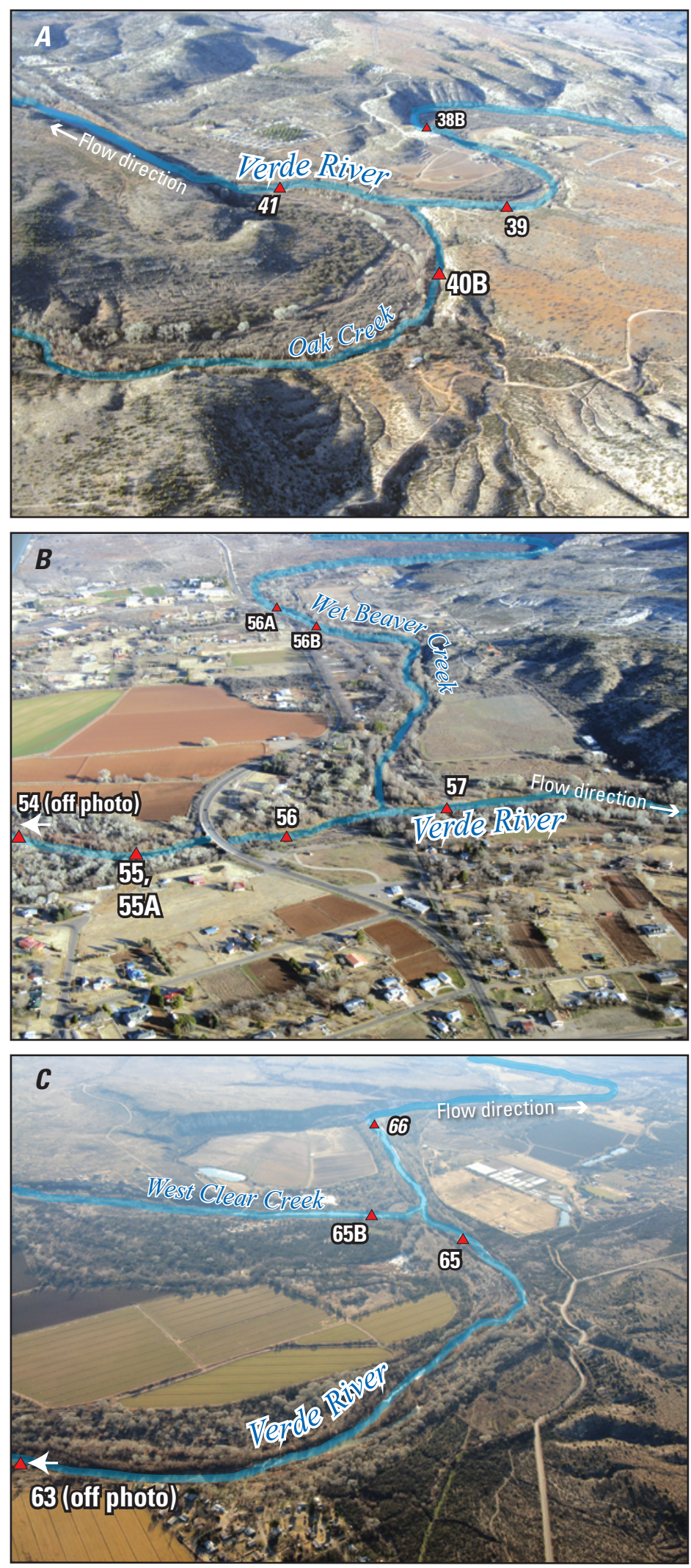

Figure 9. Oblique aerial photographs of the Verde River at the confluences of $(A)$ Oak Creek, $(B)$ Beaver Creek, and (C) West Clear Creek, February 2012, Verde Valley, central Arizona. 
Table 2. Surface-water and groundwater inflows to the Verde River near confluences with perennial tributaries, June 2007 and February 2011, Verde Valley, central Arizona.

$\left[\mathrm{ft}^{3} / \mathrm{s}\right.$, cubic feet per second; $Q_{t r i b}, G W_{\text {netND }}, D_{\text {ret }}$ are variables defined for equations in the main body of the text associated with this table]

\begin{tabular}{lcc}
\hline Verde River inflow component & $\begin{array}{c}\text { Inflow to } \\
\text { Verde River, } \\
\text { June 2007 } \\
\left(\mathbf{f t}^{3} / \mathbf{s}\right)\end{array}$ & $\begin{array}{c}\text { Inflow to } \\
\text { Verde River, } \\
\text { February 2011 } \\
\left(\mathbf{f t}^{3} / \mathbf{s}\right)\end{array}$ \\
\hline \multicolumn{2}{c}{ Confluence of 0 ak Creek } \\
Surface-water inflow $\left(Q_{\text {trib }}\right)$ & 27 to 30 & 72 to 76 \\
Groundwater inflow $\left(G W_{\text {net ND }}\right)$ & 0 & 0 \\
TOTAL & 27 to 30 & 72 to 76 \\
\hline
\end{tabular}

Confluence of Beaver Creek

\begin{tabular}{lcc} 
Surface-water inflow $\left(Q_{\text {trib }}\right)$ & 0 & $2^{\text {a }}$ \\
Groundwater inflow $\left(G W_{\text {netND }}\right)$ & 18 & 22 \\
TOTAL & 18 & 24 \\
\hline
\end{tabular}

\begin{tabular}{lcc}
\hline \multicolumn{3}{c}{ Confluence of West Clear Creek } \\
Surface-water inflow $\left(Q_{\text {trib }}\right)$ & $0^{\text {b }}$ to 3 & 10 \\
Groundwater inflow $\left(G W_{\text {net } N D}\right)$ & $0^{\mathrm{b}}$ to 3 & $0^{\mathrm{b}}$ to 15 \\
Ditch return flow $\left(D_{\text {ret }}\right)$ & $16^{\mathrm{b}}$ to 19 & $0^{\mathrm{b}}$ to 15 \\
TOTAL & $19^{\mathrm{c}}$ & $25^{\mathrm{c}}$ \\
\hline
\end{tabular}

${ }^{a}$ Only one significant figure here, because this measurement was estimated visually.

bPartitioning among flow components for West Clear Creek is uncertain, as there were unmeasured inflow components. All values rounded to whole numbers because of this.

'Despite uncertainly of how inflow components are partitioned, these total values for West Clear Creek are known, as they were calclated directly from flow measurements.

\section{Spatial Variability of Groundwater Fluxes in River Reaches}

A stream can be subdivided into an almost limitless number of smaller reaches to investigate spatial variability of base flow. For this study, the Verde River between the Clarkdale and Camp Verde gages was divided into five stream reaches (see fig. 6). Given the conceptual model employed for this study (see fig. 5), a key restriction for reach delineation was that a reach could not have any component of a ditch diversion entering or leaving it.

The following discussion often refers to net groundwater flux into or out of a reach $\left(G W_{n e t N D}\right)$. As a visual guide for this discussion, refer to figure 10; each data value in the figure represents the measured discharge (shown in fig. 8) minus the sum of upstream tributary surface-water inflows $\left(\Sigma Q_{t r i b}\right)$ to the Verde River for that data value. This data value is referred to as an "adjusted discharge measurement." As discussed in the "Key Sources of Base Flow" section, tributary inflow from Oak, Beaver, and West Clear Creeks is a major contributor to Verde River base flow. When these base-flow contributions are removed as shown in figure 10, other factors that affect base flow become apparent.

\section{Reach I-II}

Reach I-II comprises 7.4 mi of the Verde River (fig.6) that pass through a rugged, lightly populated section of the Verde Valley with relatively little human alteration to the surface-water system. It begins at the upstream end of the study area (the Clarkdale gage) and extends to the last measuring station located upstream of a much more humanaltered portion of the study area (figs. 6 and 8). There are two small ditch diversions in reach I-II, but they were not measured or quantified in either synoptic base-flow survey.

There is evidence of net groundwater discharge to the river in part of this reach: both surveys showed increased flow between river mi 0 and $1.9\left(7.5 \mathrm{ft}^{3} / \mathrm{s}\right.$ in June 2007, $3.7 \mathrm{ft}^{3} / \mathrm{s}$ in February 2011). This observation supports the possibility that this increased streamflow is related to groundwater discharge, but potential measurement uncertainty (10 percent, or about 6 to $8 \mathrm{ft}^{3} / \mathrm{s}$ ) is larger than the measured flow increase. When considered as an entire reach, reach I-II showed a net flow decrease of $1.0 \mathrm{ft}^{3} / \mathrm{s}$ in June 2007 and a net flow increase of $5.2 \mathrm{ft}^{3} / \mathrm{s}$ in February 2011 (table 3 ). This is consistent with the possibility of riparian vegetation intercepting groundwater moving toward the river during the summer before it can discharge to the river. These results also indicate that groundwater gradients near the river could reverse in summer months as compared to winter months. Again, however, the measured changes in flow are within measurement uncertainty, so these indications are not definitive. Additional synoptic base-flow surveys of reach I-II would be helpful in understanding the active processes.

A measurable difference was evident between base flow at the upstream end of this reach (the Clarkdale gage) in June $2007\left(64 \mathrm{ft}^{3} / \mathrm{s}\right)$ and February $2011\left(72 \mathrm{ft}^{3} / \mathrm{s}\right)$. This is consistent with previously measured seasonal variability in base flow here (Blasch and others, 2006, p. 27), and because there are no major surface-water diversions upstream of the Clarkdale gage, seasonal variability likely is caused by seasonal variations in riparian evapotranspiration. Base flow at the Clarkdale gage also varies over longer time periods because of natural recharge rates that vary over decades or longer (Pool and others, 2011); long-term variations in natural recharge produce corresponding fluctuations in water-table altitudes, and therefore affect the rate of base-flow discharge to streams. Superimposed on natural seasonal and decadal base-flow variability also would be any changes in base flow caused by human groundwater withdrawals and incidental recharge upgradient of this reach. Seasonal fluctuations in groundwater pumping also can produce cyclic variations in base flow in connected surface-water streams (Barlow and Leake, in press), but this has not been studied in the Verde River. Additional synoptic base-flow surveys and analyses of the long-term continuous discharge record at the Clarkdale gage could be used to investigate the various periodicities in the base-flow record at the Clarkdale gage. 
Verde River synoptic base-flow surveys, 2007 and 2011, without measured surface-water inflows

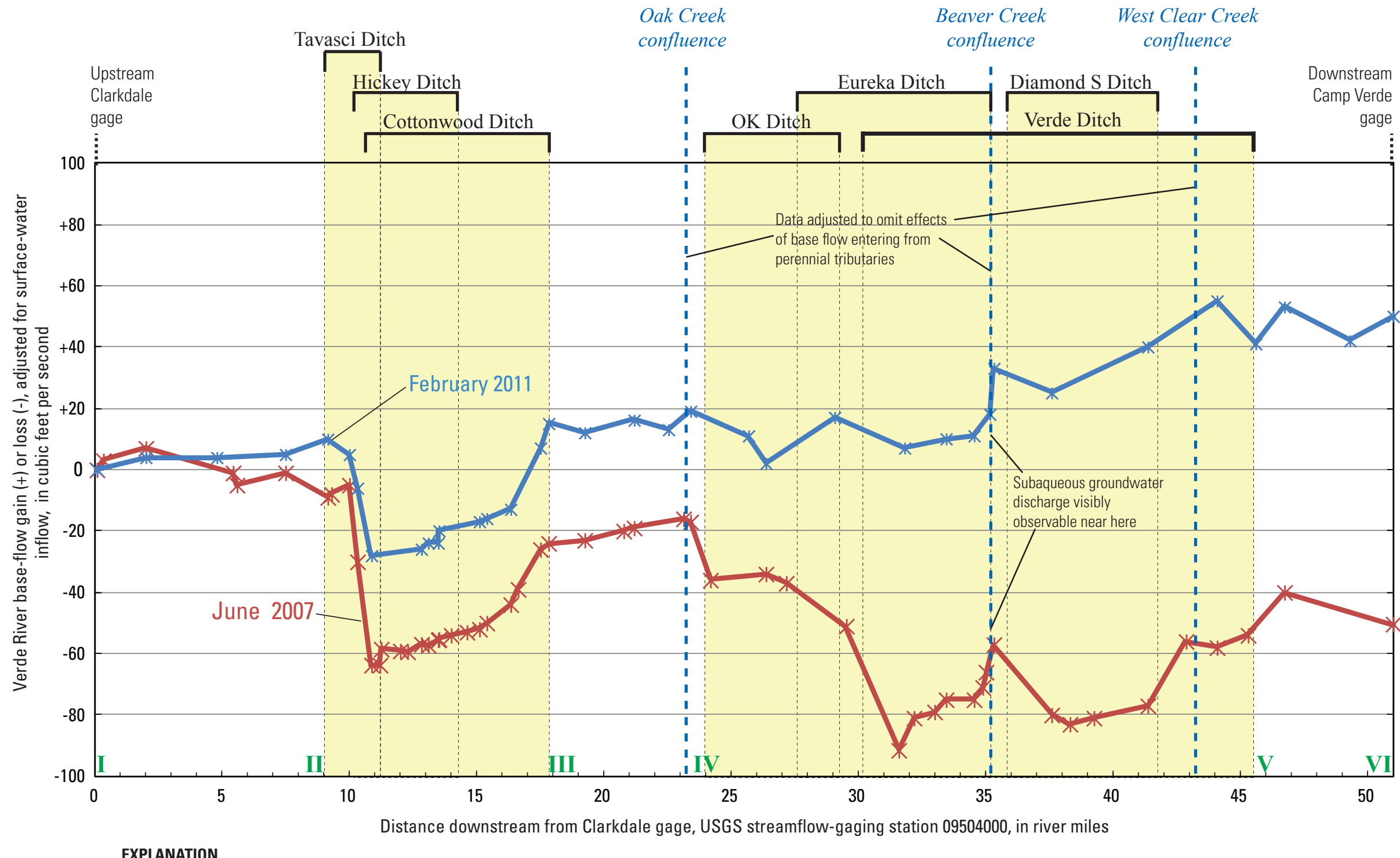

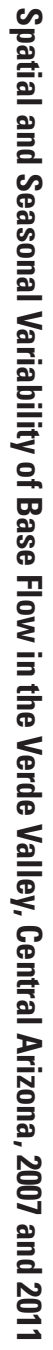

\section{EXPLANATION}

** Adjusted discharge measurements

Hickey Ditch Irrigation ditch name and extent

II Approximate location of start and end of river reach (see also fig. 6)

Figure 10. Synoptic base-flow measurements of the Verde River, adjusted for surface-water inflows, June 2007 and February 2011, Verde Valley, central Arizona. 
Table 3. Measured and calculated flow components for stream reaches along the Verde River, Verde Valley, central Arizona.

[All units cubic feet per second; $Q_{i n}, Q_{\text {out }}, \Sigma Q_{\text {trib }}, \Sigma D_{\text {div }}, \Sigma D_{\text {retMeas }}, G W_{\text {netNDlower }}$, and $G W_{\text {netNDupper }}$ are variables defined for equations in the main body of the text associated with this table]

\begin{tabular}{|c|c|c|c|c|c|c|c|}
\hline $\begin{array}{l}\text { River } \\
\text { reach }^{\mathrm{a}}\end{array}$ & Time period & $\begin{array}{l}\text { Streamflow in } \\
\qquad\left(Q_{i n}\right)\end{array}$ & $\begin{array}{c}\text { Streamflow out } \\
\qquad\left(\boldsymbol{Q}_{\text {out }}\right)\end{array}$ & $\begin{array}{l}\text { Tributary } \\
\text { inflow } \\
\left(\Sigma \boldsymbol{Q}_{\text {trib }}\right)\end{array}$ & $\begin{array}{l}\text { Flow diverted } \\
\text { into ditches } \\
\left(\Sigma D_{\text {div }}\right)\end{array}$ & $\begin{array}{c}\text { Measured } \\
\text { return flows } \\
\text { from ditches } \\
\left(\Sigma D_{\text {retMeas }}\right)\end{array}$ & $\begin{array}{l}\text { Range of net groundwater flux } \\
\left(G W_{\text {netNDlower }} \text { to } G W_{\text {netNDupper }}\right)\end{array}$ \\
\hline I-II & June 2007 & 64 & 63 & 0 & 0 & 0 & $-1.0^{\mathrm{c}}$ \\
\hline I-II & Feb. 2011 & 72 & 77 & 0 & 0 & 0 & $+5.2^{\mathrm{c}}$ \\
\hline II-III & June 2007 & 63 & 40 & 0 & 65 & 14 & -23 to +28 \\
\hline II-III & Feb. 2011 & 77 & 88 & 0 & 37 & 5.7 & +11 to +42 \\
\hline III-IV & June 2007 & 40 & 65 & 30 & 0 & 0 & $-4.5^{\mathrm{c}}$ \\
\hline III-IV & Feb. 2011 & 88 & 166 & 74 & 0 & 0 & $+3.9^{\mathrm{c}}$ \\
\hline IV-V & June 2007 & 65 & 52 & 0 & 97 & 43 & -13 to +41 \\
\hline IV-V & Feb. 2011 & 166 & 200 & 12 & 8 & 2.7 & +22 to +27 \\
\hline V-VI & June 2007 & 52 & 41 & 0 & 0 & 0 & $-11^{\mathrm{c}}$ \\
\hline V-VI & Feb. 2011 & 200 & 212 & 0 & 0 & 0 & $+12^{\mathrm{c}}$ \\
\hline
\end{tabular}

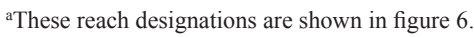

${ }^{\mathrm{b}}$ A positive value indicates net discharge of groundwater to the Verde River mainstem channel . A negative value indicates net infiltration of Verde River streamflow into the groundwater system

${ }^{\mathrm{c}}$ Because there are no diversions in this reach, there is no range for net groundwater flux using the methods of this study.

\section{Reach II-III}

Reach II-III comprises 10 mi of the Verde River that are more altered by ditch diversions than any other section of the river (fig. 6). In one section of this reach, there are three ditch diversions diverting water from the stream concurrently. Downstream of the Cottonwood Ditch diversion, streamflow decreased to less than $1.0 \mathrm{ft}^{3} / \mathrm{s}$ in June 2007 (fig. 8). The degree of human alteration to the Verde River in this reach affects all measured base-flow values in this reach.

June 2007 net groundwater flux is highly unconstrained (table 3), in that it could have ranged from -23 (net infiltration) to $+28 \mathrm{ft}^{3} / \mathrm{s}$ (net groundwater discharge to the stream) . In February 2011, the lower bound for net groundwater flux $\left(+11 \mathrm{ft}^{3} / \mathrm{s}\right.$; table 3$)$ is likely to be a reasonable value, in that it is calculated by assuming all diverted water returns to the river unconsumed. However, this lower bound would be inflated by any substantial amount of subsurface return flow of infiltrated ditch and irrigation water $\left(G W_{i n D}\right)$.

Evapotranspiration associated with field irrigation and ditch diversion in this reach ( $E T_{f}$ plus $E T_{d}$ ) would have been greater in June 2007 than in February 2011 because of the summertime growing season. Quantifying this evapotranspiration, however, is not possible with the data available in this study. Such a calculation, on the basis of fig. 5, could be possible with the following equation:

$E T_{d}+E T_{f}=\Sigma D_{\text {div }}-\Sigma D_{\text {retMeas }}-\Sigma D_{\text {retUnmeas }}-I_{d} \pm \Delta S_{d} \pm \varepsilon$.
Four terms in equation (5) are unknown: (1) unmeasured return flows, $\Sigma D_{\text {retUnmeas }}$; (2) infiltration of ditch water, $I_{d}$; (3) transient ditch-system storage changes, $\Delta S_{d}$; and (4) uncertainty, $\varepsilon$.

\section{Reach III-IV}

Reach III-IV is a comparatively short $5.6 \mathrm{mi}$ of the Verde River that spans from just downstream of an area highly modified by ditch diversions to just downstream of the confluence with Oak Creek (fig. 6). There are no ditch diversions in this reach, although they exist upstream and downstream of the reach and along Oak Creek (not shown in fig. 6). This reach has patterns of net groundwater flux similar to patterns in reach I-II, which is another reach without diversions (table 3). In June 2007, reach III-IV had a small negative net groundwater flux $\left(-4.5 \mathrm{ft}^{3} / \mathrm{s}\right.$; net infiltration), while in February 2011 it had a slightly positive net groundwater flux $(3.9 \mathrm{ft} 3 / \mathrm{s}$; net groundwater discharge to the stream). Although these values both are within 10-percent measurement uncertainty, they are consistent with the seasonal pattern of riparian evapotranspiration. The most significant aspect of this reach is the contribution of streamflow from Oak Creek (fig. 8).

\section{Reach IV-V}

Reach IV-V spans 22 mi of the Verde River and encompasses four major ditch diversions (fig. 6). One diversion (the Verde Ditch) is the longest in the Verde Valley. In June 2007, 
the Verde Ditch diverted almost the entire flow of the Verde River, reducing streamflow downstream of the diversion to less than $1.0 \mathrm{ft}^{3} / \mathrm{s}$ (fig. 8). Similar to reach II-III, this reach is considerably altered by ditch diversions.

Unlike for reach II-III, however, there is conclusive evidence that reach IV-V includes an area of substantial, focused groundwater discharge to the Verde River. Between 18 and $22 \mathrm{ft}^{3} / \mathrm{s}$ of groundwater discharged to the Verde River in a short section (about 1 river mi) near its confluence with Beaver Creek (also see the "Key Sources of Base Flow" section). This focused groundwater discharge occurred during both the June 2007 and February 2011 synoptic baseflow surveys. Sand boils observed in the streambed in this area are consistent with this considerable amount of groundwater discharge.

For many of the same reasons as for reach II-III, net groundwater flux in June 2007 was highly unconstrained (table 3); it could have ranged from -13 (net infiltration) to $+41 \mathrm{ft}^{3} / \mathrm{s}$ (net groundwater discharge). For February 2011, the lower bound for net groundwater flux was $22 \mathrm{ft}^{3} / \mathrm{s}$ (table 3 ). It probably is coincidence that this value is similar to the amount of groundwater discharging near Beaver Creek, because there also was evidence of groundwater discharging near West Clear Creek during February 2011. Overall, net groundwater flux in this reach in February 2011 likely was somewhere between 22 and $27 \mathrm{ft}^{3} / \mathrm{s}$ (table 3 ). As with reach II-III, quantifying the evapotranspiration associated with irrigated fields and ditches $\left(E T_{f}\right.$ and $\left.E T_{d}\right)$ is not possible in reach IV-V on the basis of data available in this study.

\section{Reach V-VI}

Reach V-VI spans $5.4 \mathrm{mi}$ of the Verde River, from downstream of the end of the farthest downstream ditch diversion in the Verde Valley to the Camp Verde gage (the downstream end of the study area; fig. 6). It passes through a low-population area and a landscape that becomes steep and rugged at its lower end. Little can be concluded about net groundwater flux in this reach. At best, the June 2007 and February 2011 results are consistent with the pattern seen in other reaches that lack ditch diversions (reaches I-II and III-IV): a net negative groundwater flux $\left(-11 \mathrm{ft}^{3} / \mathrm{s}\right.$; net infiltration) in June 2007 and a net positive groundwater flux $\left(+12 \mathrm{ft}^{3} / \mathrm{s}\right.$; net groundwater discharge to the stream) in February 2011 (table 3).

Although these values both are within 10-percent measurement uncertainty, they are consistent with the seasonal pattern of riparian evapotranspiration. For example, the five February 2011 discharge measurements in this reach varied between 200 and $212 \mathrm{ft}^{3} / \mathrm{s}$ (table 1), but each measurement has an uncertainty of 20 to $21 \mathrm{ft}^{3} / \mathrm{s}$ (assuming 10-percent uncertainty). Additional synoptic base-flow surveys in the future, including repeat measurements to reduce uncertainty, could be used to reach more definitive conclusions about the groundwater fluxes in reach V-VI.

\section{Summary of Reach-Level Findings}

Three reaches of the Verde River without diversions (I-II, III-IV, and V-VI) demonstrated a similar pattern in groundwater fluxes indicative of seasonal differences in riparian evapotranspiration. The pattern was one of a small net groundwater discharge to each stream reach in February 2011 $\left(12 \mathrm{ft}^{3} / \mathrm{s}\right.$ or less) and a small net infiltration of streamflow in June $2007\left(11 \mathrm{ft}^{3} / \mathrm{s}\right.$ or less $)$. Although these calculated groundwater fluxes were within the measurement uncertainty of the discharge values on which they were based and can therefore not be considered definitive, the decrease in $G W_{\text {net ND }}$ in the summer is consistent with the expected higher rates of riparian evapotranspiration $\left(E T_{r}\right)$ during that time.

Two reaches (reaches II-III and IV-V) were heavily affected by ditch diversions. These ditch systems have been studied little with regard to evapotranspiration, infiltration, or storage change, and these processes have not been monitored or estimated. While it is likely that some of the water in these reaches is consumptively used (that is, evapotranspiration from ditches and irrigated fields), presently there is not enough information available to estimate consumptive use on the basis of data in this study.

\section{Human Alterations to the Hydrologic System and Their Effects on Base Flow}

Ditches and other surface-water diversions in the Verde Valley complicate interpretation of the base-flow data contained in this and previous studies (see appendix 2). Water that is diverted from a stream is not necessarily all transpired through irrigated crops. Not all return flows were measured in synoptic base-flow surveys in this study, and even had they been measured, their flow varies considerably, and a single measurement might not be a representative or average value. There also exists a pathway by which some diverted water infiltrates the subsurface $\left(I_{d}\right)$ and flows back to the stream $\left(G W_{\text {inD }}\right)$. Quantification of these and other aspects of the hydrology of ditch diversions was beyond the scope of this report.

Although evapotranspiration rates from irrigated fields and ditch systems ( $E T_{f}$ plus $E T_{d}$ ) cannot be calculated solely on the basis of data in this study, calculations of unaccountedfor water might represent coarse estimates of the sum of $E T_{f}$ and $E T_{d}$. Unaccounted-for water is calculated with respect to a ditch diversion and is the difference between the amount of water diverted into the ditch and the sum of all measured return flows from that ditch. In June 2007, the total amount of unaccounted-for water among the seven major ditch systems in the Verde Valley was $105 \mathrm{ft}^{3} / \mathrm{s}$; in February 2011 unaccounted-for water was $37 \mathrm{ft}^{3} / \mathrm{s}$ (tables 4 and 5). Unaccountedfor water is not equal to evapotranspiration from irrigated fields and ditch systems, as there are several other pathways that unaccounted-for water may follow $\left(I_{d}, \Delta S_{d}\right.$, and $D_{\text {retUnmeas }}$ in fig. 5 and equation 5). 
A field-based survey of crop consumptive water use estimated 10,000 acre-feet of evapotranspiration from irrigated fields $\left(E T_{f}\right)$ for the 2010 growing season throughout the Verde Valley (B. Forbes, U.S. Geological Survey, written commun., 2011). Assuming a 3- to 6-month growing season, this value is equal to 28 to $55 \mathrm{ft}^{3} / \mathrm{s}$ of constant water use. This range is less than the amount of unaccounted-for water in June 2007. Because so little is known about the ditch systems, the number of possible explanations for this discrepancy is large.

Perhaps the most important fact to consider when interpreting these data is that a synoptic base-flow survey is a snapshot of a short period of time, and although helpful, it should not be over generalized. In the summer months, flow at the Camp Verde gage can vary on hourly, daily, weekly, and monthly timescales (fig. 11), reflecting many superimposed, time-lagged human and natural processes that occur upstream from this gage. This complex flow at the Camp Verde gage suggests that a synoptic base-flow survey 1 week earlier or

Table 4. Water-flow data for major active ditch diversions on the Verde River, June 20-21, 2007, Verde Valley, central Arizona.

[All units cubic feet per second; $D_{d i v}, D_{\text {retMeas }}$, and $\Sigma D_{\text {retMeas }}$ are variables defined for equations in the main body of the text associated with this table]

\begin{tabular}{|c|c|c|c|c|}
\hline \multicolumn{5}{|c|}{ June 20-21, 2007} \\
\hline $\begin{array}{c}\text { Name of } \\
\text { ditch }\end{array}$ & $\begin{array}{l}\text { Initial diversion } \\
\text { from Verde River, } \\
\text { measured or } \\
\text { calculated }^{\text {a }}\left(D_{d i v}\right)\end{array}$ & $\begin{array}{c}\text { Return flows, } \\
\text { measured } \\
\text { or estimated }{ }^{\mathrm{a}, \mathrm{b}} \\
\left(D_{\text {retMeas }}\right)\end{array}$ & $\begin{array}{c}\text { Sum of } \\
\text { measured } \\
\text { return flows } \\
\left(\sum D_{\text {retMeas }}\right)\end{array}$ & $\begin{array}{l}\text { Unaccounted } \\
\text { for diverted } \\
\text { water }^{c}\end{array}$ \\
\hline Tavasci & $8^{\mathrm{d}}$ & $5^{\mathrm{d}, \mathrm{e}, \mathrm{f}}$ & $5^{\mathrm{g}}$ & $3^{g}$ \\
\hline Hickey & 23 & $0.3 ; 1.1$ & 1.4 & 22 \\
\hline Cottonwood & $34^{\mathrm{d}, \mathrm{h}}$ & $\begin{array}{c}0.7 ; 0.8 ; 2.0 \\
2.5 ; 1.6\end{array}$ & 7.6 & 26 \\
\hline OK & 14 & $\begin{array}{c}\text { none } \\
\text { observed }\end{array}$ & 0 & 14 \\
\hline Eureka & $14^{\mathrm{d}}$ & 3.8 or $0^{\mathrm{e}, \mathrm{i}}$ & 0 to 3.8 & 10 to 14 \\
\hline Verde & $41^{\mathrm{d}}$ & $8 ; 0 ; 14^{\mathrm{d}, \mathrm{e}}$ & 22 & 19 \\
\hline Diamond S & 28 & $0.9 ; 16^{\mathrm{e}}$ & 17 & 11 \\
\hline TOTAL & $162^{a, c}$ & & $57^{\mathrm{j}}$ & $105^{\mathrm{a}, \mathrm{b}, \mathrm{j}}$ \\
\hline
\end{tabular}

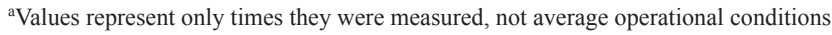
Summertime ditch operations vary on hourly, daily, weekly, and monthly time scales. Multiple entries in this column indicate multiple return-flow measurements.

${ }^{b}$ Return-flow measurements not comprehensive and ditches were not under steady-state conditions . Estimation of flows was by using the float method (Weight and Sonderegger, 2001, p. 225)

${ }^{\mathrm{c}}$ Calculated by subtraction of total measured return flows from diverted amount of water. ${ }^{\mathrm{d} I m p r e c i s e}$; calculated by subtracting two discharge measurements in Verde River.

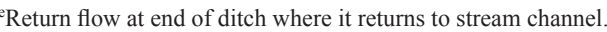

Includes some (unmeasured) amount of spring discharge.

${ }^{\mathrm{g}}$ Use of one significant figure produces this value.

hDoes not include spillback from Hickey ditch at its first siphon, which was not measured.

${ }^{i}$ Repeat visits on two days showed that this terminal return flow, which empies to Beaver Creek, was variable.

${ }^{\mathrm{j}}$ The larger measured value for Eureka Ditch return flows was used to calculate this value. later could have produced different flow measurements and different estimates of groundwater flux.

Despite all that is not yet known about Verde Valley ditches and their hydrology, recent studies and reconnaissance have led to an improved understanding of the ditches as a collection of networked and interrelated canals (fig. 12). A steady-state computer model was constructed to simulate surface-water flow in the Verde River and the four major ditches in reach IV-V (fig. 6; Ross, 2010). Recently, continuous stage-measuring equipment has been installed at key locations in some ditches (J. Haney, The Nature Conservancy, oral commun., 2011). Future studies could improve understanding of ditches through hydrologic monitoring networks and analyses designed specifically to monitor the many hydrologic components outlined in the conceptual model presented in this report (fig. 5). Because ditch operations vary hour-tohour and ditches likely are never under steady-state conditions in the summer, any such study would need to collect data

Table 5. Water-flow data for major active ditch diversions on the Verde River, February 1-3, 2011, Verde Valley, central Arizona.

[All units cubic feet per second; $D_{\text {div }}, D_{\text {retMeas }}$, and $\Sigma D_{\text {retMeas }}$ are variables defined for equations in the main body of the text associated with this table]

\begin{tabular}{|c|c|c|c|c|}
\hline \multicolumn{5}{|c|}{ February 1-3, 2011} \\
\hline $\begin{array}{c}\text { Name of } \\
\text { ditch }\end{array}$ & $\begin{array}{c}\text { Initial diversion } \\
\text { from Verde River, } \\
\text { measured or } \\
\text { calculated }^{\text {a }}\left(D_{\text {div }}\right)\end{array}$ & $\begin{array}{c}\text { Return flows, } \\
\text { measured or } \\
\text { estimated } \\
\left(D_{\text {retMeas }}\right)\end{array}$ & $\begin{array}{c}\text { Sum of } \\
\text { measured } \\
\text { return flows } \\
\left(\sum D_{\text {retMeas }}\right)\end{array}$ & $\begin{array}{c}\text { Unaccounted } \\
\text { for diverted } \\
\text { water }^{\mathrm{c}}\end{array}$ \\
\hline Tavasci & 0 & $0.9^{\mathrm{d}, \mathrm{e}}$ & 0.9 & $-1^{\mathrm{e}, \mathrm{f}}$ \\
\hline Hickey & 15 & $2 ; 0.2 ; 0.7^{\mathrm{g}}$ & 2.9 & 12 \\
\hline Cottonwood & $22^{\mathrm{g}, \mathrm{h}}$ & $0.4 ; 1.5 ; 0$ & 1.9 & 20 \\
\hline $\mathrm{OK}$ & $8^{\mathrm{g}}$ & $2.7^{\mathrm{i}}$ & 2.7 & 5 \\
\hline Eureka & 0 & $\begin{array}{c}\text { none } \\
\text { observed }\end{array}$ & 0 & 0 \\
\hline Verde & 0 & $\begin{array}{c}\text { none } \\
\text { observed }\end{array}$ & 0 & 0 \\
\hline Diamond S & 0 & $\begin{array}{c}\text { none } \\
\text { observed }\end{array}$ & 0 & 0 \\
\hline TOTAL & $45^{\mathrm{a}, \mathrm{c}}$ & & 8.4 & $37^{\mathrm{a}, \mathrm{b}}$ \\
\hline
\end{tabular}

${ }^{a}$ Values represent only times they were measured, not necessarily average operational conditions.

${ }^{b}$ Return-flow measurements not comprehensive and ditches were not under steady-state conditions . Estimation of flows was by using the float method (Weight and Sonderegger, 2001, p. 225)

${ }^{c}$ Calculated by subtraction of total measured return flows from diverted amount of water Rounding causes columns to appear to sum incorrectly.

dReturn flow at end of ditch where it returns to stream channel

eIncludes some (unmeasured) amount of spring discharge, causing an apparently negative unaccounted value.

${ }^{\mathrm{f}}$ Use of one significant figure produces this value.

${ }^{g}$ Imprecise; calculated by subtracting two discharge measurements in Verde River.

${ }^{\text {h}}$ Does not include spillback from Hickey ditch at its first siphon, which was not measured.

imprecise; inferred by subtracting an in-ditch measurement from calculated diverted amount. The location of this return flow is not known. 


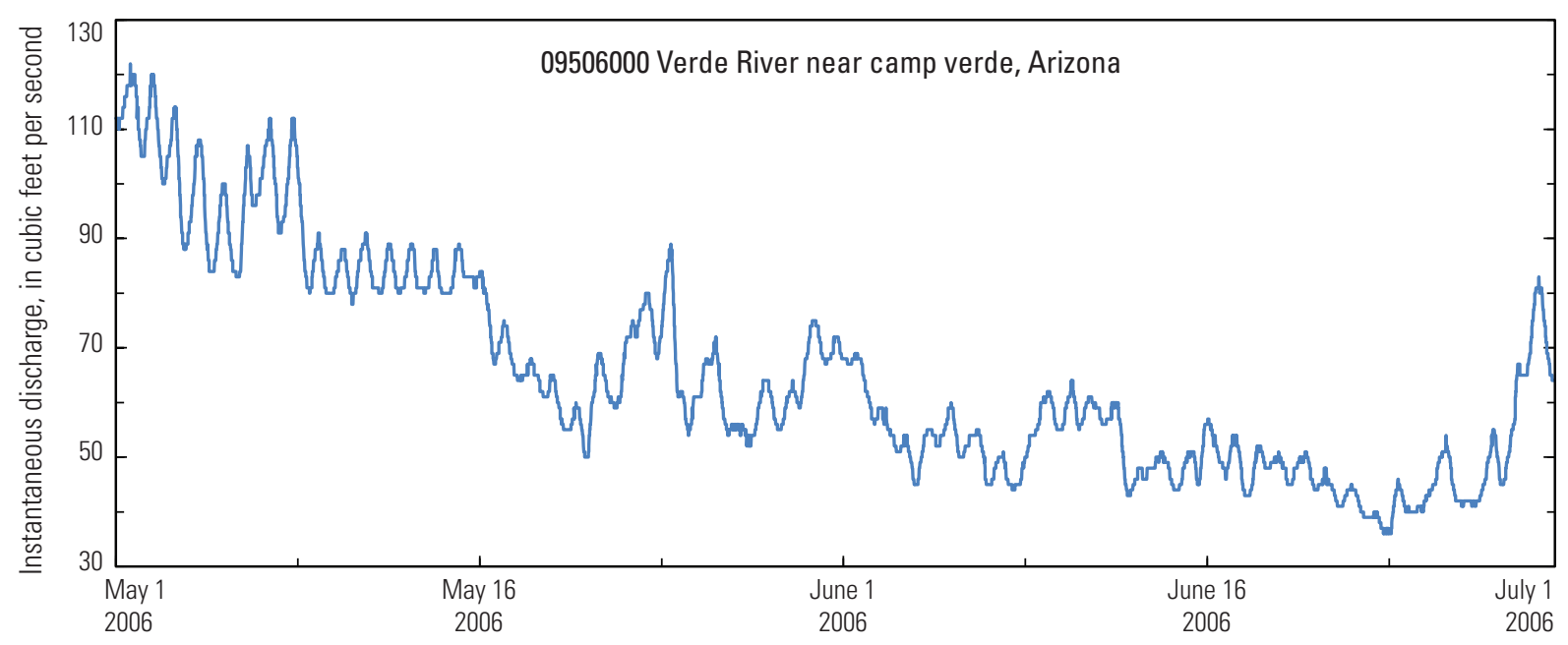

Figure 11. Instantaneous discharge at the Camp Verde gage (U.S. Geological Survey streamflow-gaging station 09506000), central Arizona, May-June 2006.

frequently to ensure a full range of variability was measured. Measurements of in-stream base flow (as in this report) alone are necessary but not sufficient for quantifying the hydrology of ditch systems and their associated irrigation.

\section{Riparian Evapotranspiration}

One natural factor that likely affected June 2007 synoptic base-flow data was riparian evapotranspiration $\left(E T_{r}\right)$. Riparian evapotranspiration includes the discharge of groundwater to plant tissues and the atmosphere rather than to a stream channel. A positive $E T_{r}$ value indicates either plants are intercepting and consuming groundwater that otherwise would have discharged to a stream, or plants are consuming stream water that infiltrated into the subsurface. Riparian evapotranspiration can be a considerable part of total groundwater discharge in arid regions and should not be ignored if total groundwater discharge is important to consider (Thomas and Pool, 2006).

Although riparian evapotranspiration is not measured directly and independently in synoptic base-flow surveys, its effects are embedded in the data collected in a synoptic base-flow survey. Active riparian evapotranspiration in a stream reach manifests as a smaller value of net groundwater flux than would have occurred had there been no vegetation in the reach.

The effects of riparian evapotranspiration in the Verde Valley-expected to be at a maximum during summer months - are superimposed on the effects of human alteration of the system, which also are at a maximum during summer months. Independent quantification of the effects of these two superimposed processes could be possible only with (1) additional measurements of water fluxes into and out of ditches, both at more locations and over longer periods of time; (2) an independent quantification of riparian evapotranspiration; or (3) simplifying assumptions about human effects or natural processes.

\section{Water Chemistry}

Specific conductance is a measure of the amount of electrical current water can transmit and is related to the ionic strength, or total amount of dissolved solids, of a water sample (Hem, 1982). In June 2007, specific conductance in the Verde River increased in the downstream direction, with values measured at the Camp Verde gage more than twice those measured at the Clarkdale gage (table 1). In February 2011, specific conductance in the Verde River did not vary much (less than 100 microsiemens per centimeter variability).

Geochemical data published by Zlatos (2008) for water samples collected during the June 2007 survey indicated that the summertime increase in ionic strength in the downstream direction could be attributed to increases in chloride, sulfate, and other ions. In June 2007, chloride concentrations increased from 13 milligrams per liter $(\mathrm{mg} / \mathrm{L})$ at the Clarkdale gage to $71 \mathrm{mg} / \mathrm{L}$ near the downstream end of Verde Valley. Sulfate concentrations increased from 7 to $204 \mathrm{mg} / \mathrm{L}$ over the same interval (Zlatos, 2008). Additional insights into the geochemical aspects of base-flow gains and losses might be possible by combining Zlatos (2008) geochemical data with data from other studies, for example Blasch and others (2006).

Measurements of $\mathrm{pH}$, water temperature, and dissolved oxygen alone were not helpful for understanding geochemical processes. The $\mathrm{pH}$ generally was near 8.0 at all measurement locations, and it varied in no discernible pattern (table 1). Water temperature and dissolved oxygen appeared to be more related to the time of day or water-flow conditions near a measuring station than to any underlying geochemical process (table 1). Future synoptic base-flow surveys likely could omit measurements of $\mathrm{pH}$, water temperature, and dissolved oxygen. Water temperature can be a useful indicator of groundwater discharge (Rosenberry and LaBaugh, 2008) if measured at a smaller spatial scale, at multiple locations in the water column, and during a time of contrasting groundwater and surface-water temperatures. 


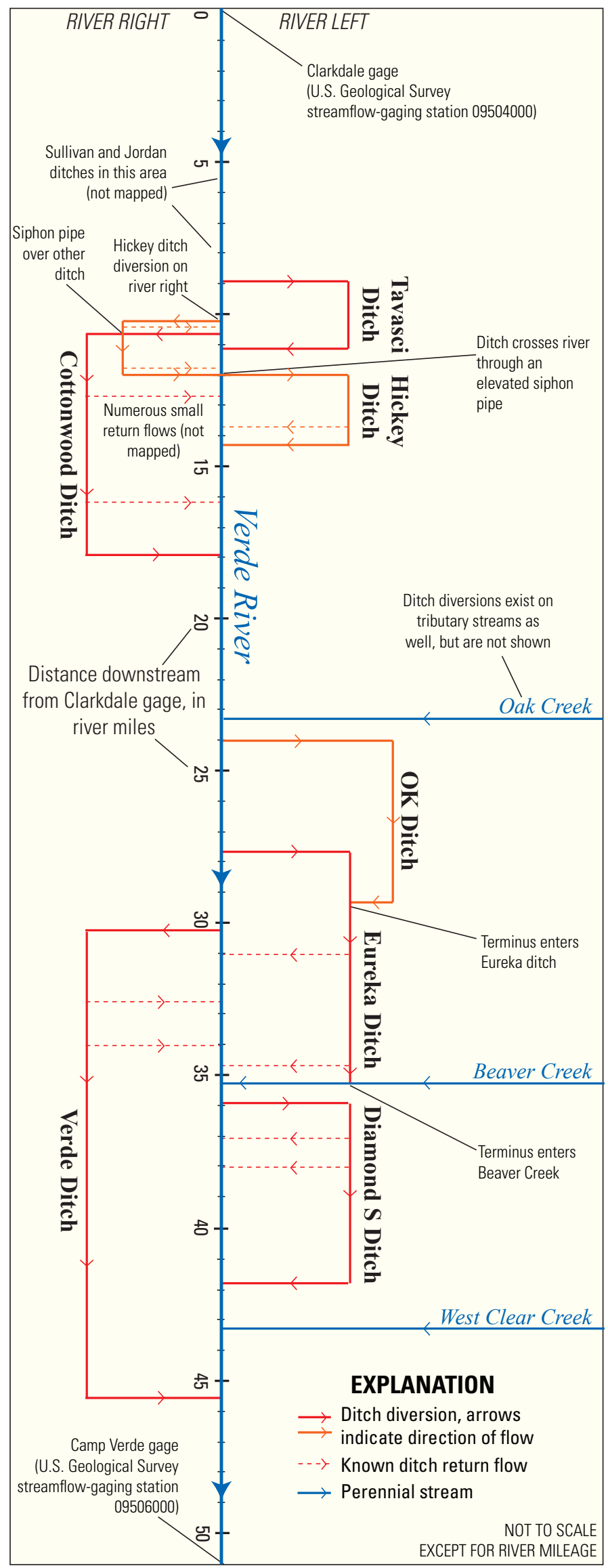

Figure 12. Schematic diagram of major irrigation ditches along the Verde River as a function of river mileage, Verde Valley, central Arizona. Return flows are indicated where known, but these have not been mapped comprehensively.

\section{Base Flow in Perennial Tributaries, June 2007}

Synoptic base-flow surveys were conducted on Oak, Beaver, and West Clear Creeks in June 2007 (fig. 6; table 6), but with a smaller measurement density than on the Verde River. Ditch diversions along these tributaries have altered the natural base-flow regime, just as they have on the Verde River mainstem. The conceptual model described in the "Conceptual Model" section and illustrated in figure 5 therefore also applies to these tributaries. However, no ditch-related measurements were made along tributaries, thus equations (2) through (4) cannot be used to interpret these data.

All three tributaries in June 2007 showed a general pattern of decreasing flow in at least some of their reaches. Beaver and West Clear Creeks decreased to zero flow near their confluence with the Verde River. Oak Creek flow decreased from a maximum of $30 \mathrm{ft}^{3} / \mathrm{s}$ to a minimum of $8.5 \mathrm{ft}^{3} / \mathrm{s}$ between river mi 33.6 and 22.7 (figs. 6 and 13); downstream of this point-ofminimum flow, flow again increased (table 6). At the confluence with the Verde River (river mi 0.1), Oak Creek discharge was about $30 \mathrm{ft}^{3} / \mathrm{s}$, less than one-half the base-flow measured at the confluence during February 2011 (table 1).

To help provide more detailed information about groundwater fluxes in these three tributaries, future studies might collect more closely spaced discharge measurements and also measure ditch diversions. Mapping and measurement of ditches along Oak Creek would be especially helpful for an improved understanding of its hydrology. Measurements of Oak Creek upstream of Sedona also might aid in understanding geologic and structural controls in this part of Oak Creek, where it enters a steep canyon and groundwater from the higher elevation Colorado Plateau is discharged.

\section{Summary}

The Verde River of central Arizona has perennial (or yearround) flow. In the absence of storm- or snowmelt-related runoff, this perennial flow is sustained by groundwater discharge- a flow component known as base flow. Base flow arises from interactions between groundwater and surface water that vary over space and time. The U.S. Geological Survey (USGS), in cooperation with Yavapai County, Arizona (in 2007) and the Verde River Basin Partnership and the Town of Clarkdale, Arizona (in 2011), conducted synoptic base-flow surveys on the Verde River in the Verde Valley. These were done to improve understanding of 
[Italicized rows indicate measurements not on tributary mainstem; UTM83, Universal Transverse Mercator 1983; $\mathrm{ft}^{3} / \mathrm{s}$, cubic feet per second; ${ }^{\circ} \mathrm{C}$, degrees Celsius; cond., conductance; $\mu \mathrm{S} / \mathrm{cm}$, microsiemens per centimeter; diss., dissolved; mg/L, milligrams per liter; $\mathrm{pH}$ in standard units; -, no measurement made; D/S, downstream; U/S, upstream]

\begin{tabular}{|c|c|c|c|c|c|c|c|c|c|c|c|}
\hline \multirow[b]{2}{*}{ Station identifier } & \multirow[b]{2}{*}{ Station name } & \multirow[b]{2}{*}{$\begin{array}{c}\text { Latitude } \\
\text { (decimal } \\
\text { degrees, } \\
\text { UTM83) }\end{array}$} & \multirow[b]{2}{*}{$\begin{array}{c}\text { Longitude } \\
\text { (decimal } \\
\text { degrees, } \\
\text { UTM83) }\end{array}$} & \multirow[b]{2}{*}{$\begin{array}{l}\text { River miles } \\
\text { upstream from } \\
\text { Verde River } \\
\text { confluence }\end{array}$} & \multicolumn{7}{|c|}{ June 26-27, 2007} \\
\hline & & & & & Date & Time & $\begin{array}{c}\text { Discharge } \\
\left(\mathrm{ft}^{3} / \mathrm{s}\right)\end{array}$ & $\begin{array}{c}\text { Water } \\
\text { temperature } \\
\left({ }^{\circ} \mathrm{C}\right)\end{array}$ & $\begin{array}{c}\text { Specific } \\
\text { cond. } \\
\text { ( } \mu \mathrm{S} / \mathrm{cm})\end{array}$ & $\begin{array}{c}\text { Diss. } \\
\text { oxygen } \\
\text { (mg/L) }\end{array}$ & $\mathrm{pH}$ \\
\hline \multicolumn{12}{|c|}{ Oak Creek } \\
\hline 09504420 & OAK CREEK NEAR SEDONA & 34.861684 & -111.761823 & 36.0 & - & - & $24^{\mathrm{a}}$ & - & - & - & - \\
\hline 345040111463600 & OAK CREEK AT CHAVEZ CROSSING SITE 11 & 34.844462 & -111.777378 & 34.3 & $6 / 27$ & $10: 50$ & 27 & 20.0 & 302 & 12.6 & 8.2 \\
\hline 345023111470700 & OAK CREEK BELOW SULLIVAN DITCH SITE 10 & 34.839740 & -111.785989 & 33.6 & $6 / 26$ & $18: 30$ & 30 & 23.1 & 300 & 6.5 & 8.5 \\
\hline 344926111480600 & OAK CREEK AT ROCK CROSSING D/S OF DITCH SITE 9 & 34.823907 & -111.802378 & 31.7 & $6 / 26$ & $16: 15$ & 14 & 26.3 & 302 & 6.8 & 8.5 \\
\hline 344905111485900 & OAK CREEK ABOVE LITTLE PARK WASH AT LOY ROAD & 34.818074 & -111.817100 & 30.5 & $6 / 26$ & $14: 30$ & 20 & 25.8 & 307 & 7.0 & 8.5 \\
\hline 344900111501500 & OAK CREEK SITE 7 & 34.816685 & -111.838211 & 27.7 & $6 / 26$ & $11: 50$ & 15 & 24.4 & 319 & 9.2 & 8.4 \\
\hline 344804111524800 & OAK CREEK BELOW ANGEL VALLEY BRIDGE SITE 6 & 34.801130 & -111.880711 & 22.7 & $6 / 26$ & $10: 27$ & 8.5 & 25.9 & 321 & 9.3 & 9.3 \\
\hline 09504500 & OAK CREEK NEAR CORNVILLE & 34.764464 & -111.890988 & 17.3 & - & - & $20^{\mathrm{a}}$ & - & - & - & - \\
\hline 344522111534700 & OAK CREEK BELOW PAGE SPRING FISH HATCHERY & 34.756131 & -111.897099 & 16.5 & $6 / 26$ & 14:03 & 21 & 23.4 & 427 & 9.2 & 7.9 \\
\hline 344441111534400 & OAK CREEK AT CROSSING ABOUT 1 MILE U/S OF SPRING CREEK & 34.744743 & -111.896266 & 14.1 & $6 / 26$ & $16: 00$ & 28 & 25.7 & 414 & 10.4 & 8.3 \\
\hline 344045111562400 & OAK CREEK AT VERDE RIVER NEAR CORNVILLE SITE 40B & 34.679189 & -111.940710 & 0.1 & $6 / 21$ & $13: 45$ & $30^{\mathrm{b}}$ & $27.3^{\mathrm{b}}$ & $445^{\mathrm{b}}$ & $8.7^{\mathrm{b}}$ & $8.4^{\mathrm{b}}$ \\
\hline \multicolumn{12}{|c|}{ Beaver Creek } \\
\hline 09505200 & WET BEAVER CREEK NEAR RIMROCK & 34.674744 & -111.672094 & 21.3 & - & - & $5.7^{\mathrm{a}}$ & - & - & - & - \\
\hline 344005111424900 & BEAVER CREEK AT CAMPGROUND D/S OF BRIDGE SITE 7 & 34.668077 & -111.714317 & 18.6 & $6 / 26$ & $17: 15$ & 5.2 & 24.2 & 269 & 9.9 & 8.2 \\
\hline 343842111461200 & BEAVER CREEK BELOW MONTEZUMA WELL SITE 5 & 34.645022 & -111.770706 & 14.5 & $6 / 26$ & $15: 17$ & 2.5 & 25.7 & 552 & 12.6 & 8.0 \\
\hline 343755111472100 & BEAVER CREEK ABOVE RUSSELL WASH SITE 4 & 34.631967 & -111.789873 & 13.0 & $6 / 26$ & $13: 40$ & 4.7 & 27.3 & 542 & 12.8 & 8.2 \\
\hline 343725111484500 & BEAVER CREEK AT RUSTY SPUR FORD SITE 3 & 34.623634 & -111.813207 & 11.4 & $6 / 26$ & $10: 54$ & 5.2 & 26.5 & 545 & 12.2 & 8.1 \\
\hline 343758111493100 & BEAVER CREEK AT FORD D/S DRY BEAVER CREEK SITE 2A & 34.632801 & -111.825985 & 9.4 & - & - & 0 & $-c$ & $-{ }^{c}$ & $-c$ & $-\mathrm{c}$ \\
\hline 343803111493900 & BEAVER CREEK BELOW DRY BEAVER CREEK SITE 2 & 34.634189 & -111.828207 & 9.2 & - & - & 0 & $-\mathrm{c}$ & $-\mathrm{c}$ & $-\mathrm{c}$ & $-\mathrm{c}$ \\
\hline 09505400 & BEAVER CREEK NEAR LAKE MONTEZUMA & 34.615000 & -111.837222 & 7.1 & $6 / 26$ & $9: 32$ & 0.4 & 22.1 & 561 & 5.7 & 8.0 \\
\hline 343502111511100 & BEAVER CREEK BELOW EUREKA DITCH FINAL RETURN FLOW & 34.583913 & -111.853763 & 0.8 & - & - & 0 & - & - & - & - \\
\hline \multicolumn{12}{|c|}{ West Clear Creek } \\
\hline 09505800 & WEST CLEAR CREEK NEAR CAMP VERDE & 34.538636 & -111.694036 & 10.6 & - & - & $13^{\mathrm{a}}$ & - & - & - & - \\
\hline 343052111452000 & WEST CLEAR CREEK ABOVE CAMPGROUND SITE 4 & 34.514498 & -111.756092 & 5.0 & $6 / 26$ & $15: 12$ & 11 & 25.9 & 350 & 8.0 & 8.4 \\
\hline 343122111481600 & WEST CLEAR CREEK LOWER DITCH SITE $2 A^{d}$ & 34.522803 & -111.805149 & - & $6 / 26$ & $12: 27$ & $8.0^{d}$ & 24.1 & 385 & 8.2 & 8.3 \\
\hline 343040111492400 & WEST CLEAR CREEK LOWER DITCH SITE $1 A^{d}$ & 34.511137 & -111.824038 & - & $6 / 26$ & $10: 28$ & $0.6^{d}$ & 22.8 & 628 & 7.2 & 8.1 \\
\hline 343040111492500 & WEST CLEAR CREEK MAIN CHANNEL ABOVE MOUTH ${ }^{\mathrm{e}}$ & 34.511220 & -111.824066 & 0.6 & - & - & 0 & - & - & - & - \\
\hline
\end{tabular}

${ }^{a}$ No direct measurement was made; this value is mean of daily-mean values published for the period of the synoptic base-flow survey.

bValue is from synoptic base-flow evaluation during preceding week; this station was not measured June 26-27, 2007.

'Not reported, as measurements were made from ponded, non-flowing water and were not considered representative.

${ }^{\mathrm{d}}$ Measurement was made in a ditch diversion, not the mainstem.

${ }^{\mathrm{e}}$ Approximate location of observation of zero flow . Only observed flow was in ditch along left bank. 
Figure 13. Synoptic base-flow measurements of Oak Creek, June 2007, Verde Valley, central Arizona.
Oak Creek Synoptic Base-Flow Survey, June 2007

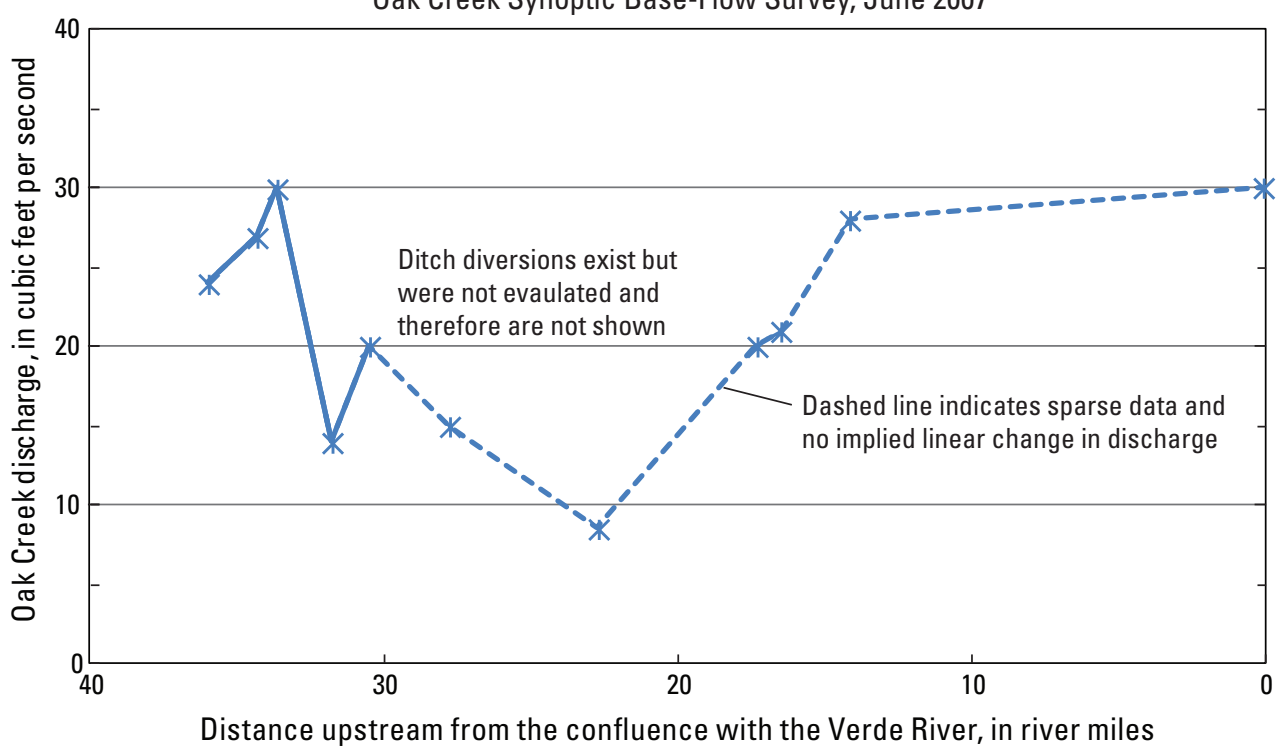

the processes affecting Verde River base flow, thereby enabling improved management of the Verde River and its connected groundwater resources. Synoptic base-flow surveys are also known as seepage runs, and are used to get a single "snapshot" of the state of a surface-water system at one moment in time.

The purpose of this report is to publish and interpret data from these synoptic base-flow surveys. The study area is the section of the Verde River between USGS streamflow-gaging stations 09504000 (Verde River near Clarkdale, Arizona; herein, the Clarkdale gage) and 09506000 (Verde River near Camp Verde, Arizona; herein, the Camp Verde gage), a distance of 51 river miles, and includes three perennial tributaries: Oak Creek, Beaver Creek, and West Clear Creek. More than 67 river diversions in the Verde Valley deliver surface water to agricultural fields and residential customers. Dozens of surface-water diversions exist in the Verde Valley; many have diverted water for over 120 years. They present a substantial and ever-present complication for understanding base flow. The ditches have not been studied comprehensively in the past or in this study. A conceptual model of a ditch-altered river system was used as a basis for discussion. Likely lower and upper bounds for net groundwater flux in five stream reaches in the Verde Valley were calculated by using equations based on this conceptual model.

Measurements in this report are called "base flow," even though humans have altered the surface-water system in the Verde Valley considerably. Measurements of base flow were made using the standard USGS methods. Streamflow entering the Verde River from tributary streams was measured in the tributary stream as close to its confluence with the Verde River as possible. Where water was observed leaving the stream into a ditch or returning to the Verde River from a ditch, attempts were made to measure that streamflow.

The Verde River presented considerably different flow regimes in June 2007 and February 2011. In February 2011, Verde River flow increased by 140 cubic feet per second $\left(\mathrm{ft}^{3} / \mathrm{s}\right)$ between the Clarkdale and Camp Verde gages. In June 2007 a reverse situation occurred, with an overall flow decrease of $23 \mathrm{ft}^{3} / \mathrm{s}$ between these gages. Surface water from the perennial tributaries of Oak Creek, Beaver Creek, and West Clear Creek was a major factor in explaining the perennial flow in the Verde River; taken on its own, this surface-water contribution explained the majority (60-63 percent) of the observed net flow increase between the Clarkdale and Camp Verde gages in February 2011. Groundwater discharge to the Verde River in the vicinity of these three tributary confluences was another important contributor of base flow to the Verde River, particularly near the confluence of Beaver Creek, where 18 to $22 \mathrm{ft}^{3} / \mathrm{s}$ of groundwater discharge was calculated based on the results of both synoptic base-flow surveys.

Five stream reaches were discussed in detail. Three of these reaches demonstrated a similar pattern of small net groundwater discharge in February $2011\left(12 \mathrm{ft}^{3} / \mathrm{s}\right.$ or less $)$ and a small net streamflow loss in June $2007\left(11 \mathrm{ft}^{3} / \mathrm{s}\right.$ or less $)$. The two other reaches were heavily affected by ditch diversions and few definitive conclusions were reached about them. Possible ranges of net groundwater flux in these reaches were calculated.

Specific conductance more than doubled from upstream to downstream in June 2007. Water temperature, dissolved oxygen, and $\mathrm{pH}$ showed only small amounts of variability that did not aid in understanding of the hydrologic system.

Synoptic base-flow surveys also were conducted in June 2007 on the tributary streams of Oak, Beaver, and West Clear Creeks. Oak Creek base flow in June 2007 decreased from a maximum of $30 \mathrm{ft}^{3} / \mathrm{s}$ to a minimum of $8.5 \mathrm{ft}^{3} / \mathrm{s}$ over a span of about 11 river miles. Beaver and West Clear Creeks decreased to zero flow near their confluence with the Verde River.

Despite all that is not yet known about the ditches and their hydrology, an improved understanding was developed through these synoptic base-flow surveys and other studies. Continued synoptic base-flow surveys in the future, along with increased hydrologic monitoring of ditches, could lead to improved understanding of base flow in the Verde Valley. Synoptic base-flow surveys, although they represent snapshots of moments in time, were found to be helpful in understanding water fluxes in the Verde Valley. 


\section{References}

Alam, J., 1997, Irrigation in the Verde Valley - a report of the irrigation diversion improvement project: Verde Natural Resource Conservation District, $96 \mathrm{p}$.

Barlow, P.M., and Leake, S.A., in press, Streamflow depletion by wells - understanding and managing the effects of groundwater pumping on streamflow: U.S. Geological Survey Circular 1376.

Blasch, K.W., Hoffmann, J.P., Graser, L.F., Bryson, J.R., and Flint, A.L., 2006, Hydrogeology of the upper and middle Verde River watersheds, central Arizona: U.S. Geological Survey Scientific Investigations Report 2005-5198, 102 p., 3 pls.

Dingman, S.L., 2002, Physical hydrology (2d ed.): New Jersey, Prentice-Hall, 646 p.

Harvey, J.W., and Wagner, B.J., 2000, Quantifying hydrologic interactions between streams and their subsurface hyporheic zones, in Jones, J.B., and Mulholland, P.J., eds., Streams and ground waters: San Diego, Academic Press, p. 3-44.

Healy, R.W., Winter, T.C., LaBaugh, J.W., and Franke, O.L., 2007, Water budgets - foundations for effective waterresources and environmental management: U.S. Geological Survey Circular 1308, 90 p.

Hem, J.D., 1982, Conductance-a collective measure of dissolved ions, in Minear, R.A., and Keith, L.H., eds., Water analysis, volume 1: New York, Academic Press, p. $137-161$.

Kennedy, J.R., and Gungle, Bruce, 2010, Quantity and sources of base flow in the San Pedro River near Tombstone, Arizona: U.S. Geological Survey Scientific Investigations Report 2010-5200, 43 p.

Konrad, C.P., Brasher, A.M.D., and May, J.T., 2008, Assessing streamflow characteristics as limiting factors on benthic invertebrate assemblages in streams across the western United States: Freshwater Biology, v. 53, no. 10, p. 1983-1998.

Leake, S.A., 2011, Capture-rates and directions of groundwater flow don't matter: Ground Water, v. 49, no. 4, p. 456-458.

Leake, S.A., and Pool, D.R., 2010, Simulated effects of groundwater pumping and artificial recharge on surfacewater resources and riparian vegetation in the Verde Valley sub-basin, Central Arizona: U.S. Geological Survey Scientific Investigations Report 2010-5147, 18 p.
Ludington, Steve, Moring, B.C., Miller, R.J., Stone, P.A., Bookstrom, A.A., Bedford, D.R., Evans, J.G., Haxel, G.A., Nutt, C.J., Flyn, K.S., and Hopkins, M.J., 2005, Preliminary integrated geologic map databases for the United StatesWestern states: California, Nevada, Arizona, Washington, Oregon, Idaho, Utah: U.S. Geological Survey Open-File Report 2005-1305 [updated 2007], accessed April 17, 2012, at http://pubs.usgs.gov/of/2005/1305/ .

National Park Service, 2012, Exploring Montezuma Well: accessed February 3, 2012, at http://www.nps.gov/moca/ planyourvisit/exploring-montezuma-well.htm .

Owen-Joyce, S.J., 1984, Hydrology of a stream-aquifer system in the Camp Verde area, Yavapai County, Arizona: Arizona Department of Water Resources Bulletin 3, 60 p.

Owen-Joyce, S.J., and Bell, C.K., 1983, Appraisal of water resources in the upper Verde River area, Yavapai and Coconino counties, Arizona: Arizona Department of Water Resources Bulletin 2, 219 p.

Pool, D.R., Blasch, K.W., Callegary, J.B., Leake, S.A., and Graser, L.F., 2011, Regional groundwater-flow model of the Redwall-Muav, Coconino, and alluvial basin aquifer systems of northern and central Arizona: U.S. Geological Survey Scientific Investigations Report 2010-5180, v. 1.1, 101 p.

Rosenberry, D.O., and LaBaugh, J.W., 2008, Field techniques for estimating water fluxes between surface water and ground water: U.S. Geological Survey Techniques and Methods book 4, chap D2, 128 p.

Ross, R.P., 2010, One-dimensional hydraulic model of Verde River near Camp Verde, Arizona, including irrigation ditch discharge: Northern Arizona University, Masters thesis, 149 p.

Sauer, V.B., and Meyer, R.W., 1992, Determination of error in individual discharge measurements: U.S. Geological Survey Open-File Report 92-144, 21 p.

Theis, C.V., 1940, The source of water derived from wells: Civil Engineering, v. 10, p. 277-280.

Thomas, B.E., and Pool, D.R., 2006, Trends in streamflow of the San Pedro River, southeastern Arizona, and regional trends in precipitation and streamflow in southeastern Arizona and southwestern New Mexico: U.S. Geological Survey Professional Paper 1712, $79 \mathrm{p}$.

Turnipseed, D.P., and Sauer, V.B., 2010, Discharge measurements at gaging stations: U.S. Geological Survey Techniques and Methods book 3, chap. A8, 87 p. (available at http://pubs.usgs.gov/tm/tm3-a8/.)

Twenter, F.R., and Metzger, D.G., 1963, Geology and groundwater in Verde Valley - the Mogollon Rim region, Arizona: U.S. Geological Survey Bulletin 1177, 132 p., 1 pl. 
Upper San Pedro Partnership, 2007, Water management of the regional aquifer in the Sierra Vista subwatershed, Arizona-2007 report to Congress: 77 p. (available at http:// usppartnership.com/docs/Sec3212007Rep011309.pdf.)

Webb, R.H., Leake, S.A., and Turner, R.M., 2007, The ribbon of green - change in riparian vegetation in the southwestern United States: Tucson, Arizona, The University of Arizona Press, $480 \mathrm{p}$.

Weight, W.D., and Sonderegger, J.L., 2001, Manual of applied field hydrogeology: New York, McGraw-Hill, 608 p.

Wilde, F.D., ed., variously dated, National field manual for the collection of water-quality data-Field measurements: U.S. Geological Survey Techniques of Water-Resources Investigations, book 9, chap. A6, sections 6.0-6.8, accessed January 27, 2012, at http://pubs.water.usgs.gov/twri9A6/.

Zlatos, C.M., 2008, Using geochemical tracers to determine aquifer connectivity, flow paths, and base-flow sources, middle Verde River watershed, central Arizona: University of Arizona, Masters thesis, $119 \mathrm{p}$. 


\section{Appendix 1. Derivation of Equations Used in Report}

The equations used in this report all are derived from the basic water-balance conservation-of-mass accounting equation (Healy and others, 2007):

$$
\text { sInflows }-\Sigma \text { Outflows }=\Delta S,
$$

where

Inflows is the sum of all water flowing into a defined control volume,

इOutflows is the sum of all water flowing out of the control volume, and

$\Delta S$ is change in storage in the control volume over some time period.

A synoptic base-flow survey can be used to calculate net rates of groundwater flux in a stream reach; gross rates cannot be inferred from its data. Because of this limitation, and for simplicity in subsequent equations, it is convenient to state:

where

$$
G W_{\text {net }}=G W_{\text {in }}-G W_{\text {out }} \text {, }
$$

$G W_{\text {net }}$ is net groundwater flux, a positive value indicating net discharge of groundwater and a negative value indicating net infiltration of streamflow into the subsurface,

$G W_{\text {in }}$ is discharge of groundwater to the stream, and $G W_{\text {out }}$ is infiltration of streamflow into the subsurface.

\section{Mainstem Stream Net Groundwater Flux, Simplified Conceptual Model}

For a conceptual model of a surface-water system involving no human alteration (see fig. 4 in main part of text), open-water evaporation assumed to be negligible, and the control volume defined as the mainstem channel of the stream reach under consideration:

where

$$
\Sigma \text { Inflows }=Q_{\text {in }}+\Sigma Q_{\text {trib }}+G W_{\text {in }} \pm \varepsilon,
$$

$Q_{\text {in }}$ is streamflow measured at the upstream end of the mainstem channel,

$\Sigma Q_{\text {trib }}$ is the sum of all measured streamflow from tributary streams that join with the mainstem channel,

$\varepsilon$ is error caused by measurement uncertainty, non-ideal measuring conditions, and deviation from stated assumptions; and: where

$$
\Sigma \text { Outflows }=Q_{\text {out }}+G W_{\text {out }} \pm \varepsilon,
$$

$$
\begin{aligned}
& Q_{\text {out }} \text { is streamflow measured at the downstream } \\
& \text { end of the mainstem channel. }
\end{aligned}
$$

Substituting equations (2), (3), and (4) into equation (1), assuming steady-state conditions $(\Delta S=0)$, and combining the error terms according to $\varepsilon \pm \varepsilon=\varepsilon$, produces:

$$
\begin{aligned}
& \left(Q_{\text {in }}+\Sigma Q_{\text {trib }}+G W_{\text {in }} \pm \varepsilon\right)-\left(Q_{\text {out }}+G W_{\text {out }} \pm \varepsilon\right)=0 \\
& Q_{\text {in }}+\Sigma Q_{\text {trib }}+G W_{\text {in }}-Q_{\text {out }}-G W_{\text {out }} \pm \varepsilon=0 \\
& G W_{\text {net }}=Q_{\text {out }}-Q_{\text {in }}-\Sigma Q_{\text {trib }} \pm \varepsilon .
\end{aligned}
$$

In a natural system that is consistent with this conceptual model and stated assumptions, a synoptic base-flow survey can measure $Q_{o u t}, Q_{\text {in }}$, and $\Sigma \mathrm{Q}_{\text {trib }}$. This means that, within an amount of error or uncertainty, $G W_{\text {net }}$ can be calculated for a stream reach using equation (5).

\section{Mainstem Stream Net Groundwater Flux, Conceptual Model Incorporating Ditches}

For a more complex conceptual model incorporating ditch diversions and return flows (see fig. 5 in main part of text), equations must reflect the fact that that ditch diversions alter groundwater and surface-water hydrology. Some water conveyed through ditches eventually infiltrates into the subsurface, reaches the water table, and after some time re-emerges as groundwater discharge to the stream channel:

$$
\begin{gathered}
G W_{i n}=G W_{i n N D}+G W_{i n D}, \\
G W_{\text {net ND }}=G W_{i n N D}-G W_{\text {out }},
\end{gathered}
$$

where

$$
\begin{gathered}
G W_{\text {inND }} \begin{array}{l}
\text { is groundwater that discharges to the mainstem } \\
\text { channel except for that which discharges } \\
\text { only because ditch diversions have altered the } \\
\text { hydrologic system, }
\end{array} \\
G W_{\text {inD }} \text { is additional groundwater that discharges to } \\
\text { the mainstem channel solely because of ditch- } \\
\text { diversion systems, and } \\
G W_{\text {netND }} \text { is net groundwater flux excluding any } \\
\text { groundwater discharge caused by ditch } \\
\text { diversions. }
\end{gathered}
$$

The next two equations describe inflows and outflows, including separation of groundwater discharge into two components per equation (6): 


$$
\begin{gathered}
\Sigma \text { Inflows }=Q_{\text {in }}+\Sigma Q_{\text {trib }}+G W_{i n N D}+G W_{i n D}+\Sigma D_{\text {ret }} \pm \varepsilon \\
\Sigma \text { Outflows }=Q_{\text {out }}+G W_{\text {out }}+\Sigma D_{\text {div }} \pm \varepsilon
\end{gathered}
$$

where

$\Sigma D_{\text {ret }}$ is the sum of all return flows from ditchdiversion systems, which includes unused water as well as excess water that runs off irrigated fields and flows back to the stream channel, and

$\Sigma D_{d i v}$ is the sum of the amount of water diverted from the stream into ditch-diversion systems.

Because of practical and time limitations, a synoptic base-flow survey typically never measures all return flows. That is:

$$
\Sigma D_{\text {ret }}=\Sigma D_{\text {retMeas }}+\Sigma D_{\text {retUnmeas }}
$$

where

$\Sigma D_{\text {retMeas }}$ is the sum of all return flows measured in a synoptic base-flow survey, and

$\Sigma D_{\text {retUnmeas }}$ is unmeasured return flows.

Substituting equations (2), (7), (8), (9), and (10) into equation (1), assuming steady-state conditions $(\Delta S=0)$ in both the stream channel and ditch system, and combining the error terms according to $\varepsilon \pm \varepsilon=\varepsilon$, produces:

$$
\begin{gathered}
\left(Q_{\text {in }}+\Sigma Q_{\text {trib }}+G W_{\text {inND }}+G W_{\text {inD }}+\Sigma D_{\text {retMeas }}+\right. \\
\left.\Sigma D_{\text {retUnmeas }} \pm \varepsilon\right)-\left(Q_{\text {out }}+G W_{\text {out }}+\Sigma D_{\text {div }} \pm \varepsilon\right)=0 \\
Q_{\text {in }}+\Sigma Q_{\text {trib }}+\mathrm{G} W_{\text {inND }}+\mathrm{G} W_{\text {inD }}+\Sigma D_{\text {retMeas }}+ \\
\Sigma D_{\text {retUnmeas }}-Q_{\text {out }}-\mathrm{G} W_{\text {out }}-\Sigma D_{\text {div }} \pm \varepsilon=0 \\
\mathrm{GW}_{\text {net } N D}=\mathrm{Q}_{\text {out }}+\Sigma \mathrm{D}_{\text {div }}-\mathrm{Q}_{\text {in }}-\Sigma \mathrm{Q}_{\text {trib }}-\Sigma D_{\text {retMeas }}- \\
\Sigma D_{\text {retUnmeas }}-G W_{\text {inD }} \pm \varepsilon .
\end{gathered}
$$

Equation (11) would simplify to equation (5) under the following conditions:

- A negligible amount of ditch water returns to the stream through the subsurface. That is, $G W_{\text {inD }}=0$. Because $G W_{\text {net }}=G W_{\text {net } N D}+G W_{\text {inD }}$, then $G W_{\text {net } N D}=G W_{\text {net }}$.

- Unmeasured return flows are negligible $\left(\Sigma D_{\text {retUnmeas }}=0\right)$.

- All diverted water returns to the stream $\left(\Sigma D_{\text {div }}=\Sigma D_{\text {retMeas }}\right)$.

- The ditch system operates under steady-state conditions.

The above assumptions are not considered to be reasonable for the Verde Valley, which is why this more complex conceptual model is used in the main part of this report.
Observations and anecdotal information about the ditch systems in the Verde Valley strongly suggest that, at least in the summertime, they might not be operating under steadystate conditions over the short period of time of a synoptic base-flow survey. One possibility for a future study may be additional (even continuous) monitoring of ditch components in equation (11). When such values are averaged over a suitably long period, the resultant average values might approximately represent a steady-state condition.

\section{Calculation of Bounds for Net Groundwater Flux}

If a mainstem reach that encompasses one or more ditches is selected, it may be possible to calculate ranges for $G W_{\text {net }}$. First, unaccounted-for water $(U)$, which encompasses all unmeasured activities in the ditch system that can cause water to not be measured as returning to the mainstem, is calculated as:

$$
U=\Sigma D_{\text {div }}-\Sigma D_{\text {retMeas }} \text {. }
$$

Substituting equation (12) into equation (11) results in:

$$
\begin{gathered}
G W_{\text {net ND }}=Q_{\text {out }}+\left(U+\Sigma D_{\text {retMeas }}\right)-Q_{\text {in }}-\Sigma Q_{\text {trib }}- \\
\Sigma D_{\text {retMeas }}-\Sigma D_{\text {retUnmeas }}-G W_{\text {ind }} \pm \varepsilon \\
G W_{\text {net } N D}=Q_{\text {out }}-Q_{\text {in }}-\Sigma Q_{\text {trib }}+U-\Sigma D_{\text {retUnmeas }}-G W_{\text {inD }} \pm \varepsilon .
\end{gathered}
$$

A lower bound for $G W_{n e t N D}$ can be calculated if all unaccounted-for water is assumed to return to the stream, unconsumed, through unmeasured surface-water return flows $\left(U=\Sigma D_{\text {retUnmeas }}\right)$. Substituting this into equation (13) produces:

$$
G W_{\text {net NDlower }}=Q_{\text {out }}-Q_{\text {in }}-\Sigma Q_{\text {trib }}-G W_{\text {inD }} \pm \varepsilon .
$$

An upper bound for $G W_{\text {netND }}$ can be calculated if all unaccounted-for water is assumed to have been returned to the atmosphere through evapotranspiration or otherwise permanently removed from the system $\left(\Sigma D_{\text {retUnmeas }}=0\right)$. Substituting this into equation (13), and resubstituting equation (12) into equation (13) produces:

$$
\begin{gathered}
G W_{\text {netNDupper }}=Q_{\text {out }}-Q_{\text {in }}-\Sigma Q_{\text {trib }}+U-G W_{\text {inD }} \pm \varepsilon \\
G W_{\text {netNDupper }}=Q_{\text {out }}-Q_{\text {in }}-\Sigma Q_{\text {trib }}+\Sigma D_{\text {div }}- \\
\Sigma D_{\text {retMeas }}-G W_{\text {inD }} \pm \varepsilon .
\end{gathered}
$$

The quantity $G W_{i n D}$ is unknown and cannot be calculated by using the synoptic base-flow data in this report. For the purposes of reporting $G W_{\text {netLower }}$ and $G W_{\text {netUpper }}$ in this report, and because there is no other obvious alternative, $G W_{i n D}$ is assumed to be negligible $\left(G W_{i n D}=0\right)$. Substituting this into equations (14) and (15) produces:

$$
\begin{gathered}
G W_{\text {net NDlower }}=Q_{\text {out }}-Q_{\text {in }}-\Sigma Q_{\text {trib }} \pm \varepsilon, \\
G W_{\text {netNDupper }}=Q_{\text {out }}-Q_{\text {in }}-\Sigma Q_{\text {trib }}+\Sigma D_{\text {div }}-\Sigma D_{\text {retMeas }} \pm \varepsilon .
\end{gathered}
$$


To review, the following assumptions underpin equations (16) and (17):

- The conceptual model of figure 5 is in effect.

- Steady-state base-flow conditions exist in the mainstem channel.

- Steady-state base-flow conditions exist in the ditch system.

- Open-water evaporation is negligible.

- No subsurface ditch return flow is occurring to the mainstem channel.

If $G W_{i n D}$ is someday determined to be substantially greater than zero, then values of $G W_{\text {netNDlower }}$ and $G W_{\text {netNDupper }}$ in this report will be too large. Therefore, the true value of $G W_{\text {netND }}$ is not necessarily bracketed by the values of $G W_{\text {netNDlower }}$ and $G W_{\text {netNDupper }}$ published in this report.

\section{Calculation of Diverted-Water Flux When Direct Measurement of a Ditch Diversion is not Possible}

Not all ditch diversions $\left(D_{\text {div }}\right)$ can be directly measured. However, $D_{d i v}$ can be calculated by rearranging equation (11), by measuring streamflow directly upstream $\left(Q_{i n}\right)$ and downstream $\left(Q_{\text {out }}\right)$ of the ditch diversion, and by making the following assumptions:

- There is no net groundwater flux between the stream channel and the subsurface $\left(G W_{\text {net ND }}=0\right)$, including groundwater discharge to the stream caused by the ditch system itself $\left(G W_{i n D}=0\right)$.

- There are no surface-water tributary inflows between the two measuring points $\left(\Sigma Q_{t r i b}=0\right)$.

- There are no ditch-diversion return flows between the two measuring points $\left(\Sigma D_{\text {retMeas }}=0, \Sigma D_{\text {retUnmeas }}=0\right)$.

- There is only one ditch diversion between the two measuring points $\left(\Sigma D_{d i v}=D_{d i v}\right)$.

Applying these assumptions to equation (11) produces:

$$
D_{\text {div }}=Q_{\text {in }}-Q_{\text {out }} \pm \varepsilon .
$$

\section{Appendix 2. Compilation of Previously Published Synoptic Base-Flow Data}

Synoptic base-flow surveys were conducted by the USGS in 1977, 1979, 1980, and 1981 and were published by Owen-Joyce and Bell (1983) and OwenJoyce (1984). These data values were compiled during this study (table 2.1). Any comparisons between these data and the 2007 and 2011 data from the present report should be undertaken carefully. Climatic conditionsand therefore rates of natural groundwater rechargelikely were different in the late 1970s than in 2007 and 2011. Ditches were in operation in the late 1970s, but the amounts of water they diverted and had consumptively used were not necessarily the same then as in 2007 and 2011. Groundwater withdrawals by pumping have been ongoing during the years intervening the 1970s and 2007, and those withdrawals could have affected base flow through streamflow capture. The effects of these processes are all superimposed. 
Table 2.1. Synoptic base-flow survey measurements for Verde River mainstem and confluences of tributaries, 1977-1981, Verde Valley, central Arizona.

[Italicized rows indicate measurements not on Verde River mainstem; multiple values indicate repeat measurements for quality assurance; UTM83, Universal Transverse Mercator 1983; ft³, cubic feet per second; cond., conductance; $\mu \mathrm{S} / \mathrm{cm}$, microsiemens per centimeter; -, no measurement made; $\mathrm{R}$, river]

\begin{tabular}{|c|c|c|c|c|c|c|c|c|c|c|c|c|}
\hline \multirow[b]{2}{*}{ Station identifier } & \multirow[b]{2}{*}{ Station name } & \multirow{2}{*}{$\begin{array}{l}\text { Latitude } \\
\text { (decimal } \\
\text { degrees, } \\
\text { UTM83) }\end{array}$} & \multirow{2}{*}{$\begin{array}{c}\text { Longitude } \\
\text { (decimal } \\
\text { degrees, } \\
\text { UTM83) }\end{array}$} & \multirow{2}{*}{$\begin{array}{c}\text { River miles } \\
\text { downstream } \\
\text { from station } \\
09504000\end{array}$} & \multicolumn{2}{|c|}{ June 20-22, 1977 } & \multicolumn{2}{|c|}{ June $11-13,1979^{\mathrm{a}}$} & \multicolumn{2}{|c|}{ November 4-7, 1980 } & \multicolumn{2}{|c|}{ June 8-11, 1981 ${ }^{b}$} \\
\hline & & & & & $\begin{array}{c}\text { Discharge } \\
\left(\mathrm{ft}^{3} / \mathrm{s}\right)\end{array}$ & $\begin{array}{c}\text { Specific } \\
\text { cond. } \\
(\mu \mathrm{S} / \mathrm{cm})\end{array}$ & $\begin{array}{c}\text { Discharge } \\
\left(\mathrm{ft}^{3} / \mathbf{s}\right)\end{array}$ & 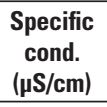 & $\begin{array}{c}\text { Discharge } \\
\left(\mathrm{ft}^{3} / \mathrm{s}\right)\end{array}$ & 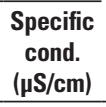 & $\begin{array}{c}\text { Discharge } \\
\left(\mathrm{ft}^{3} / \mathrm{s}\right)\end{array}$ & $\begin{array}{c}\text { Specific } \\
\text { cond. } \\
\text { ( } \mu \mathrm{S} / \mathrm{cm})\end{array}$ \\
\hline $09504000^{\mathrm{c}, \mathrm{d}}$ & VERDE R NEAR CLARKDALE & 34.852242 & -112.065994 & 0.0 & 73 & 490 & 80 & 510 & - & - & - & - \\
\hline 344618112023700 & VERDE R AT OLD BRIDGE SITE AT CLARKDALE & 34.771687 & -112.044325 & 9.6 & - & - & 74 & 599 & - & - & - & - \\
\hline 344557112014600 & VERDE R AT TUZIGOOT BRIDGE NEAR CLARKDALE & 34.765854 & -112.030158 & 10.0 & 46 & 520 & - & - & - & - & - & - \\
\hline 344557112011600 & TAVASCI MARSH WASH AT MOUTH NEAR CLARKDALE & 34.765854 & -112.021824 & - & - & - & 2.5 & 608 & - & - & - & - \\
\hline $344318111592400^{c}$ & VERDE R AT HIGHWAY 89A NEAR COTTONWOOD & 34.720299 & -111.990545 & 16.6 & 32 & 560 & - & - & - & - & - & - \\
\hline $344228111584300^{\mathrm{c}, \mathrm{d}}$ & VERDE R BELOW END OF COTTNWOOD DITCH & 34.708077 & -111.979323 & 17.8 & - & - & 67 & 655 & - & - & - & - \\
\hline 09504200 & VERDE R NEAR CORNVILLE & 34.682800 & -111.958489 & 21.5 & 43 & 600 & - & - & - & - & - & - \\
\hline 344041111571000 & VERDE R 1.3 MILES ABOVE OAK CREEK NEAR CORNVILLE & 34.678078 & -111.953488 & 21.9 & - & - & 79 & 550 & - & - & - & - \\
\hline 344052111561200 & OAK CREEK ABOVE VERDE R NEAR CORNVILLE & 34.681133 & -111.937377 & - & 33 & 430 & - & - & - & - & - & - \\
\hline $343843111555500^{\mathrm{c}, \mathrm{d}}$ & VERDE R BELOW OK DITCH TURNOUT NEAR CORNVILLE & 34.645301 & -111.932654 & 25.6 & - & - & 95 & 545 & - & - & - & - \\
\hline 343753111534600 & VERDE R BELOW HEAD OF EUREKA DITCH NEAR CAMP VERDE & 34.631412 & -111.896820 & 28.2 & - & - & 70 & 542 & - & - & - & - \\
\hline $343513111524600^{\mathrm{c}, \mathrm{d}}$ & VERDE R AT I-17 BRID GE NEAR CAMP VERDE & 34.586969 & -111.880152 & 33.3 & 13 & 620 & - & - & - & - & - & - \\
\hline $343424111513300^{c}$ & VERDE R ABOVE BEAVER CREEK NEAR CAMP VERDE & 34.573358 & -111.859874 & 34.9 & 27 & 680 & 34 & 650 & $\begin{array}{l}114 ; 115 \\
122 ; 109\end{array}$ & 610 & $\begin{array}{l}30.8 ; 29.2 \\
25.1 ; 29.8\end{array}$ & 730 \\
\hline 343428111511600 & BEAVER CREEK ABOVE VERDE R AT CAMP VERDE & 34.574469 & -111.855151 & - & 10 & 520 & - & - & 11 & 610 & 7.5 & 600 \\
\hline 343424111505900 & VERDE R BELOW BEAVER CREEK NEAR CAMP VERDE & 34.573358 & -111.850429 & 35.6 & - & - & - & - & 125 & 625 & 44.2 & 750 \\
\hline 343400111504000 & VERDE R ABOVE WASTEWATER POND AT CAMP VERDE & 34.566691 & -111.845151 & 36.2 & - & - & - & - & 110 & 640 & 47.3 & 755 \\
\hline 343319111505500 & VERDE R BELOW WASTEWATER POND AT CAMP VERDE & 34.555303 & -111.849318 & 37.1 & - & - & - & - & 90.4 & 625 & 20.8 & 730 \\
\hline $09505550^{\mathrm{e}}$ & VERDE R BELOW CAMP VERDE & 34.550581 & -111.851262 & 37.6 & 27 & 630 & - & - & $98.4^{\mathrm{f}}$ & - & $42.8^{\mathrm{f}}$ & - \\
\hline $343259111510500^{\mathrm{c}, \mathrm{d}}$ & VERDE R 0.25 MILES BELOW STATION 09505550 & 34.549747 & -111.852095 & 37.5 & - & - & 36 & 640 & 98.9 & 635 & 42.8 & 740 \\
\hline 343239111514400 & VERDE R 0.2 MILES ABOVE COPPER CANYON & 34.544192 & -111.862929 & 38.3 & - & - & - & - & 105 & 620 & 44.6 & 740 \\
\hline 343148111515100 & VERDE R 0.2 MILES BELOW RYAL CANYON NEAR CAMP VERDE & 34.530026 & -111.864873 & 39.3 & - & - & - & - & 122 & 702 & 52.7 & 815 \\
\hline 343139111503600 & VERDE R ABOVE ALLEN CANYON NEAR CAMP VERDE & 34.527526 & -111.844039 & 40.6 & - & - & - & - & 125 & 701 & 52.8 & 825 \\
\hline 343136111501300 & VERDE R AT FORT LINCOLN NEAR CAMP VERDE & 34.526692 & -111.837650 & 41.0 & - & - & - & - & 134 & 690 & 52.4 & 880 \\
\hline $343124111500400^{\mathrm{c}, \mathrm{d}}$ & VERDE R ABOVE DIAMOND S DITCH FINAL WASTEWAY & 34.523359 & -111.835150 & 41.3 & - & - & 60 & 850 & 157 & 725 & 56.3 & 890 \\
\hline 343056111501600 & VERDE R 0.15 MILES ABOVE SQUAW PEAK CANYON & 34.515581 & -111.838483 & 42.0 & - & - & - & - & 147 & 710 & 65.4 & 917 \\
\hline 343028111501700 & VERDE R BELOW SQUAW PEAK CANYON & 34.507804 & -111.838761 & 42.5 & - & - & - & - & 155 & 748 & 57.8 & 889 \\
\hline 343015111494300 & VERDE R ABOVE WEST CLEAR CREEK NEAR CAMP VERDE & 34.504193 & -111.829316 & 43.1 & 51 & 780 & 103 & 880 & $155 ; 143$ & 741 & 59.2 & 888 \\
\hline 343019111493900 & WEST CLEAR CREEK ABOVE VERDE R NEAR CAMP VERDE & 34.505304 & -111.828205 & - & - & - & - & - & 1.5 & 534 & - & - \\
\hline 343000111490800 & VERDE R 0.6 MILES BELOW WEST CLEAR CREEK & 34.500026 & -111.819593 & 43.8 & - & - & - & - & $160 ; 158$ & 715 & $79.9 ; 76.7$ & 924 \\
\hline 342913111490100 & VERDE R 1.6 MILES BELOW WEST CLEAR CREEK & 34.486971 & -111.817649 & 44.8 & - & - & - & - & $158^{\mathrm{f}}$ & 752 & $77^{\mathrm{f}}$ & 938 \\
\hline $342848111475700^{\mathrm{c}, \mathrm{d}}$ & VERDE R AT BEASLEY FLAT NEAR CAMP VERDE & 34.480026 & -111.799871 & 46.7 & - & - & 85 & 840 & 161 & 744 & 72 & 911 \\
\hline $342749111471100^{\mathrm{d}}$ & VERDE R ABOVE THE FALLS NEAR CAMP VERDE & 34.463638 & -111.787093 & 49.2 & - & - & 90 & 860 & - & - & 77.4 & 935 \\
\hline $09506000^{\mathrm{e}}$ & VERDE R NEAR CAMP VERDE & 34.448361 & -111.789870 & 50.9 & 53 & 870 & 92 & 856 & 163 & - & $92.8 ; 89.4$ & 930 \\
\hline
\end{tabular}

${ }^{\mathrm{a} F r o m ~ O w e n-J o y c e ~ a n d ~ B e l l ~(1983), ~ t a b l e ~} 14$

${ }^{b}$ From Owen-Joyce (1984), table 1. U.S. Geological Survey National Water Information System (NWIS) was used to deduce station numbers, as they were not specified in Owen-Joyce (1984). Specific conductance values were obtained from NWIS. ${ }^{\mathrm{c}}$ Measurement made at this station also in June 2007

${ }^{\mathrm{d}}$ Measurment made at this station also in February 2011

${ }^{\text {eStation }} 09505550$ is named similarly, but is not the same as station 09506000, which is farther downstream.

fValue not in published table, but available from NWIS. 

Menlo Park Publishing Service Center, California Manuscript approved for publication, August 8, 2012 Edited by Gail Sladek 
